\title{
Enter the Matrix
}

Citation for published version (APA):

Zonnebeld, N. (2019). Enter the Matrix: computational and mechanistical approaches to improve arteriovenous fistula maturation. [Doctoral Thesis, Maastricht University]. Maastricht University. https://doi.org/10.26481/dis.20190329nz

Document status and date:

Published: 01/01/2019

DOI:

$10.26481 /$ dis.20190329nz

Document Version:

Publisher's PDF, also known as Version of record

\section{Please check the document version of this publication:}

- A submitted manuscript is the version of the article upon submission and before peer-review. There can be important differences between the submitted version and the official published version of record.

People interested in the research are advised to contact the author for the final version of the publication, or visit the DOI to the publisher's website.

- The final author version and the galley proof are versions of the publication after peer review.

- The final published version features the final layout of the paper including the volume, issue and page numbers.

Link to publication

\footnotetext{
General rights rights.

- You may freely distribute the URL identifying the publication in the public portal. please follow below link for the End User Agreement:

www.umlib.nl/taverne-license

Take down policy

If you believe that this document breaches copyright please contact us at:

repository@maastrichtuniversity.nl

providing details and we will investigate your claim.
}

Copyright and moral rights for the publications made accessible in the public portal are retained by the authors and/or other copyright owners and it is a condition of accessing publications that users recognise and abide by the legal requirements associated with these

- Users may download and print one copy of any publication from the public portal for the purpose of private study or research.

- You may not further distribute the material or use it for any profit-making activity or commercial gain

If the publication is distributed under the terms of Article $25 \mathrm{fa}$ of the Dutch Copyright Act, indicated by the "Taverne" license above, 


\section{Enter the Matrix}

\section{Citation for published version (APA):}

Zonnebeld, N. (2019). Enter the Matrix: computational and mechanistical approaches to improve arteriovenous fistula maturation. Maastricht University. https://doi.org/10.26481/dis.20190329nz

\section{Document status and date:}

Published: 01/01/2019

DOI:

$10.26481 /$ dis.20190329nz

Document Version:

Publisher's PDF, also known as Version of record

\section{Please check the document version of this publication:}

- A submitted manuscript is the version of the article upon submission and before peer-review. There can be important differences between the submitted version and the official published version of record.

People interested in the research are advised to contact the author for the final version of the publication, or visit the DOI to the publisher's website.

- The final author version and the galley proof are versions of the publication after peer review.

- The final published version features the final layout of the paper including the volume, issue and page numbers.

Link to publication

\footnotetext{
General rights rights.

- You may freely distribute the URL identifying the publication in the public portal. please follow below link for the End User Agreement:

www.umlib.nl/taverne-license

Take down policy

If you believe that this document breaches copyright please contact us at:

repository@maastrichtuniversity.nl

providing details and we will investigate your claim.
}

Copyright and moral rights for the publications made accessible in the public portal are retained by the authors and/or other copyright owners and it is a condition of accessing publications that users recognise and abide by the legal requirements associated with these

- Users may download and print one copy of any publication from the public portal for the purpose of private study or research.

- You may not further distribute the material or use it for any profit-making activity or commercial gain

If the publication is distributed under the terms of Article 25fa of the Dutch Copyright Act, indicated by the "Taverne" license above, 


\section{ENTER THE MATRIX}

Computational and mechanistical approaches to improve arteriovenous fistula maturation

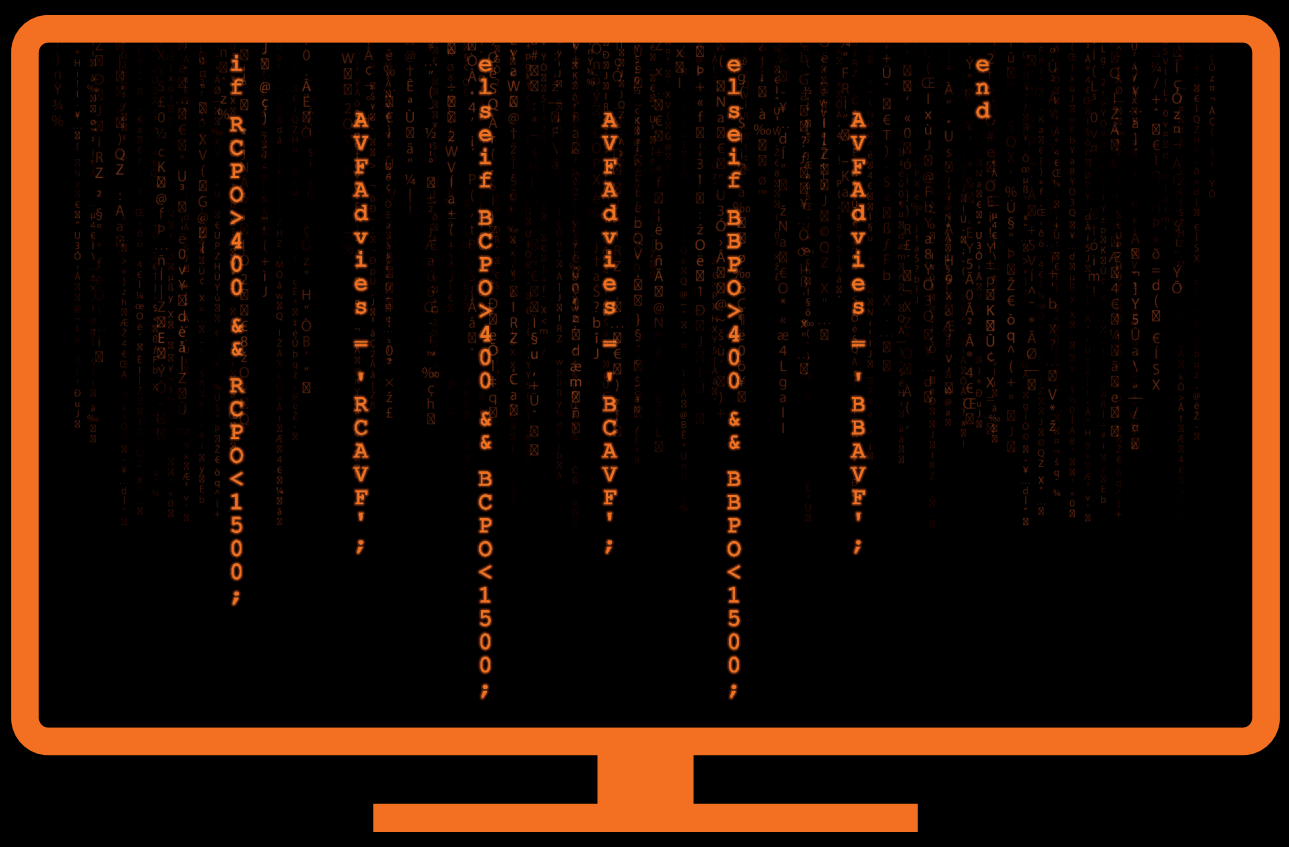

Niek Zonnebeld 


\section{ENTER THE MATRIX}

Computational and mechanistical approaches

to improve arteriovenous fistula maturation

Niek Zonnebeld 


\section{COLOFON}

Lay-out Niek Zonnebeld

Design Niek Zonnebeld

Printing Gildeprint-www.gildeprint.nl

ISBN 978-94-632-352-04

(C) 2019 Niek Zonnebeld

All rights reserved. No parts of this thesis may be reproduced, stored in a retrieval system or transmitted in any form by any means without prior permission of the author.

Supported by a grant from the Dutch Kidney Foundation (NT12.01). Financial support for publication of this thesis is gratefully acknowledged.

Additional financial support for printing of this thesis was kindly provided by: Maastricht University, Maastricht UMC+, KM Innovations B.V., Getinge, and Maatschap Heelkunde Zuid-Limburg. 


\section{ENTER THE MATRIX}

Computational and mechanistical approaches

to improve arteriovenous fistula maturation

\section{PROEFSCHRIFT}

Ter verkrijging van de graad van doctor aan de Universiteit Maastricht, op gezag van de Rector Magnificus, prof. dr. Rianne M. Letschert, volgens het besluit van het College van Decanen,

in het openbaar te verdedigen

op vrijdag 29 maart 2019 om 14:00 uur

door

Niek Zonnebeld

Geboren 3 februari 1989

Apeldoorn, Nederland 


\section{Promotores}

Prof. dr. Tammo Delhaas

Dr. Jan H.M. Tordoir

\section{Copromotor}

Dr. ir. Wouter Huberts

\section{Beoordelingscommissie}

Prof. dr. J.P. Kooman (voorzitter)

Prof. dr. N.D. Bouvy

Prof. dr. M.W. de Haan

Prof. dr. ir. P. Verdonck, Universiteit Gent, België

Dr. A.S. Bode, Catharina ziekenhuis, Eindhoven

\section{Paranimfen}

Tom Zonnebeld

Dr. Magda M. van Loon 


\section{Table of Contents}

$\begin{array}{lll}\text { Chapter } 1 & \text { General introduction } & 7\end{array}$

\section{PART I: CREATING FRAMEWORKS}

Chapter 2 Modeling strategies in arteriovenous fistula maturation and functioning: accomplishments and improvements

Chapter 3 Preoperative computer simulation for planning of vascular access surgery in hemodialysis patients

\section{PART II: EXPLORING ASSUMPTIONS}

Chapter 4 Preoperative duplex ultrasonography in arteriovenous fistula creation: intra- and interobserver agreement

Chapter 5 Natural vascular remodeling after arteriovenous fistula creation in dialysis patients with and without previous ipsilateral vascular access

\section{PART III: ASSESSING RESULTS}

Chapter 6 Preoperative patient-specific flow predictions to improve hemodialysis arteriovenous fistula maturation (Shunt Simulation Study): a randomized controlled trial

Chapter 7 Surgical and endovascular intervention for dialysis access maturation failure during and after arteriovenous fistula surgery: review of the evidence

Chapter 8 General discussion

\section{APPENDICES}

Nederlandse samenvatting

Valorization

Dankwoord

About the author 


8 ChAPTER 1 
Of the many unmet challenges in arteriovenous fistula (AVF) surgery, this thesis focuses on AVF maturation; the complex vascular remodeling process after AVF creation that eventually leads to an AVF that is usable for hemodialysis. To that end we will create frameworks, explore assumptions, and assess results. But before the reader can appreciate our work, introduction of several themes is required. First, the general clinical situation of chronic kidney disease and renal replacement therapies, in particular hemodialysis, will be described. Thereafter we will focus on the arteriovenous fistula, the preferred vascular access for hemodialysis. Not only AVF surgical techniques, but also complications after AVF creation, especially non-maturation or failure to mature (FTM), will be discussed.

Second, we will present computational modeling in general and patient-specific computational modeling in particular. The necessary steps for design and development of computational (patient-specific) models will be reviewed to properly familiarize the reader with the various aspects of the flow-predicting computational model that was used in this thesis. The chapter will end with the general aim and outline of this thesis.

\section{CHRONIC KIDNEY DISEASE AND END-STAGE RENAL DISEASE}

The kidneys serve two major functions: clearing the body of waste products, and controlling the volume and composition of body fluids. In patients suffering from chronic kidney disease (CKD), these functions deteriorate until the stage of end-stage renal disease (ESRD or CKD stage 5). Patients in this stage are in need for renal replacement therapy. Renal replacement therapy (RRT) can be offered by either kidney transplantation, peritoneal dialysis (PD) or chronic intermittent hemodialysis (HD). Both types of dialysis rely on the principle of diffusion and ultrafiltration across a semipermeable membrane. Substances in water move from areas of high concentration to areas of low concentration, e.g. potassium moves from the patient's blood toward the dialysate. A semipermeable membrane is a thin layer with pores of varying size, allowing passage of smaller solutes and fluids while simultaneously blocking passage of larger substances such as red blood cells. In peritoneal dialysis the peritoneum, i.e. the membrane lining the abdominal cavity, serves as a natural semipermeable membrane, and the dialysate is placed in the abdominal cavity through a silicone catheter. Hemodialysis on the other hand depends on an external filter, through which blood and the dialysate are pumped counter-currently to maximize the concentration gradient. Although renal transplantation is preferred over PD and HD for several reasons, it is in many patients (relatively) contraindicated (e.g. age, comorbid conditions, malignancy) or patients need to bridge the period from the start of RRT dependency until they receive a 
renal transplant. ${ }^{1}$ In these cases, the majority of patients will undergo hemodialysis. In Europe and the USA, approximately 550000 respectively 700000 patients depend on RRT, of which $60 \%$ undergo hemodialysis treatment. ${ }^{2,3}$ Nowadays, the incidence has stabilized, whereas the prevalence continues to rise. In the Netherlands, the same trends are observed, but the distribution of RRT modality differs. On a total of 17000 patients (2016), 63\% received a renal transplantation, 32\% receives $\mathrm{HD}$, and the remaining 5\% receives PD. ${ }^{4}$ This difference is mainly explained by an increasing number of pre-emptive renal transplantations in the Netherlands in comparison to other countries. Especially patients in lower age categories benefit from (pre-emptive) renal transplantation (e.g. $91 \%$ in the $0-19$ years versus $25 \%$ in the $\geq 75$ years category).

\section{Hemodialysis}

Typically, a patient receiving chronic intermittent hemodialysis is dialyzed thrice weekly for four hours per session. In order to attain efficient hemodialysis, the dialyzer needs $350-400 \mathrm{~mL} / \mathrm{min}$ of blood flow, amounting to a total volume of 84 to 96 liter that flows through the dialyzer per four hour session. As stated above, the dialyzer for HD is placed outside the body, necessitating an access to the patient's blood stream - the so-called vascular access (VA). To facilitate the tremendous volume movements, there are some specific demands for a VA. First and foremost it should be easily cannulated. To ensure a closed loop between the patients' blood stream, dialyzer, and vice-versa, two needles are used to cannulate the VA. Second, blood volume flow through the VA should be above $500 \mathrm{~mL} / \mathrm{min}$ to allow $350 \mathrm{~mL} / \mathrm{min}$ blood flow through the dialyzer on the one hand, and to prevent VA thrombosis and recirculation during hemodialysis on the other hand.

\section{Types of vascular access}

There are several types of VAs available: the central venous catheter (CVC), the prosthetic or biologic arteriovenous graft (AVG), and the arteriovenous fistula (AVF). The latter is preferred for its lower infection rates and longer patency in comparison to the other techniques. ${ }^{5,6}$ CVCs usually are inserted in the right internal jugular vein, and mainly serve as the VA of choice in acute hemodialysis. In contrast to AVGs or AVFs, a CVC can be used directly once placed. The major drawback of CVCs is the increased risk of septicemia, and therewith higher mortality rates. ${ }^{7,8}$ In AVGs the artery and the vein are connected via a prosthetic graft (usually made of polytetrafluorethylene), and this graft is used for cannulation. Although an AVG offers the benefit of earlier cannulation in comparison to AVFs (i.e. typically two vs. at least six weeks after surgical creation), the 
patency rates of AVGs are inferior to those of fistulae. In particular the primary patency of AVGs is considerably lower due to stenoses at the venous anastomosis, leading to impaired flow and subsequently thrombosis. ${ }^{9}$ Stenoses are thought to preferably develop at the venous anastomosis site of AVGs due to hemodynamical disturbances, together with a compliance mismatch between vein and graft material. ${ }^{10}$ In addition AVGs also show higher infection rates. ${ }^{9}$ The synthetically produced AVG is usually worn out by two years, and needs to be replaced. There are several graft configurations possible in the upper extremity, either in a straight (e.g. radial artery to elbow vein in the forearm, brachial artery to axillary vein in the upper arm) or looped (e.g. axillary artery to vein) fashion.

\section{Arteriovenous fistulae}

An autologous AVF is a surgically created connection between an artery and (superficial) vein. Due to the direct arteriovenous anastomosis, the large resistance in the hand is bypassed, which results in a marked decrease in peripheral resistance and thereby an increase in blood volume flow, wall shear stress and venous pressure. The vein adapts (remodels) in response to the afore-mentioned hemodynamical changes with an increase of its diameter, wall thickness, and blood volume flow. This so-called maturation is indispensable for a good functioning AVF in which the fistula is usable and safe for repetitive cannulation for hemodialysis.

Preferably, the AVF is created as distally as possible in the upper extremity in the nondominant arm. This strategy serves three purposes. Firstly, the risk of high flow complications such as hand ischemia and heart failure is reduced (see below). Secondly, using the non-dominant arm the patient will not be hindered by connecting the dialysis lines, which in turn preserves patient's independency, hand function, and mobility during dialysis. Finally, if the AVF fails, a more proximal access location may be created in the same extremity.

There are mainly three AVF locations possible. From distal to proximal these locations are: the wrist for the radiocephalic (RC) AVF (anastomosis between radial artery and cephalic vein), the elbow for the brachiocephalic (BC; anastomosis between brachial artery and cephalic vein), and the upper arm for the brachiobasilic (BB; anastomosis between brachial artery and basilic vein). After surgery the maturation process starts and by six weeks the AVF is generally considered mature when the following criteria are met: palpation of the AVF vein reveals a strong thrill and weak pulsation; upon auscultation a strong bruit with long diastolic component should be present; duplex ultrasonography (DUS) examination reveals a minimum AVF flow of $500 \mathrm{~mL} / \mathrm{min}$, and an AVF vein diameter of $4 \mathrm{~mm} .{ }^{11}$ 
Another often cited set of criteria for a mature AVF is the "Rule of $6 s$ " that requires a minimum AVF flow of $600 \mathrm{~mL} / \mathrm{min}$ in an AVF vein of minimal $6 \mathrm{~mm}$ diameter that is less than $6 \mathrm{~mm}$ deep by six weeks postoperatively. ${ }^{12}$ Although often cited, these clinical guideline criteria are more pragmatically chosen, and lack substantiation by literature in comparison to the evidence-based criteria of $500 \mathrm{~mL} / \mathrm{min}$ AVF blood flow and a $4 \mathrm{~mm}$ venous diameter.

Cumulative patency rates (i.e. the AVF remains usable for dialysis) show that the RCAVFs outperform the other two, although initially the BCAVF performs better in terms of patency rate. This discrepancy is explained by the number of RCAVF primary failures. An AVF is considered a primary failure if it thrombosed (immediately) after creation and/or cannot be revised and used for dialysis. By two years postoperatively, secondary patencies are at least $50 \%$ for the BBAVF, patency rates of RC- and BCAVFs are more or less comparable with approximately $60 \%$ at two years. ${ }^{13}$

\section{Arteriovenous fistula complications}

Complications occur in $30-50 \%$ of autologous AVFs. The complications are associated with high or low blood flow through the access, or cannulation for HD treatment. The most important complication is failure to mature (FTM) after surgical AVF creation. FTM rates are reported to be between $20-40 \%$ for all AVF types, but in particular the RCAVF is affected due to the small caliber vessels and therewith higher resistance than upper arm AVFs. ${ }^{14,15}$

Conversely, complications such as heart failure or hemodialysis access-induced distal ischemia (HAIDI) are related to high flow or low distal perfusion situations. ${ }^{16}$ Distal ischemia can ultimately lead to necrosis and/or gangrene. The AVF essentially 'over matures' - typically generating flows over $1500 \mathrm{~mL} / \mathrm{min}$ that overstrains the heart - or limits distal perfusion leading to the ischemic complaints. These complications are typically observed in BC- and BBAVFs because of the larger caliber vessels and lower resistance conduits used for AV anastomosis, and occur in approximately $20 \%$ of patients. ${ }^{17,18}$

Other AVF complications that can occur are infections, aneurysm formation, and cannulation-related complications like miscannulations and hematoma formation. Infections can either be local or superficial, or (progress to) full-blown septicemia. Underreporting of minor infections might be responsible for the paradoxical fact that septicemia is more common than minor infections, especially in AVFs. ${ }^{19}$ Aneurysms are dilatations of the AVF vein that occur in approximately $5 \%$ of AVFs, and usually develop over the course of years after AVF creation. ${ }^{20}$ Aneurysms can require treatment when for example the skin is compromised, cannulation difficulties exist, or the aneurysm causes 
pain due to nerve compression. Treatment can either be ligation of the AVF, aneurysm resection with interposition graft, or resection of excess length and luminal reduction. ${ }^{20,21}$ Miscannulation is defined as the need to insert more than one needle per arterial or venous connection, due to nonfunction of the previous needle. Hematomas are abnormally localized infiltrations due to (mis)cannulation. Not surprisingly, these types of complications occur frequently, and more often in AVFs than in AVGs. ${ }^{22}$

\section{Preoperative work-up for the creation of an arteriovenous fistula}

An extensive preoperative work-up is performed in an effort to provide the patient with a functional AVF. At physical examination the superficial veins are inspected and palpated to assess their quality and the length of a straight cannulation segment. Ideally, the future AVF has a length of $10 \mathrm{~cm}$ so cannulation can be performed at many sites to let the AVF heal in-between sessions. Furthermore, special interest is paid to any venous collateral formation around the shoulder and on the chest because many patients previously had CVCs with their inherent high risk of central venous stenoses. Since central venous outflow obstructions with arm swelling usually become symptomatic after AVF creation, it is important to preoperatively perform venograms to rule out obstruction. Furthermore, the arteries are assessed for their quality of pulsations and, using the Allen test, for the presence of collateral perfusion of the hand. Blood pressure measurements of both arms are obligatory, since a difference of $>20 \mathrm{mmHg}$ may be indicative for a proximal arterial stenosis. The skin is inspected for infection, ulcers, et cetera. $^{23,24}$

Duplex ultrasonography is performed with examination of the veins and arteries from the wrist to the shoulder. The veins are assessed for their diameters at multiple discrete locations and, more importantly, for their continuity to the central venous outflow. The arteries are examined for their diameters at discrete locations as well, and for the presence of any stenoses. The latter is important since a stenosis can become hemodynamically important when an AVF is constructed distally, resulting in malperfusion of the hand (i.e. an increased risk on HAIDI). Following the discrete diameter measurements, the most distal configuration that surpasses the cut-off values mentioned in clinical guidelines is then determined and the operative plan drawn up accordingly. Based on multiple studies that have shown that smaller caliber vessels are related to higher FTM rates, ${ }^{25,} 26$ (inter)national guidelines recommend minimal artery and vein diameters of 2 and $3 \mathrm{~mm}$ for forearm and upper arm AVFs respectively. ${ }^{27-30}$ 


\section{Clinical studies that aim to reduce failure to mature rates}

One of the most extensively studied subjects in relation to AVF maturation is the preoperative DUS examination mentioned above. Wong et al. and Malovrh were the first ones to publish their studies that related AVF maturation rates with preoperative arterial and/or venous diameters $\left(<1.6\right.$ and $\leq 1.5 \mathrm{~mm}$ respectively). ${ }^{25,}{ }^{26}$ Many other studies thereafter have confirmed this relation between preoperative vessel size in relation to maturation rates, although exact cut-off values differ. ${ }^{31-33}$ Furthermore several randomized clinical trials were conducted to assess the influence of routine (versus no or selectively used) preoperative DUS examination on AVF maturation with conflicting results. ${ }^{34-38}$ Although there is an ongoing debate regarding the routine use of preoperative DUS examination, ${ }^{39,} 40$ clinical guidelines do advocate the use of preoperative vessel mapping..$^{28}$

Preoperative vessel anatomy, however, is only one of many factors involved in the process of arteriovenous fistula maturation. It is a highly complex process with many interacting factors - from hemodynamical to inter- and intracellular. The importance of unraveling this complex interplay is well recognized. The American Hemodialysis Fistula Maturation (HFM) study for example aims to unravel underlying mechanism of maturation from four domains: anatomical (e.g. vessel diameters), biological (e.g. molecular characteristics), clinical attributes (e.g. demographics), and process of care (e.g. surgical data). ${ }^{41}$ The REDUCE-FTM study by Lok et al. aimed to develop and validate a scoring system to stratify patient's risk for FTM. ${ }^{42}$ Although promising at first, it is now clear that such an approach lacks generalizability. The Lok score is derived from multivariate regression analysis on only a few clinical patient characteristics. Masengu et al. and Lilly et al. tried but failed to validate this risk score in their hemodialysis populations in respectively Ireland and the United States..$^{15,43}$ Demographic differences in their population, in comparison to the Canadian population of Lok et al. explains the failed validation, underscoring the major limitation of epidemiological/statistical models. Furthermore, many studies, recently summarized by Viecelli et al., focus on systemic therapies to reduce FTM rates, including the use of antiplatelet agents, statins, renin-angiotensin-aldosteron-system inhibitors, and the panacea fish oil. ${ }^{44}$ Irrespective of any found effect on FTM rates of the studies described above, two important issues arise from this discussion. Firstly, the large variation in underlying hypotheses of aforementioned studies corresponds with the high complexity of AVF maturation. Secondly, even when an effect of any intervention is found, there always remains a proportion of AVFs that fail to mature. In these cases there are several options at hand to treat FTM. Treatments strategies for FTM are manifold and include both 
surgical (e.g. proximal neo-anastomosis), and endovascular (e.g. balloon assisted maturation) interventions.

\section{COMPUTATIONAL MODELING}

Although AVF maturation is a complex interplay of many factors, hemodynamical factors such as vessel geometry, and especially blood (volume) flow are consistently found to relate to maturation. The latter not only for the fact that maturation by definition requires a minimal blood volume flow, but also because many (intra)cellular processes relate to wall shear stress alterations. ${ }^{45}$ It was therefore hypothesized that a computational model that patient-specifically predicts postoperative flows could serve as a decision-support tool. Consequently, a European consortium (i.e. the ARCH project; 'patient specific image-based computational modeling for improvement of Acute and long-teRm outComes of vascular access for Hemodialysis') was formed to develop this predictive computational model. The ARCH consortium consisted of many industrial, technical, and clinical partners that have collaborated to develop said model, but also explored alternative preoperative imaging modalities.

The ARCH project produced a multitude of scientific publications. The majority focused on the development of the flow predicting model (discussed below), but also the feasibility of non-contrast enhanced magnetic resonance angiography was investigated, ${ }^{46}$ as well as the reproducibility of pulse-wave velocity assessment prior to vascular access creation. ${ }^{47}$

\section{How to design a patient-specific computational model - the medical engineering chain}

Making cardiovascular computational models suitable for decision-support is a demanding and resource consuming endeavor. To eventually ensure a translation of the computational model from a pre-clinical to clinical phase (i.e. desk-to-bedside), a structured approach is needed. The experiences during the $\mathrm{ARCH}$ project have led to a formalization of all these steps in a medical engineering (ME) modeling chain. ${ }^{48}$ This $\mathrm{ME}$ modeling chain describes the various necessary pre-clinical and clinical iterative and flexible steps. An iterative and flexible approach is indispensable because model complexity and output accuracy are unknown beforehand. Before going into detail of the various steps, it is important to realize the challenges faced whilst developing computational models. The ME modeling chain basically serves as a handle to cope with these challenges. 


\section{Modeling challenges}

First and foremost, engineers and medical doctors should be on the same line; they should focus on a relevant and meaningful model outcome metric. This might seem a platitude, but engineers and medical doctors have opposing problem solving approaches - mechanistic versus probabilistic - that are inherent to their formal training. Engineers for example are used to accurate measurements and/or calculations (e.g. forces exerted on suspension bridges), and clear output/outcomes (e.g. bridge does not collapse). Problem solving is then relatively straightforward: in order to prevent the bridge from collapsing, more or stronger suspenders are needed. Conversely, medical doctors have very few certainties, and inevitably are implicit appraisers. Input variables for their mental models are more or less clouded (e.g. vessel diameter measurements respectively patient's sex), but the same set of input does not always lead to the same outcome (e.g. AVF maturation). Therefore, the major challenge faced by engineers is to identify a relevant and important enough metric to support the complicated process of clinical decision-making. Obviously, this metric will be used in the probabilistic clinical setting, and thus can never be the only decisive factor.

The second challenge is the inevitable tradeoff between model complexity and accuracy. The more complex a model is, the closer it is to (the patient-specific) reality, but each input variable has a given degree of uncertainty that is then introduced into the model. Model error is related to the complexity of the model, and is primarily introduced by making assumptions. A model is always a simplification of the actual system. It should therefore not be too simple as it will not sufficiently capture relevant physics and physiology. A (too) complex model on the other hand is neither desirable. Practical limitations for example are the limited and costly clinical resources, patient burden when undergoing all necessary measurements, or the mere fact that certain model parameters cannot be measured at all. Measurement uncertainties, however, are even more important. Not only are measurements inherently uncertain, also the physiological envelop of the patient introduces uncertainty (e.g. constant variability of superficial vein diameters throughout the day).

\section{Pre-clinical phase: developing a patient-generic model}

In the pre-clinical phase, four iterative steps that ultimately result in a patient-generic model are to be completed. First, a model that is based on physical laws in relation to known (patho)physiological factors is designed during model development. Usually, at this point the model is still based on literature and/or expert opinion. Second, the model's capability (i.e. mathematical accuracy) to capture relevant physics is assessed 
during model verification in vitro and ex vivo experiments. Third, at mechanical characterization, the mechano-biological characteristics of for example the vascular wall are assessed in order to provide the model with relevant material behavior. Fourth, model implementation entails the possibility to already use the generic model for observing general trends in for example "virtual AVF surgery" or hypothesis generation.

\section{Clinical phase: is the developed model ready for patient-specific decision support?}

To introduce the model that completed the previous steps in clinical practice, and more specifically for patient-specific decision support, several steps should be taken in the clinical phase. First, clinical workflow analysis aims to integrate the model in routine clinical workflow. This includes that both model input preferably is attained using routing imaging modalities, and to tune model output with clinical meaningful measures (e.g. blood volume flow). In this way, conventional decision-making is minimally interfered. Second, during model optimization the model is applied to already available patient data for simulation and compared with clinical data. It is basically a validation step on retrospective data, and is therefore pre-eminently usable for iterative optimization. Model shortcomings (e.g. missing parameters) in the clinical setting are identified, while simultaneously the agreement with clinical workflow is checked. Third, model personalization will ultimately result in the measurement protocol to allow patient-specific simulations. During this step, global sensitivity analyses are used for parameter prioritization and fixation. Parameter prioritization aims to identify those parameters of major influence that should therefore be measured or assessed most accurately. Parameter fixation on the other hand aims to identify those parameters that are of minor of negligible influence, so that these can be set to constants. Fourth, now that the measurement protocol is drawn up, the accuracy of the model outcome is assessed during model corroboration. Here, the outcome metric is compared with actual clinical measurements (e.g. postoperative AVF flow). For this step, it is important to consider both the uncertainty in predictions and measurements. In case of any measure (or both) has huge uncertainty, there might be overlap or agreement between the two, but the outcome metric is then useless for decision support. In this case one should revisit one of the previous steps in the modeling chain - maybe even go back to the preclinical phase. However, if this step is completed satisfactorily, there is a proof-ofprinciple of the model's capabilities, and its clinical merits should be assessed. The final step in the modeling chain is assessment of the model's impact on clinical decision-making by means of a randomized clinical trial. As mentioned before, a computational model can never exclusively be the decisive factor. It should rather be seen as an additional 
diagnostic tool. Therefore, also diagnostic values such as sensitivity, specificity, positive and negative predictive values are assessed.

\section{Predicting postoperative arteriovenous fistula flows - the ARCH model}

The ARCH model, a so-called pulse wave propagation model (PWPM), is used to predict postoperative AVF flows. PWPMs are based on mass and momentum balance laws, and describe how pressure and flow pulses travel along the arterial tree. ${ }^{49}$ For this, the part of the arterial tree that is of interest is divided in several serially connected segments that describe the local relation between pressure and flow via physical parameters as resistance, compliance, and inertia. The parameters of each individual segment can be made patient-specific by imputation of volume flows and diameters, measured at the corresponding anatomical locations for an individual patient. These patient-specific measurements than lead to a personalized model that, in this case, predicts expected postoperative AVF flows for the various configurations. The major advantage of this approach is its patient-specific nature. Many of the previously discussed studies typically rely on population statistics, that often lack generalizability, or the outcome measure is difficult to interpret for the individual patient.

The experiences of the $\mathrm{ARCH}$ project resulted in formalizing the necessary steps described above. Consequently, all steps are taken but not necessarily in the now proposed order. First, the clinically relevant output was determined in consultation with the vascular surgery department, and clinical workflow analysis was already performed by deciding that the model should be DUS fed, as this is clinical routine. Then, the patient generic-model was designed, ${ }^{50}$ which was a literature and expert opinion guided extension on an already available and experimentally validated arterial computational model. ${ }^{51,52}$ Again, this extended AVF model was validated in vitro. ${ }^{53}$ Then, model parameter prioritization ${ }^{54}$ and fixation ${ }^{55}$ was performed using sensitivity analysis, and the measurement protocol for model corroboration was drawn up accordingly. ${ }^{56}$ Thereafter, model corroboration showed that the model was capable to relatively accurately predict direct postoperative AVF flow. ${ }^{57}$ In $70 \%$ of the cases the flow prediction overlapped with the flow measurement, and in $76 \%$ the proposed AVF configuration based on flow predictions was in agreement with an experienced vascular surgeon. The final step in the modeling chain of the ARCH model, assessment of the ARCH model's impact on clinical decision-making by means of a randomized clinical trial, is the main objective of this thesis. 


\section{GENERAL AIM AND OUTLINE OF THE THESIS}

Arteriovenous fistula surgery is thus a challenging endeavor, in part due to the incompletely understood complexity of AVF maturation. There are, however, many initiatives and innovations that aim to alleviate the encountered challenges. For AVF maturation, computational modeling is probably the most innovative approach, but still needs to complete the final step of assessing its ability to improve maturation rates.

Therefore, the general aim of this thesis is to alleviate several of the encountered challenges in AVF surgery mentioned above, in particular that of AVF maturation. The main objective is to investigate the effect of patient-specific flow predictions on maturation rates.

\section{PART I: Creating frameworks}

In Chapter 2, the computational model under investigation in this thesis is put into a broader perspective. The complexity of underlying phenomena of AVF maturation is still incompletely understood. Knowledge lacunae are partly due to the complexity per se, but more importantly because usually only a limited number of factors is studied, or the various factors are not studied in their mutual dependency. Why this observation calls for a more integrative approach, and how computational models can be of benefit, is discussed here. The complexity of underlying phenomena of AVF maturation in relation to various integrative epidemiological and computational modeling approaches will therefore be reviewed.

Chapter 3 describes the study protocol and trial design of the Shunt Simulation Study, a multicenter randomized controlled trial that aims to assess the clinical merits of the computational model - the main objective of this thesis. Patients will be randomized for conventional surgical planning or computational model aided surgical planning.

\section{PART II: Exploring assumptions}

Chapter 4 focusses on the repeatability (or intraobserver) and reproducibility (interobserver agreement) of the DUS measurements that serve as model input. DUS is the routine imaging modality in clinic, and was therefore selected for model input acquisition. Interestingly enough both aforementioned degrees of agreement are still undetermined. Although one could argue that these measures are irrelevant differences within and between observers are an invariable property of DUS and dealt with accordingly in clinical practice (i.e. probabilistic setting described above) - they are 
important measures. Clinically, there is an ongoing (international) debate whether DUS should be performed routinely in preoperative work-up. Knowledge of repeatability and reproducibility can add to this debate. From a modeling perspective, both degrees of agreement (or disagreement) introduce random errors that can influence model outcome. Quantifying the random errors can result in a better estimation of the model's uncertainty domains.

In Chapter 5 a ten-year retrospective study on natural vascular remodeling after AVF surgery is presented. Although it is clear that after AVF surgery profound vascular remodeling occurs, it remains to be elucidated if one could expect differences in these changes between patients with and without a previous ipsilateral VA. Patients with a previous ipsilateral VA might show some sort of vessel preconditioning that potentially influence AVF maturation in a positive way. Furthermore, the changes are assessed beyond the often studied six postoperative weeks to determine how long one can expect an AVF to develop. In addition, this study explores the assumption that the computational model is not designed for patients with a previous ipsilateral VA due to the altered hemodynamics.

\section{PART III: Assessing results}

Chapter 6 entails the clinical results of the Shunt Simulation Study on AVF maturation rates, and how clinical decision-making is influenced. Although the study is still ongoing, it can already be determined if and to what extent the computational model can contribute to clinical decision-making. Although AVF flow importantly influences the maturation likelihood, it is a multifactorial process. In other words: flow does not equal to maturation. Furthermore, its performance can be related to other models or approaches that aim to reduce FTM rates.

In Chapter 7 the current evidence for the various surgical and endovascular interventions for FTM is reviewed. Despite all efforts to reduce FTM rates, it probably will always be an issue that needs frequent interventions to resolve. There are numerous treatment possibilities available - both surgical and endovascular. In this chapter many of the treatment possibilities are discussed in terms of timing, and success.

To conclude, Chapter 8 provides a general discussion of the major findings of all aforementioned chapters. The relations between one and another finding will be discussed and, more importantly, the findings will be put in a broader perspective. Furthermore, future clinical and research perspectives for AVF creation will be explored. 


\section{REFERENCES}

1. Thiruchelvam PT, Willicombe $\mathrm{M}$, Hakim N, et al. Renal transplantation. Bmj. 2011;343:d7300.

2. Kramer A, Pippias M, Noordzij M, et al. The European Renal Association - European Dialysis and Transplant Association (ERA-EDTA) Registry Annual Report 2015: a summary. Clinical kidney journal. 2018;11(1):108-22.

3. System USRD. USRDS annual data report: An overview of the epidemiology of kidney disease in the United States. 2017; Volume Two: End-Stage Renal Disease in the United States.

4. Hoekstra T, Van Ittersum F, Hemmelder MH. RENINE year report 2016. 2016.

5. Pisoni RL, Arrington CJ, Albert JM, et al. Facility hemodialysis vascular access use and mortality in countries participating in DOPPS: an instrumental variable analysis. American journal of kidney diseases. 2009;53(3):475-91.

6. Ravani P, Palmer SC, Oliver MJ, et al. Associations between hemodialysis access type and clinical outcomes: a systematic review. Journal of the American Society of Nephrology. 2013;24(3):465-73.

7. Xue $\mathrm{H}, \mathrm{Ix} \mathrm{JH}$, Wang $\mathrm{W}$, et al. Hemodialysis access usage patterns in the incident dialysis year and associated catheter-related complications. American journal of kidney diseases. 2013;61(1):123-30.

8. Dhingra RK, Young EW, Hulbert-Shearon TE, et al. Type of vascular access and mortality in U.S. hemodialysis patients. Kidney international. 2001;60(4):1443-51.

9. Almasri J, Alsawas $M$, Mainou $M$, et al. Outcomes of vascular access for hemodialysis: A systematic review and meta-analysis. Journal of vascular surgery. 2016;64(1):236-43.

10. Roy-Chaudhury P, Sukhatme VP, Cheung AK. Hemodialysis vascular access dysfunction: a cellular and molecular viewpoint. Journal of the American Society of Nephrology. 2006;17(4):1112-27.

11. Robbin ML, Chamberlain NE, Lockhart ME, et al. Hemodialysis arteriovenous fistula maturity: US evaluation. Radiology. 2002;225(1):59-64.

12. National Kidney Foundation Kidney Disease Outcomes Quality Initiative: clinical practice guidelines for vascular access, update 2006. American journal of kidney diseases. 2006;48(Suppl 1):S176-247.

13. Wilmink T, Hollingworth L, Powers $S$, et al. Natural History of Common Autologous Arteriovenous Fistulae: Consequences for Planning of Dialysis Access. European Journal of Vascular and Endovascular Surgery. 2016;51(1):134-40.

14. Huijbregts $\mathrm{HJ}$, Bots $\mathrm{ML}$, Wittens $\mathrm{CH}$, et al. Hemodialysis arteriovenous fistula patency revisited: results of a prospective, multicenter initiative. Clinical journal of the American Society of Nephrology. 2008;3(3):714-9.

15. Masengu A, Maxwell AP, Hanko JB. Investigating clinical predictors of arteriovenous fistula functional patency in a European cohort. Clinical kidney journal. 2016;9(1):142-7. 
16. Tordoir JH, Dammers R, van der Sande FM. Upper extremity ischemia and hemodialysis vascular access. European journal of vascular and endovascular surgery. 2004;27(1):1-5.

17. Basile $C$, Lomonte $C$, Vernaglione $L$, et al. The relationship between the flow of arteriovenous fistula and cardiac output in haemodialysis patients. Nephrology, dialysis, transplantation. 2008;23(1):282-7.

18. Scheltinga MR, van Hoek F, Bruijninckx CMA. Time of onset in haemodialysis accessinduced distal ischaemia (HAIDI) is related to the access type. Nephrology Dialysis Transplantation. 2009;24(10):3198-204.

19. Lok CE, Foley R. Vascular access morbidity and mortality: trends of the last decade. Clinical journal of the American Society of Nephrology. 2013;8(7):1213-9.

20. Pasklinsky G, Meisner RJ, Labropoulos N, et al. Management of true aneurysms of hemodialysis access fistulas. Journal of vascular surgery. 2011;53(5):1291-7.

21. Woo K, Cook PR, Garg J, et al. Midterm results of a novel technique to salvage autogenous dialysis access in aneurysmal arteriovenous fistulas. Journal of vascular surgery. 2010;51(4):921-5, 5 e1.

22. van Loon MM, Kessels AG, Van der Sande FM, et al. Cannulation and vascular access-related complications in hemodialysis: factors determining successful cannulation. Hemodialysis international International Symposium on Home Hemodialysis. 2009;13(4):498-504.

23. Beathard GA, editor Physical examination of the dialysis vascular access. Seminars in dialysis; 1998: Wiley Online Library.

24. Sousa CN, Apostolo JL, Figueiredo $\mathrm{MH}$, et al. Physical examination: how to examine the arm with arteriovenous fistula. Hemodialysis international International Symposium on Home Hemodialysis. 2013;17(2):300-6.

25. Wong V, Ward R, Taylor J, et al. Factors associated with early failure of arteriovenous fistulae for haemodialysis access. European journal of vascular and endovascular surgery. 1996;12(2):207-13.

26. Malovrh M. Non-invasive evaluation of vessels by duplex sonography prior to construction of arteriovenous fistulas for haemodialysis. Nephrology, dialysis, transplantation. 1998;13(1):125-9.

27. Sidawy AN, Spergel LM, Besarab A, et al. The Society for Vascular Surgery: clinical practice guidelines for the surgical placement and maintenance of arteriovenous hemodialysis access. Journal of vascular surgery. 2008;48(5 Suppl):2S-25S.

28. Schmidli J, Widmer MK, Basile C, et al. Vascular Access: 2018 Clinical Practice Guidelines of the European Society for Vascular Surgery (ESVS). European Journal of Vascular and Endovascular Surgery. 2018;55(6):757-818.

29. Tordoir J, Canaud B, Haage P, et al. EBPG on Vascular Access. Nephrology, dialysis, transplantation. 2007;22 Suppl 2:ii88-117.

30. NVVH. Richtlijn Shuntchirurgie. 2010.

31. Allon M, Lockhart ME, Lilly RZ, et al. Effect of preoperative sonographic mapping on vascular access outcomes in hemodialysis patients. Kidney international. 2001;60(5):2013-20. 
32. Tordoir JH, Rooyens P, Dammers R, et al. Prospective evaluation of failure modes in autogenous radiocephalic wrist access for haemodialysis. Nephrology, dialysis, transplantation. 2003;18(2):378-83.

33. Silva MB, Jr., Hobson RW, 2nd, Pappas PJ, et al. A strategy for increasing use of autogenous hemodialysis access procedures: impact of preoperative noninvasive evaluation. Journal of vascular surgery. 1998;27(2):302-7; discussion 7-8.

34. Mihmanli I, Besirli K, Kurugoglu S, et al. Cephalic vein and hemodialysis fistula: surgeon's observation versus color Doppler ultrasonographic findings. Journal of ultrasound in medicine. 2001;20(3):217-22.

35. Nursal TZ, Oguzkurt L, Tercan F, et al. Is routine preoperative ultrasonographic mapping for arteriovenous fistula creation necessary in patients with favorable physical examination findings? Results of a randomized controlled trial. World journal of surgery. 2006;30(6):1100-7.

36. Zhang Z, Wang X, Zhang Z, et al. Hemodynamic evaluation of native arteriovenous fistulas for chronic hemodialysis with color Doppler ultrasound. Chin J Med Imaging Technol. 2006;22:718-21.

37. Ferring $M$, Claridge $M$, Smith $S A$, et al. Routine preoperative vascular ultrasound improves patency and use of arteriovenous fistulas for hemodialysis: a randomized trial. Clinical journal of the American Society of Nephrology. 2010;5(12):2236-44.

38. Smith GE, Barnes R, Chetter IC. Randomized clinical trial of selective versus routine preoperative duplex ultrasound imaging before arteriovenous fistula surgery. The British journal of surgery. 2014;101(5):469-74.

39. Kosa SD, Al-Jaishi AA, Moist L, et al. Preoperative vascular access evaluation for haemodialysis patients. The Cochrane database of systematic reviews. 2015;9:Cd007013.

40. Georgiadis GS, Charalampidis DG, Argyriou C, et al. The Necessity for Routine Preoperative Ultrasound Mapping Before Arteriovenous Fistula Creation: A Metaanalysis. European journal of vascular and endovascular surgery. 2015;49(5):600-5.

41. Dember LM, Imrey PB, Beck GJ, et al. Objectives and design of the hemodialysis fistula maturation study. American journal of kidney diseases. 2014;63(1):104-12.

42. Lok $C E$, Allon $M$, Moist $L$, et al. Risk equation determining unsuccessful cannulation events and failure to maturation in arteriovenous fistulas (REDUCE FTM I). Journal of the American Society of Nephrology. 2006;17(11):3204-12.

43. Lilly MP, Lynch JR, Wish JB, et al. Prevalence of arteriovenous fistulas in incident hemodialysis patients: correlation with patient factors that may be associated with maturation failure. American journal of kidney diseases. 2012;59(4):541-9.

44. Viecelli AK, Mori TA, Roy-Chaudhury $P$, et al. The pathogenesis of hemodialysis vascular access failure and systemic therapies for its prevention: Optimism unfulfilled. Seminars in dialysis. 2018;31(3):244-57.

45. Humphrey JD. Vascular adaptation and mechanical homeostasis at tissue, cellular, and sub-cellular levels. Cell biochemistry and biophysics. 2008;50(2):53-78.

46. Bode AS, Planken RN, Merkx MA, et al. Feasibility of non-contrast-enhanced magnetic resonance angiography for imaging upper extremity vasculature prior to vascular access creation. European journal of vascular and endovascular surgery. 2012;43(1):88-94. 
47. Bode AS, Leermakers J, Kroon JW, et al. Determination of brachial artery stiffness prior to vascular access creation: reproducibility of pulse wave velocity assessment. Nephrology, dialysis, transplantation. 2012;27(6):2370-6.

48. Huberts W, Heinen SGH, Zonnebeld N, et al. What is needed to make cardiovascular models suitable for clinical decision support? A viewpoint paper. Journal of Computational Science. 2018;24:68-84.

49. van de Vosse FN, Stergiopulos N. Pulse Wave Propagation in the Arterial Tree. Annual Review of Fluid Mechanics. 2011;43(1):467-99.

50. Huberts W, Bode AS, Kroon W, et al. A pulse wave propagation model to support decision-making in vascular access planning in the clinic. Medical engineering \& physics. 2012;34(2):233-48.

51. Bessems D, Giannopapa CG, Rutten MC, et al. Experimental validation of a timedomain-based wave propagation model of blood flow in viscoelastic vessels. Journal of biomechanics. 2008;41(2):284-91.

52. Bessems D, Rutten M, Van De Vosse F. A wave propagation model of blood flow in large vessels using an approximate velocity profile function. Journal of Fluid Mechanics. 2007;580:145-68.

53. Huberts W, Van Canneyt K, Segers $P$, et al. Experimental validation of a pulse wave propagation model for predicting hemodynamics after vascular access surgery. Journal of biomechanics. 2012;45(9):1684-91.

54. Huberts $W$, de Jonge $C$, van der Linden WP, et al. A sensitivity analysis of a personalized pulse wave propagation model for arteriovenous fistula surgery. Part A: Identification of most influential model parameters. Medical engineering \& physics. 2013;35(6):810-26.

55. Huberts $W$, de Jonge $C$, van der Linden WP, et al. A sensitivity analysis of a personalized pulse wave propagation model for arteriovenous fistula surgery. Part B: Identification of possible generic model parameters. Medical engineering \& physics. 2013;35(6):827-37.

56. Bode A, Caroli A, Huberts W, et al. Clinical study protocol for the ARCH project computational modeling for improvement of outcome after vascular access creation. The journal of vascular access. 2011;12(4):369-76.

57. Bode AS, Huberts W, Bosboom EM, et al. Patient-specific computational modeling of upper extremity arteriovenous fistula creation: its feasibility to support clinical decision-making. PloS one. 2012;7(4):e34491. 
General introduction | $\mathbf{2 5}$ 
P

a

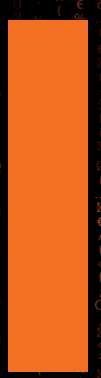

I

t 


\section{CREATING FRAMEWORKS}

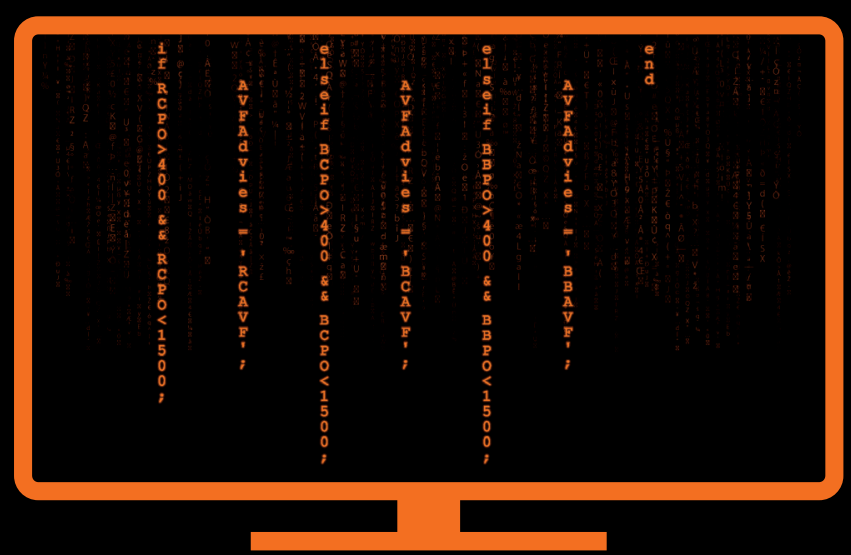



Embargo as requested
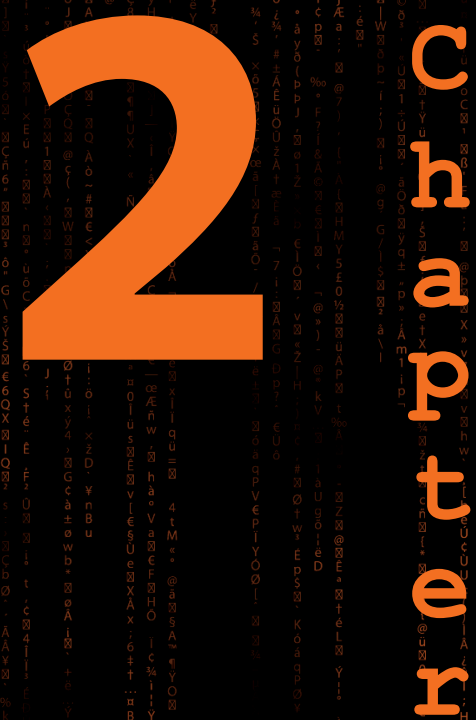

\section{Modeling Strategies in Arteriovenous Fistula Maturation and Functioning: Accomplishments and Improvements}

Niek Zonnebeld ${ }^{1,2}$ Wouter Húberts, ${ }^{1}$ Jan H.M. Tordoir, ${ }_{1}^{2}$ Tammo Delhaas'

In preparation

1. Department of Biomedical Engineering, Maastricht University, Maastricht, The Netherlands 2. Department of Vascular Surgery, Maastricht University Medical Center, Maastricht, The Netherlands 



\section{ABSTRACT}

The arteriovenous fistula (AVF) is the preferred vascular access for hemodialysis patients. Unfortunately, $20-40 \%$ of all constructed AVFs fail to mature (FTM), and are therefore not usable for hemodialysis. AVF maturation importantly depends on postoperative blood volume flow. Predicting patient-specific immediate postoperative flow could therefore support surgical planning. A computational model predicting blood volume flow is available, but the effect of blood flow predictions on the clinical endpoint of maturation (at least $500 \mathrm{~mL} / \mathrm{min}$ blood volume flow, diameter of the venous cannulation segment $\geq 4 \mathrm{~mm}$ ) remains undetermined.

\section{Methods}

A multicenter randomized clinical trial will be conducted in which 372 patients will be randomized (1:1 allocation ratio) between conventional healthcare and computational model-aided decision making. All patients are extensively examined using duplex ultrasonography (DUS) during preoperative assessment (12 venous and 11 arterial diameter measurements; 3 arterial volume flow measurements). The computational model will predict patient-specific immediate postoperative blood volume flows based on this DUS examination. Using these predictions, the preferred AVF configuration is recommended for the individual patient (radiocephalic, brachiocephalic, or brachiobasilic). The primary endpoint is FTM rate at six weeks in both groups, secondary endpoints include AVF functionality and patency rates at 6 and 12 months postoperatively.

\section{Trial registration}

ClinicalTrials.gov (NCT02453412), and ToetsingOnline.nl (NL51610.068.14). 


\section{INTRODUCTION}

The arteriovenous fistula (AVF) is widely recognized as the preferred vascular access for hemodialysis patients. ${ }^{1-4}$ In order to use an AVF for dialysis, it needs to mature after the anastomosis between artery and vein is surgically created. A fistula is considered mature when the flow exceeds $500 \mathrm{~mL} / \mathrm{min}$ and the diameter of the venous cannulation segment is $\geq 4$ mm..$^{5}$ In $20-40 \%$ of all patients, these requirements are not met and the AVFs fail to mature. The maturation process depends heavily on a sufficient flow increase, which fails to occur in case of relatively high vascular resistance to blood flow. The radiocephalic fistula especially, is at risk for FTM due to the smaller diameters and consequently higher resistance in the forearm, as compared to the brachiocephalic or brachiobasilic AVF.6,7

In an effort to aid vascular access surgery to reduce FTM rates, a computer simulation model was developed to patient-specifically predict postoperative blood volume flows. ${ }^{8,9}$ This so-called pulse wave propagation model (PWPM) is based on the physical mass and momentum balance laws, and is able to describe how pressure and flow pulses travel along the arterial tree, effectively relating flow and pressure via model parameters vascular resistance $(\mathrm{R})$, compliance $(\mathrm{C})$, and blood inertia (L). Patient-specific predictions can be made, because the model parameters can be individualized by imputation of patient characteristics, which can readily be acquired using the routine preoperative duplex ultrasonography (DUS) examination used in conventional healthcare. The patient-specific predictions have previously been shown to be accurate enough to support AVF surgery planning. ${ }^{10,11}$ The effect of these predictions on FTM rates, however, remains to be determined.

Here we present our study protocol regarding the multicenter randomized clinical trial, designed to assess the additional value of postoperative blood volume flow predictions in comparison to conventional preoperative assessment only. This trial is registered at ClinicalTrials.gov (NCT02453412) and Toetsingonline.nl (51610.068.14), and is funded by the Dutch Kidney Foundation (NT12.01).

\section{AIMS OF THE STUDY}

The primary aim is to assess whether, in comparison to conventional AVF surgery planning, the FTM rate can be reduced by AVF configuration recommendations based on patient-specific predictions of immediate postoperative blood volume flow. 


\section{Secondary aims}

Secondary aims are to assess (i) the accuracy of blood volume flow predictions when the model is embedded in the conventional healthcare setting; (ii) to what extent and how the recommendations altered clinical decision-making; (iii) AVF functioning (i.e. successful cannulation and effective hemodialysis); (iv) primary, primary assisted, and secondary patency rates; and (v) if the occurrence of high-flow AVFs, and hemodialysis access induced distal ischemia (HAIDI) classification, differs between the study groups.

\section{STUDY DESIGN}

A multicenter randomized clinical trial (two arms with a 1:1 allocation ratio) will be performed in nine Dutch hospitals. In the interventional group the surgeon will receive, in addition to the conventional preoperative work-up, a recommendation on the preferred AVF configuration based on patient-specific receives the conventional preoperative work-up. After surgery, patients are followed for one year to assess maturation, AVF functioning, patency, and development of the AVF conduit (e.g. upper arm cephalic vein diameters, and brachial artery diameters and flow in a BCAVF). In Figure 1, the study visits are presented.

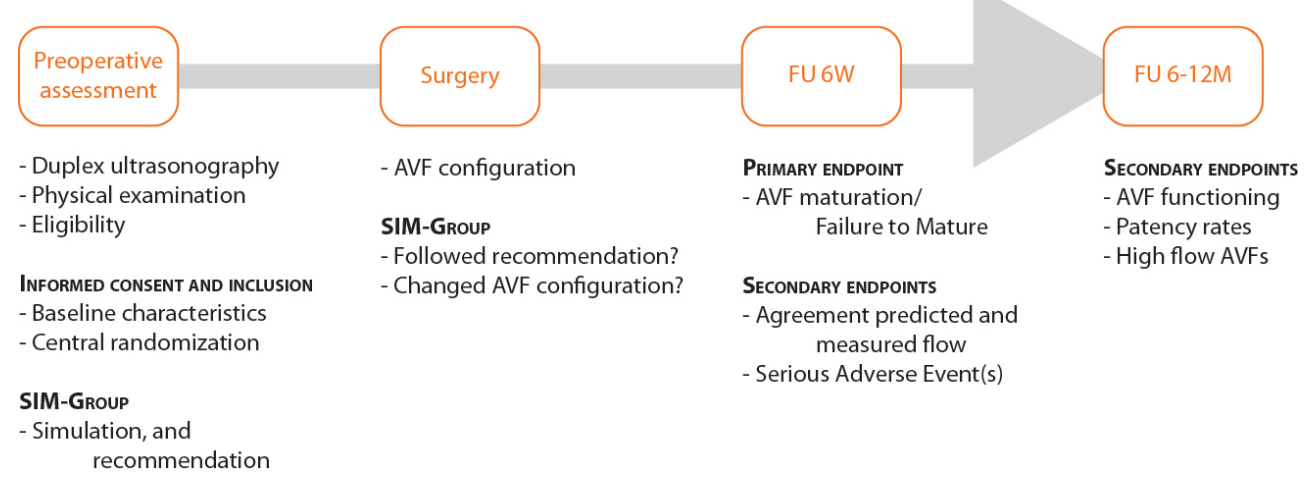

Figure 1 Study flow chart showing the study visits. Specific endpoints for patients that are randomized for the simulation group are captioned by 'SIM-Group'. FU is follow-up visit, at 6 weeks (6W), 6 (6M), or 12 months (12M), with the date of surgery as reference. Serious adverse events (SAEs) are registered until the arteriovenous fistula (AVF) is assessed for maturation. 


\section{Sample size}

A total of 372 patients will be included based on an assumed FTM rate of $25 \%$ in the control group (i.e. $p_{\text {con }}=0.25$ ), and accounting for approximately $10 \%$ patient drop-out. A power calculation for independent proportions (two tailed, $\alpha=0.05, \beta=0.20$ ) is used, for an FTM rate reduction of $50 \%$ in the interventional group (i.e. $p_{\text {sim }}=0.125$ ).

\section{PATIENT INCLUSION AND CRITERIA}

Patients will be recruited at the vascular surgery departments of the participating centers after referral for vascular access creation. Patients will be informed about the study during their preoperative assessment visit, and asked to participate. Patients are given ample time to consider study participation, and the final decision is left at their own discretion. However, if surgical planning requires it, patients can be contacted to inquire about their decision approximately one week after informing them.

\section{Inclusion criteria}

Aged $\geq 18$ years; in need of vascular access for hemodialysis; an autogenous fistula is deemed possible by the vascular surgeon (i.e. either radiocephalic [RCAVF], brachiocephalic [BCAVF] or brachiobasilic [BBAVF]); able to provide written informed consent.

\section{Exclusion criteria}

Patients are excluded from participation in case of prior ipsilateral VA; clinically suspected contra-indications for AVF construction (e.g. high risk of ischemia or cardiac failure); incapable of providing written informed consent.

\section{PREOPERATIVE ASSESSMENT}

The standardized DUS-protocol will be performed by trained and experienced vascular technicians in a warm room $\left(\geq 20^{\circ} \mathrm{C}\right)$, with the patient in supine position. Measurements are performed using the local DUS machines, preferably using linear probes $(10 \mathrm{MHz})$, but for the subclavian measurements a convex transducer can also be used $(7.5 \mathrm{MHz})$ if proper visualization would require so. Brachial systolic and diastolic blood pressures are 
oscillometrically measured at the contralateral arm to allow consistent measurements during follow-up. The ipsilateral digital systolic blood pressure, which is of importance for HAIDI, is measured using a pulse oximeter and small cuff around the base of the third finger. After inflating $30 \mathrm{mmHg}$ above the pressure at which the pulse oximeter reading disappears, the cuff is deflated and the cuff pressure at which the pulse oximeter reading reappears is considered the digital systolic blood pressure.

Venous diameters are measured at discrete locations in the transverse plane, with an antero-posterior and lateral measurement under proximal venous congestion (Figure 2, right panel). The vessels are scanned for continuity and any apparent diameter reduction. The smallest diameter in between measurement locations is also measured. Measurement locations in the forearm are 'distal' ( $\pm 3 \mathrm{~cm}$ proximal of wrist crease), 'proximal' ( $\pm 3 \mathrm{~cm}$ distal from elbow crease), and 'middle' (visual estimation between distal and proximal). In the upper arm, the cephalic vein is measured at 'middle' (halfway from elbow crease to armpit), 'shoulder' (level of humeral head), and 'before deep' ( $\pm 1 \mathrm{~cm}$ distal from the confluence with the deep venous system). The basilic vein is measured at 'distal' ( $\pm 1 \mathrm{~cm}$ proximal from the confluence with the median cubital vein), 'middle', and 'before deep'. The median cubital vein is measured halfway along its course, the subclavian vein is measured by infra- and supraclavicular routes (Figure 3 ).

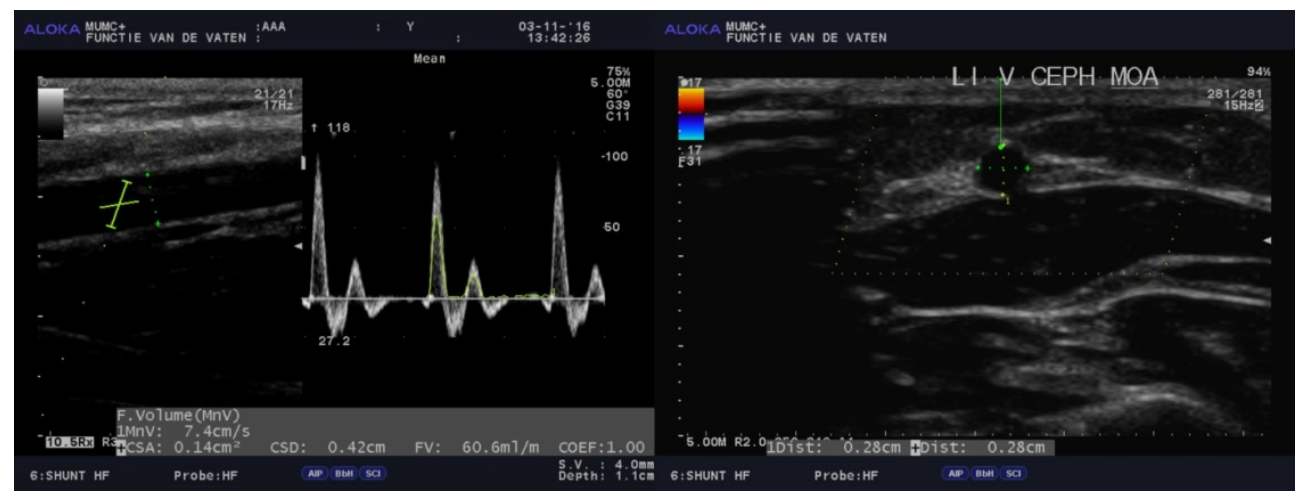

Figure 2 Examples of preoperative duplex ultrasonographic interrogation of the artery (left) and vein (right) in frozen B-mode images. For arterial measures note that the sample volume covers the entire lumen, and for flow velocity measurement the time-averaged mean velocity is automatically traced by the machine. Arterial diameter measurement is in the longitudinal plane by manual inner-to-inner caliper placement. Venous diameter measurements are manually performed in the transverse plane by placing innerto-inner calipers in two directions (i.e. antero-posterior and lateral; see the cross in the right panel).

Arterial diameter measurements are in the longitudinal plane instead of transverse at the same locations as the venous measurements, with the exception of the 'distal' and 
'proximal' measurements around the elbow. These are at least $1 \mathrm{~cm}$ distal (radial and ulnar artery) or proximal (brachial) from the bifurcation (Figure 3). Additionally, blood volume flow is determined at all middle locations (radial, ulnar, and brachial) by multiplying the flow velocity with the cross-sectional area. For flow velocity measurement, the sample volume is set to cover the entire lumen, and the mean velocity is time-averaged (i.e. mean-of-mean; Figure 2, left panel). In case of a high bifurcation, the measurements are performed on the dominant artery (i.e. obvious largest diameter of ulnar or radial, otherwise the radial artery), with an additional diameter measurement and volume flow determination at the middle upper arm of the non-dominant artery. Arteries are also scanned for continuity, and the diameter of any present stenosis is measured.

During follow-up, DUS examinations are performed less extensively with measurements only at those locations incorporated in the vascular access conduit (e.g. brachial artery and upper arm cephalic vein measurements in a BCAVF). The brachial artery blood volume flow is regarded as the AVF flow for all configurations.

\section{Control group: conventional surgery planning}

Patients allocated to the control group are treated in concordance with the Dutch guidelines on vascular access surgery. ${ }^{4}$ In short, the preoperative work-up includes taking a history, physical examination, a preference for the non-dominant arm, and DUS. Physical examination focusses on integrity of the skin (e.g. edema, atrophy, scarring), and quality of (superficial) veins and arteries. The veins are assessed for signs of a sufficiently large, patent, straight segment to allow cannulation, and are percussed to examine the venous wave transmission as a measure for vein quality (i.e. "tap test"). Visible venous collaterals on the arm or chest are indicative of central venous obstructions, and venography is indicated. The arteries are assessed for the quality of the pulses. Using the Allen's test, the collateral circulation is assessed. ${ }^{11}$

The findings at physical examination together with the DUS examination results in the preferred order of AVF configuration of RCAVF, BCAVF, and then BBAVF, given sufficiently large diameters on DUS at the measurement locations. For radiocephalic fistulas, a minimum of $2 \mathrm{~mm}$ for venous and arterial luminal diameters is used, for brachiocephalic or brachiobasilic fistulas the minimal requirement is $3 \mathrm{~mm}$ for artery and vein. ${ }^{4}$ 


\section{Simulation group: computational simulation and surgery planning}

Simulation group-allocated patients are treated in the same fashion as the control group, albeit that the surgeon receives an additional recommendation regarding the ideal AVF configuration based on computer simulations. After imputation of the patientspecific measurements acquired by DUS, the immediate postoperative blood volume flow is predicted for all three configurations. The computational model is described elsewhere in detail. ${ }^{8,9}$ In short, the used model is a pulse wave propagation model (PWPM) based on mass and momentum balance laws applied to the circulatory system. By dividing the vasculature of interest in small individual segments - locally describing the relation between pressure $(p)$ and flow $(q)$ - and then coupling these segments in series, the model captures how pressure and flow pulses travel along the vascular tree. Consequently, the postoperative volume flow can be simulated patient-specifically for multiple AVF configurations (i.e. virtual surgery). Figure 3 gives a schematic representation of such a PWPM for a radiocephalic fistula in the left arm.

Again, the order of preferred configuration is RCAVF, BCAVF, and then BBAVF. However, instead of diameter requirements, a minimum predicted immediate postoperative volume flow of $400 \mathrm{~mL} / \mathrm{min}$ is necessary. This threshold is based on the finding that immediate postoperative volume flow is approximately $60 \%$ of the eventual volume flow for a mature AVF (i.e. $\geq 500 \mathrm{~mL}$ at six weeks), while simultaneously considering possible uncertainties in the flow predictions. ${ }^{12}$

\section{STUDY PROTOCOL ADHERENCE}

Study protocol adherence is maximized by embedding the study in conventional healthcare. However, to ensure strict planning of follow-up visits, the coordinating investigator notifies the participating centers when to call patients. Included patients cannot change allocation; however, they can withdraw their participation at any time. Other reasons for discontinuation are death or access abandonment. In case of early kidney transplantation, functional endpoints (e.g. cannulation, successful dialysis) might not be available, but anatomical and hemodynamical developments are. There are no restrictions to or requirements for concomitant care and/or interventions. However, any relevant intervention to maintain or regain vascular access patency (e.g. percutaneous transluminal angioplasty, thrombectomy) needs to be reported, as well as other relevant medical developments (e.g. percutaneous coronary intervention after AVF creation). 

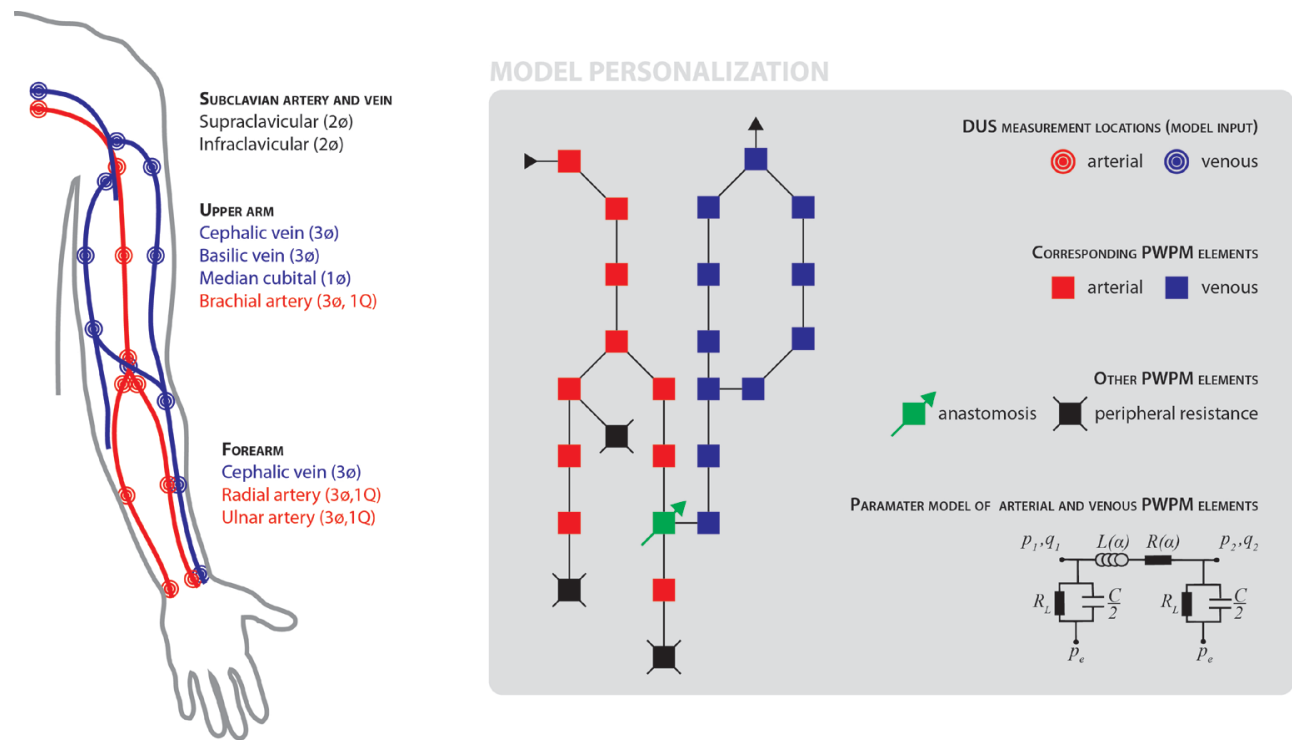

Figure 3 From duplex examination to model personalization. In the left panel, all arterial and venous measurement locations are represented schematically by the red and blue circles respectively. Between parenthesis the number of diameter $(\varnothing)$ and flow $(Q)$ measurements are indicated per segment. See for measurement specifics also Figure 2. The right panel represents the pulse wave propagation model (PWPM) for a radiocephalic arteriovenous fistula configuration. At each red and blue square pressure ( $p$ ) and flow (q) are locally related. These squares consist of two resistors ( $R$; viscous resistance of blood flow, and RL; viscous resistance of blood flow to small side-branches), an inductor ( $\mathrm{L}$; blood inertia), and a capacitator (C; vascular compliance) - see parameter model below right. The green square represents the anastomosis that consists of a nonlinear resistor, and the black squares (peripheral resistance) are three-element windkessel models. DUS: duplex ultrasonography.

\section{STUDY OUTCOMES}

The primary endpoint is the FTM rate at six weeks after AVF construction. A mature AVF is defined as having a minimal volume flow of $500 \mathrm{~mL} / \mathrm{min}$, and a minimal cannulation segment diameter of $4 \mathrm{~mm}$ (e.g. forearm cephalic vein in case of an RCAVF). 


\section{Secondary endpoints}

AVFs are considered functional when six consecutive dialysis sessions can be performed with two needles, and adequate dialysis can be delivered (i.e. minimally $300 \mathrm{~mL} / \mathrm{min}$ dialysis flow, or adequate hemodialysis treatment quantified using urea clearance $(\mathrm{K})$, dialysis time $(\mathrm{t})$, and body water volume $(\mathrm{V})(\mathrm{Kt} / \mathrm{V} \geq 1.4)$ as measured after one of these sessions. The primary, primary assisted, and secondary access patencies are assessed in concordance with the recommended reporting standards. ${ }^{13}$ AVFs are considered 'highflow' $^{\prime}$ in case flow reduction is performed within 12 weeks after construction. Furthermore, HAIDI classification is registered. ${ }^{14}$

\section{DATA ANALYSIS}

All study data are collected via a coded online database (OpenClinica; www.openclinica.nl). Each participating center has their unique study subject list to code participating patients. Electronic patient files serve as source data for study files, and are entered in OpenClinica by the respective professional, or by the coordinating investigator if requested by local study staff. The coordinating investigator assesses the first five patients of each center for completeness and correctness of electronic case report forms (eCRFs), thereafter at random. If necessary, the local principal investigator is notified of any important findings. The trial monitor checks the quality of data at the monitoring visits (see monitoring for specifics) by verifying eCRF data fields with source data.

\section{Statistical methods}

The primary endpoint will be represented in contingency tables and tested for significance using logistic regression analysis. Although proportion comparison would suffice, logistic regression analysis allows consistent reporting when the influence of covariates on maturation is assessed. Covariates are added in a stepwise manner in order of importance according to current literature: age, diabetes, sex, race, AVF-configuration, peripheral arterial disease, and drug use (statins, antithrombotics, ACE-inhibitors). ${ }^{15-19}$

Secondary endpoints will be assessed using logistic regression analysis (high-flow AVFs); Kaplan-Meier survival analysis (patency rates); correlations and Bland-Altman plot (degree of agreement between predicted and measured volume flow); and descriptive statistics with appropriate tests for proportions (recommendation compliance). 
Additional analyses may include the distribution of AVF configuration in the two study groups (e.g. different proportions of RCAVFs), per center recommendation compliance dependent on time, sensitivity and specificity of recommendations for predicting maturation, receiver operating characteristic of the recommendation cut-off value (i.e. $400 \mathrm{~mL} / \mathrm{min}$ volume flow postoperatively).

\section{Data monitoring}

This trial will be independently monitored by the Clinical Trial Center Maastricht. Monitoring activities mainly focus on the preoperative DUS, baseline characteristics, informed consent procedure, and data on the primary endpoint (i.e. six weeks' DUS and clinical data). There is no data safety monitoring board because the study is not blinded, and the definitive decision on which AVF configuration is used is at the discretion of the vascular surgeon.

Interim analyses will be performed after each 100 patients, or yearly, dependent on inclusion rate in the participating centers. Interim analyses mainly focus on the accuracy of volume flow predictions - as they serve as a base for the recommendations - and descriptive statistics regarding the primary endpoint. Additionally, the basic assumption for our sample size calculation will be evaluated at 150 patients. The assumed FTM rate in the control group is based on literature, but might be superseded due to changed hemodialysis demographic factors. There are no specific stopping rules for this study; however, the results and implications of said interim analyses will be thoroughly evaluated by the coordinating study team.

Serious adverse events (SAEs) are registered via OpenClinica, and a notification will then be sent to the coordinating investigator, who, if necessary, contacts the reporting investigator. SAEs are only registered if they occur between VA surgery and assessment of the primary endpoint, and are reported to the approving ethics committee.

\section{ETHICS AND DISSEMINATION}

The present study is approved by the local institutional review board (IRB; Medisch Ethische ToetsingsCommissie azM/UM), and complies with the ICH-GCP guidelines. Participating centers are approved by the same IRB, and local Board of Directors' approval is additionally obtained. Any protocol modification is first approved by said IRB before it is communicated to the local principal investigators at the different sites.

The final results of the randomized controlled trial will be used to draft a manuscript that is offered to a (multidisciplinary) peer-reviewed medical journal for publication. 


\section{DISCUSSION}

Failure to mature remains the major significant drawback of AVF creation. ${ }^{6}{ }^{18}$ AVFs nevertheless continue to be preferred, because of the well-recognized benefits of better patencies, fewer infections, and fewer thrombotic occlusions in comparison to arteriovenous grafts or central venous catheters. A large body of literature is therefore available on determining or mitigating the risk of FTM, including large (cohort) studies trying to identify risk factors, ${ }^{15}, 20,21$ and studies on the advantages of preoperative duplex ultrasonography. $22-24$

AVF maturation strongly depends on hemodynamics, of which AVF blood flow (increase) probably is the decisive factor. Next to the obvious reason of a minimum blood volume flow requirement included in the definitions of a mature AVF, venous enlargement and vascular wall thickening also depend on hemodynamical changes." 25 Venous enlargement and wall thickening are direct hemostatic consequences of the flow increase that follows upon anastomosing the high pressure artery and low pressure vein: the vein enlarges in response to increased wall shear stress (flow), and thickens in response to increased wall stress (pressure). ${ }^{26}$

Given the importance of hemodynamical factors on maturation, one could apply engineering approaches and tools like a PWPM to address the clinical challenge of AVF maturation. This computational model describes how waves propagate through the vascular tree. These waves are caused by the beating heart, and carry information that allows predicting medical interventional outcomes. For extensive reviews on cardiovascular modeling in general we refer to Taylor and Figueroa, ${ }^{27}$ and for pulse wave propagation in particular to Van de Vosse and Stergiopulos. ${ }^{28}$

Patient-specific predictions by means of a PWPM are identified as potentially useful to reduce FTM rates because it complies with the demands of computational models in clinical practice in terms of accuracy, prediction, economy, wellposedness, and utility. ${ }^{29,30}$ The immediate postoperative blood volume flow predictions were previously deemed accurate enough to support decision making. ${ }^{9}{ }^{10}$ Furthermore, the model is economic as it avoids any unnecessary complexity, which was secured by extensive sensitivity analyses. ${ }^{31,32}$ The same sensitivity analyses also secured the well-posedness (i.e. stability) of the model, as minor model input changes are shown not to cause major changes in model outcome. Furthermore, the model could be useful, since the blood volume flow is the important hemodynamic determinant of maturation. Additionally, model input can be obtained by DUS, and recommendation generation can be performed practically in real-time, further endorsing the economy and utility of the model. 
In conclusion, the AVF is recognized as the primary vascular access for hemodialysis patients, but its FTM rate needs to be reduced, especially so for radiocephalic fistulas. Because AVF maturation strongly depends on the physical phenomenon of postoperative blood volume flow, engineering tools could be applied to reduce FTM rates by predicting the immediate postoperative blood volume flow. Though a PWPM was previously shown to be able to accurately predict patient-specific blood volume flow, it is unclear whether implementation of this computational model in conventional healthcare will have positive effects on AVF maturation rates. The aim of the present randomized clinical trial is to supplement vascular access surgery planning with this innovative computer-simulation model, determine how well it is received and assess its effect on AVF maturation rates. 


\section{REFERENCES}

1. National Kidney Foundation Kidney Disease Outcomes Quality Initiative: clinical practice guidelines for vascular access, update 2006. American journal of kidney diseases. 2006;48(Suppl 1):S176-247.

2. Sidawy AN, Spergel LM, Besarab A, et al. The Society for Vascular Surgery: clinical practice guidelines for the surgical placement and maintenance of arteriovenous hemodialysis access. Journal of vascular surgery. 2008;48(5 Suppl):2S-25S.

3. Tordoir J, Canaud B, Haage P, et al. EBPG on Vascular Access. Nephrology, dialysis, transplantation. 2007;22 Suppl 2:ii88-117.

4. NVVH. Richtlijn Shuntchirurgie. 2010.

5. Robbin ML, Chamberlain NE, Lockhart ME, et al. Hemodialysis arteriovenous fistula maturity: US evaluation. Radiology. 2002;225(1):59-64.

6. Huijbregts $\mathrm{HJ}$, Bots $\mathrm{ML}$, Wittens $\mathrm{CH}$, et al. Hemodialysis arteriovenous fistula patency revisited: results of a prospective, multicenter initiative. Clinical journal of the American Society of Nephrology. 2008;3(3):714-9.

7. Allon $M$, Lok CE. Dialysis fistula or graft: the role for randomized clinical trials. Clinical journal of the American Society of Nephrology. 2010;5(12):2348-54.

8. Huberts W, Bode AS, Kroon W, et al. A pulse wave propagation model to support decision-making in vascular access planning in the clinic. Medical engineering \& physics. 2012;34(2):233-48.

9. Bode AS, Huberts W, Bosboom EM, et al. Patient-specific computational modeling of upper extremity arteriovenous fistula creation: its feasibility to support clinical decision-making. PloS one. 2012;7(4):e34491.

10. Caroli A, Manini S, Antiga $L$, et al. Validation of a patient-specific hemodynamic computational model for surgical planning of vascular access in hemodialysis patients. Kidney international. 2013;84(6):1237-45.

11. Wells AC, Fernando B, Butler A, et al. Selective use of ultrasonographic vascular mapping in the assessment of patients before haemodialysis access surgery. The British journal of surgery. 2005;92(11):1439-43.

12. Shemesh D, Goldin I, Berelowitz D, et al. Blood flow volume changes in the maturing arteriovenous access for hemodialysis. Ultrasound in medicine $\&$ biology. 2007;33(5):727-33.

13. Sidawy AN, Gray R, Besarab A, et al. Recommended standards for reports dealing with arteriovenous hemodialysis accesses. Journal of vascular surgery. 2002;35(3):603-10.

14. Tordoir JH, Dammers R, van der Sande FM. Upper extremity ischemia and hemodialysis vascular access. European journal of vascular and endovascular surgery. 2004;27(1):1-5.

15. Lok $C E$, Allon $M$, Moist $L$, et al. Risk equation determining unsuccessful cannulation events and failure to maturation in arteriovenous fistulas (REDUCE FTM I). Journal of the American Society of Nephrology. 2006;17(11):3204-12.

16. Miller CD, Robbin ML, Allon M. Gender differences in outcomes of arteriovenous fistulas in hemodialysis patients. Kidney international. 2003;63(1):346-52. 
17. Lin $\mathrm{SL}$, Huang $\mathrm{CH}$, Chen $\mathrm{HS}$, et al. Effects of age and diabetes on blood flow rate and primary outcome of newly created hemodialysis arteriovenous fistulas. American journal of nephrology. 1998;18(2):96-100.

18. Allon $M$, Robbin ML. Increasing arteriovenous fistulas in hemodialysis patients: problems and solutions. Kidney international. 2002;62(4):1109-24.

19. Ernandez T, Saudan P, Berney $T$, et al. Risk factors for early failure of native arteriovenous fistulas. Nephron Clinical practice. 2005;101(1):c39-44.

20. Lilly MP, Lynch JR, Wish JB, et al. Prevalence of arteriovenous fistulas in incident hemodialysis patients: correlation with patient factors that may be associated with maturation failure. American journal of kidney diseases. 2012;59(4):541-9.

21. Masengu A, Maxwell AP, Hanko JB. Investigating clinical predictors of arteriovenous fistula functional patency in a European cohort. Clinical kidney journal. 2016;9(1):142-7.

22. Wong CS, McNicholas N, Healy D, et al. A systematic review of preoperative duplex ultrasonography and arteriovenous fistula formation. Journal of vascular surgery. 2013;57(4):1129-33.

23. Georgiadis GS, Charalampidis DG, Argyriou C, et al. The Necessity for Routine Preoperative Ultrasound Mapping Before Arteriovenous Fistula Creation: A Metaanalysis. European journal of vascular and endovascular surgery. 2015;49(5):600-5.

24. Kosa SD, Al-Jaishi AA, Moist $L$, et al. Preoperative vascular access evaluation for haemodialysis patients. The Cochrane database of systematic reviews. 2015;9:Cd007013.

25. Allon M, Lockhart ME, Lilly RZ, et al. Effect of preoperative sonographic mapping on vascular access outcomes in hemodialysis patients. Kidney international. 2001;60(5):2013-20.

26. Humphrey JD. Vascular adaptation and mechanical homeostasis at tissue, cellular, and sub-cellular levels. Cell biochemistry and biophysics. 2008;50(2):53-78.

27. Taylor CA, Figueroa CA. Patient-specific modeling of cardiovascular mechanics. Annu Rev Biomed Eng. 2009;11:109-34.

28. van de Vosse FN, Stergiopulos N. Pulse Wave Propagation in the Arterial Tree. Annual Review of Fluid Mechanics. 2011;43(1):467-99.

29. Bode A, Caroli A, Huberts W, et al. Clinical study protocol for the ARCH project computational modeling for improvement of outcome after vascular access creation. The journal of vascular access. 2011;12(4):369-76.

30. Smye SW, Clayton RH. Mathematical modelling for the new millenium: medicine by numbers. Medical engineering \& physics. 2002;24(9):565-74.

31. Huberts $W$, de Jonge $C$, van der Linden WP, et al. A sensitivity analysis of a personalized pulse wave propagation model for arteriovenous fistula surgery. Part B: Identification of possible generic model parameters. Medical engineering \& physics. 2013;35(6):827-37.

32. Huberts $W$, de Jonge $C$, van der Linden WP, et al. A sensitivity analysis of a personalized pulse wave propagation model for arteriovenous fistula surgery. Part A: Identification of most influential model parameters. Medical engineering \& physics. 2013;35(6):810-26. 
P

a

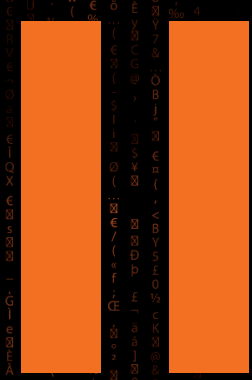

I

t 


\section{EXPLORING ASSUMPTIONS}

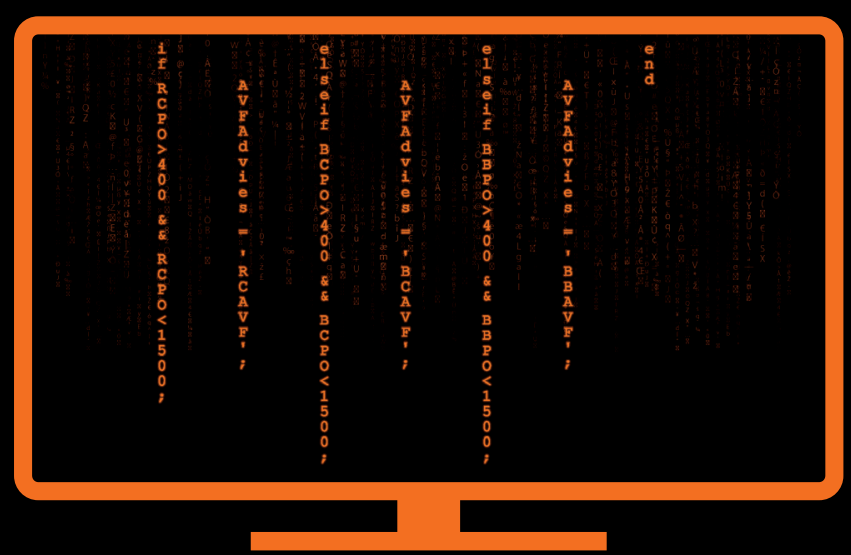




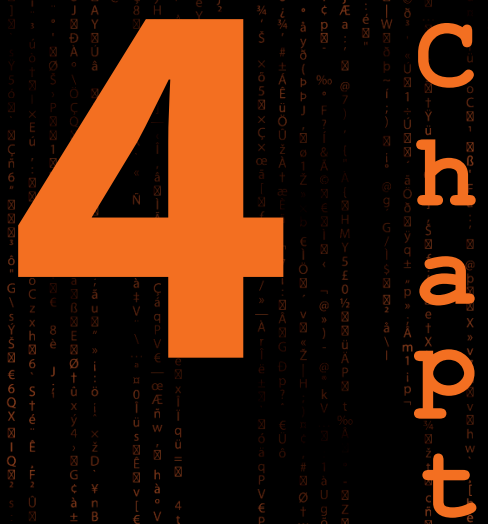

$\Theta$

1

Preoperative Duplex Ultraśnongraphy in Arteriovenous Fistula Creation: Intrat and Interobserver Agreement

Niek Zonnebeld ${ }^{1,2}$ Tommy Maas, ${ }^{1}$ Wouter Huberts, ${ }^{1}$ Magda M. van Loon, ${ }^{2}$ Tammo Delhaas, Jan H.M. Tordoir ${ }^{2}$

Eur JVàsc Endovasc Surg (2017) 54, 613-619

1. Department of Biomedical Engineering, Maastricht University, Maastricht, The Netherlands 2. Department of Vascular Surgery, Maastricht University Medical Center, Maastricht, The Netherlands 


\section{ABSTRACT}

Although clinical guidelines on arteriovenous fistula (AVF) creation advocate minimum luminal arterial and venous diameters, assessed by duplex ultrasonography (DUS), the clinical value of routine DUS examination is under debate. DUS might be an insufficiently repeatable and/or reproducible imaging modality because of its operator dependency. The present study aimed to assess intra- and interobserver agreement of DUS examination in support of AVF surgery planning.

\section{Methods}

Ten end-stage renal disease patients were included to assess intra- and interobserver agreement of preoperative DUS measurements. All measurements were performed by two trained and experienced vascular technicians, blinded to measurement readings. From the routine DUS protocol, representative measurements (venous diameters, and arterial diameters and volume flow in the upper arm and forearm) were selected. For intraobserver agreement the measurements were performed in triplicate, with the probe released from the skin between each. Intraclass correlation coefficients were calculated for intra- and interobserver agreement, and Bland-Altman plots used to graphically display mean measurement differences and limits of agreement.

\section{Results}

Ten patients ( 6 male, $59.4 \pm 19.7$ years) consented to participate, and all predefined measurements were obtained. Intraclass correlation coefficients for intraobserver agreement of diameter measurements were at least $0.90(95 \% \mathrm{Cl} 0.74-0.97$; radial artery). Interobserver agreement was at least 0.83 (0.46-0.96; lateral diameter upper arm cephalic vein). The Bland-Altman plots showed acceptable mean measurement differences and limits of agreement.

\section{Conclusion}

In experienced hands, excellent intra- and interobserver agreement can be reached for the discrete preoperative DUS measurements advocated in clinical guidelines. DUS is therefore a reliable imaging modality to support AVF surgery planning. The content of DUS protocols, however, needs further standardization. 


\section{INTRODUCTION}

The preferred vascular access (VA) for hemodialysis patients is the autologous arteriovenous fistula (AVF). Several international guidelines on vascular access creation recommend using duplex ultrasonography (DUS) to assess the possible AVF configurations preoperatively. ${ }^{1-3}$ It was hypothesized that knowledge of the vascular dimensions would benefit clinical decision making regarding the AVF configuration, as from a hemodynamic point of view, smaller diameters exert higher resistance to blood flow. Indeed, this hypothesized benefit has been confirmed in several clinical studies showing improved AVF maturation rates when vascular dimensions were assessed using DUS and cutoff values were used to decide on a forearm or upper arm AVF.4-6 Consequently, clinical guidelines on AVF creation now advocate minimum arterial and venous luminal diameters for forearm $(\geq 2.0 \mathrm{~mm})$ and upper arm $(\geq 3.0 \mathrm{~mm})$ arteriovenous fistulas. ${ }^{1-3}$

Recently, however, the clinical value of routine DUS in the preoperative work-up for AVF surgery has been doubted in several systematic reviews with meta-analyses. ${ }^{7-9}$ Although a variety of causes for this can be pointed out - especially the considerable variability between studies in DUS examination protocols - the intrinsic properties of DUS examination cannot be ignored. Duplex ultrasonography is operator dependent, and could therefore be an insufficiently repeatable and/or reproducible means of examination. Surprisingly, there are no data available that quantify these intrinsic properties in terms of intra- and interobserver agreement. Previously, it has only been shown that venous diameter measurements are independent of both B-mode image analysis and congestion method. ${ }^{10}, 11$ The former, however, was assessed in healthy subjects with image acquisition (i.e. the major source of error) performed by a single observer. The latter focused on agreement of measurements using pressure cuff versus tourniquet vessel dilatation, and not between observers. Furthermore, both studies were highly standardized and therefore would not have offered agreement measures representative of daily practice. Therefore, the present study was conducted to investigate the intra- and interobserver agreement of routine preoperative DUS examination in support of AVF surgery planning.

\section{METHODS}

This intra- and interobserver agreement study was designed in such a way that it was representative of the routine preoperative work-up at the study institution. From the routine DUS protocol ${ }^{12}$ that complied with clinical guidelines, an arterial and venous 
measurement location was selected in both the forearm and upper arm. In short, the protocol consisted of complete scanning of the veins (cephalic in forearm and upper arm, median cubital, basilic in upper arm, and subclavian) for continuity, and diameters measured at 12 discrete locations. Thereafter, the arteries (subclavian, brachial, radial, and ulnar) were assessed for any stenoses, with diameter measurements performed at 11 discrete locations, including flow measurements at three of them. The selected measurements were performed halfway along the forearm (i.e. halfway between the radial styloid process and elbow crease) or upper arm (i.e. halfway between elbow crease and axilla). These locations were considered representative of the other discrete locations in the forearm or upper arm, and were chosen to prevent difficulties comparing measurements because of anatomical variation between patients (e.g. venous side branches, level of median cubital vein confluence, or arterial bifurcation). The study was embedded in conventional healthcare visits to minimize participation burden for the patients. Measurements for this study were performed after the planned DUS examination, so patients were well acclimatized, by two trained and experienced vascular technicians, who regularly perform preoperative measurements. Institutional review board (IRB) approval was not necessary as the study was used to evaluate routine performance, and participation was at the patient's discretion.

\section{Patients}

All consecutive hemodialysis patients that were planned for a regular DUS examination were informed of the study and asked to participate. Recruitment was completed after 10 patients consented. Both newly referred patients (e.g. pre-dialysis) and patients already on hemodialysis using any type of vascular access were eligible, unless they had a previous VA in both arms.

\section{Measurements}

All measurements were performed as usual (temperature controlled room at $23^{\circ} \mathrm{C}$, with the patient in supine position, vessel dilatation using proximal tourniquet), albeit that each measurement was performed in triplicate to assess intraobserver agreement. Measurement sites were not marked, as this would have positively biased both intraand interobserver agreement. Between each measurement the probe was released from the skin. To ensure independent repeat measures, one of the authors (TM) was present to blind the vascular technician for measurement readings by partially covering the screen in such a way that only caliper or sample volume placement was visible without 
readings. Measurements were performed using the Aloka Prosound a-10 duplex machine (Tokyo, Japan) with a linear probe (10 MHz).

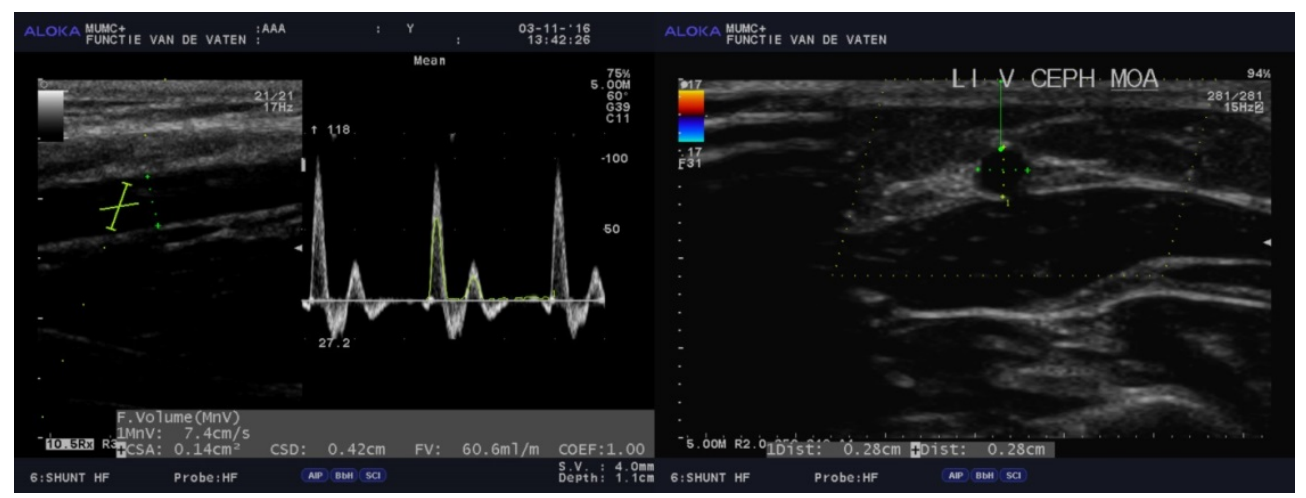

Figure 1 Examples of duplex ultrasonography measurements. On the left the arterial measurement is shown, in which the sample volume covers the entire lumen, and the time-averaged mean velocity is automatically traced by the machine. Diameter measurement is perpendicular to the vessel wall. On the right the cephalic vein is interrogated transversally with diameter measurements antero-posteriorly and mediallaterally.

\section{Venous measurements}

The cephalic vein at the middle of both the forearm and upper arm was measured under compression with a proximal tourniquet. ${ }^{11}$ The vein was visualized transversally and calipers placed in antero-posterior and medial-lateral direction from inner to inner wall (Figure 1, right).

\section{Arterial measurements}

The brachial artery at the middle of the upper arm and the radial artery halfway along the forearm were selected. The arteries were visualized longitudinally to allow diameter and velocity measurements in the same image. For velocity measurement, using pulsed Doppler, the sample volume was set to cover the entire vessel lumen, and the beam angle was kept at $60^{\circ}$. For diameter measurement calipers were placed from inner to inner wall in B-mode (i.e. no visible color Doppler). Volume flow was automatically calculated by the machine using the time-averaged mean velocity (see Figure 1, left). The median of three volume flow determinations was considered as one actual measurement, and the diameter and velocity of this reading was used. For the 
intraobserver study this approach meant that in total nine separate volume flow determinations were performed per location.

\section{Statistical analysis}

Intra- and interobserver agreement were assessed using the intraclass correlation coefficients (ICCs) using ICC.m (version 1.3.1 by Arash Salarian, available via Mathworks File Exchange) in MATLAB version R2016a. For intraobserver agreement the three repeat measurements were used, and for interobserver agreement exclusively the first of three. The latter was to obtain representative measures of agreement, as in daily practice only one measurement is used. Before calculating interobserver ICCs, systematic differences between the two vascular technicians were assessed using the one-sample t-test. Absolute measurement errors were graphically represented using Bland-Altman plots. Simple linear regression was used to assess zero slopes to determine any proportional bias. ICCs were calculated using the " $\mathrm{C}-1$ " model (i.e. consistency in single measures) with a $95 \% \mathrm{Cl}^{13} \mathrm{~A}$ p value $<0.05$ was considered to be statistically significant. ICCs were interpreted as poor $(<0.40)$, fair $(0.40-0.60)$, good $(0.60-0.75)$, or excellent $(0.75-1.00)$ agreement. $^{14}$

Although satisfying point estimates of ICCs were attained, the broad Cls, because of the small sample size, and many separately performed statistical tests urged additional posthoc tests. Firstly, the arterial volume flow, diameter, and velocity, as well as anteroposterior and medial-lateral venous diameter measurements were pooled irrespective of upper arm or forearm location. Consequently, ICCs were obtained on these five samples of 20 measurements. Secondly, the antero-posterior diameter measurements were pooled for forearm and upper arm, irrespective of whether arterial or venous (i.e. 2 samples of 20 measurements). Thirdly, both interobserver biases (i.e. systematic and proportional) were assessed for the second and third measurements to determine whether they were consistently present. Patient characteristics were reported as descriptives.

\section{RESULTS}

Ten patients were included and their characteristics are shown in Table 1. In all patients the complete study protocol could be performed, and all had normal anatomy (i.e. arterial bifurcation at the elbow, with cephalic vein present). Additional scanning time was approximately 30 minutes. For intraobserver agreement 600 measurements were analyzed (10 patients, 10 measurements, in triplicate, by 2 observers), and for 
interobserver agreement 200 measurements (10 patients, 10 measurements, by 2 observers).

Table $1 \quad$ Patient characteristics.

\begin{tabular}{|c|c|}
\hline \multicolumn{2}{|r|}{ N (\%) } \\
\hline Male & $6(60)$ \\
\hline Age (mean $\pm S D)$ & $59.4 \pm 19.7$ \\
\hline On intermittent hemodialysis & $9(90)$ \\
\hline \multicolumn{2}{|l|}{ Comorbidities } \\
\hline Diabetes & $4(40)$ \\
\hline Hypertension & $7(70)$ \\
\hline Hypercholesterolaemia & $5(50)$ \\
\hline Cardiovascular disease & $2(20)$ \\
\hline Cerebrovascular disease & $1(10)$ \\
\hline Peripheral arterial disease & $1(10)$ \\
\hline
\end{tabular}

\section{Intraobserver agreement}

Details of the intraobserver agreement are shown in Table 2. For Observer 1 ICCs ranged from $0.88(0.70-0.97)$ for radial artery velocity (i.e. excellent) to $0.98(0.94-0.99)$ for cephalic vein antero-posterior diameters (i.e. excellent), and for Observer 2 from 0.83 $(0.58-0.95)$ for the radial artery velocity to $0.99(0.96-1.00)$ for the upper arm cephalic vein medial-lateral diameter (both excellent). Axial diameter measurement agreement ranged from 0.91 (0.77-0.97; radial artery) to 0.98 (0.94-0.99; cephalic vein forearm), and from 0.90 (0.74-0.97; radial artery) to 0.97 (0.93-0.99; cephalic vein forearm) for the respective observers (all excellent). 
Table 2 Intraobserver agreement of preoperative duplex ultrasonography measurements.

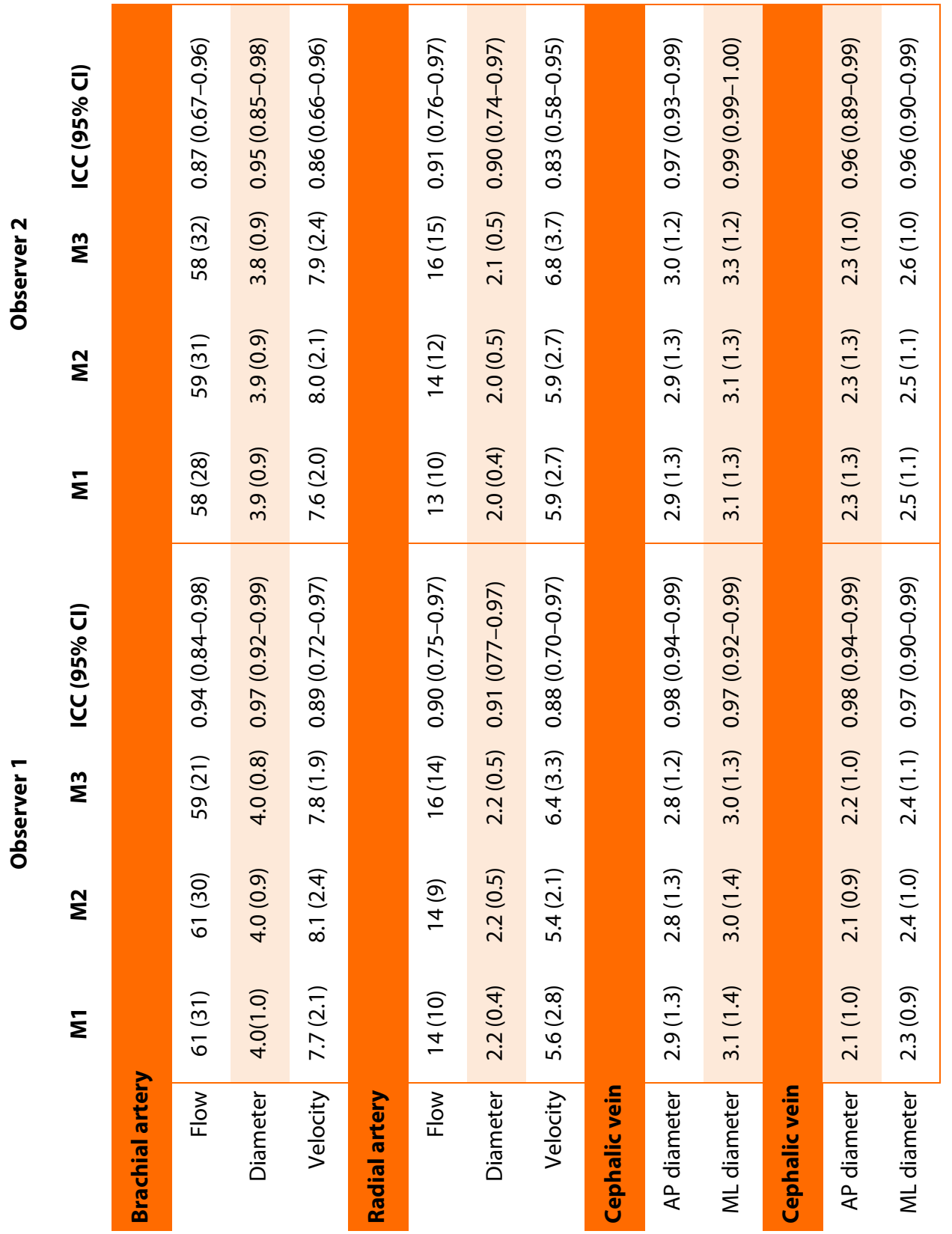

Vessels were assessed at the middle of the upper arm or forearm. Flow was determined using the timeaveraged mean velocity measurement. $\mathrm{M} 1, \mathrm{M} 2$, and $\mathrm{M} 3$ are the respective repeat measurements, reported in mean (SD). Flow is in [mL/min], diameter in [mm], and velocity in [cm/s]. ICC: intraclass correlation coefficient; AP: anteroposterior; ML: medial-lateral. 
Table 3 Interobserver agreement of preoperative duplex ultrasonography measurements.

\begin{tabular}{|c|c|c|c|c|c|}
\hline & $\begin{array}{c}\text { Mean (SD) } \\
\text { Obs1 }\end{array}$ & $\begin{array}{c}\text { Mean (SD) } \\
\text { Obs2 }\end{array}$ & T-test & Zero slope & ICC $(95 \% \mathrm{CI})$ \\
\hline \multicolumn{6}{|l|}{ Brachial artery } \\
\hline Flow & $61(31)$ & $58(28)$ & 0.67 & 0.68 & $0.79(0.35-0.94)$ \\
\hline Diameter & $4.0(1.0)$ & $3.9(0.9)$ & 0.23 & 0.73 & $0.97(0.89-0.99)$ \\
\hline Velocity & $7.7(2.1)$ & $7.6(2.0)$ & 0.82 & 0.80 & $0.74(0.25-0.93)$ \\
\hline \multicolumn{6}{|l|}{ Radial artery } \\
\hline Flow & $14(10)$ & $13(10)$ & 0.71 & 0.88 & $0.91(0.68-0.98)$ \\
\hline Diameter & $2.2(0.4)$ & $2.0(0.4)$ & $0.04^{*}$ & 0.83 & $0.88(0.58-0.97)$ \\
\hline Velocity & $5.6(2.8)$ & $5.9(2.7)$ & 0.67 & 0.94 & $0.69(0.15-0.91)$ \\
\hline \multicolumn{6}{|c|}{ Cephalic vein upper arm } \\
\hline AP diameter & $2.9(1.3)$ & $2.9(1.3)$ & 0.84 & 0.98 & $0.89(0.62-0.97)$ \\
\hline ML diameter & $3.1(1.4)$ & $3.1(1.3)$ & 0.88 & 0.58 & $0.83(0.46-0.96)$ \\
\hline \multicolumn{6}{|c|}{ Cephalic vein forearm } \\
\hline AP diameter & $2.1(1.0)$ & $2.3(1.3)$ & 0.30 & 0.12 & $0.84(0.48-0.96)$ \\
\hline ML diameter & $2.3(0.9)$ & $2.5(1.1)$ & 0.45 & 0.49 & $0.77(0.32-0.94)$ \\
\hline
\end{tabular}

Vessels were assessed at the middle of the upper arm or forearm. Flow was determined using the timeaveraged mean velocity measurement. * Statistically significant in one of three measurements. Flow is in $[\mathrm{mL} / \mathrm{min}]$, diameter in [mm], and velocity in [cm/s]. Obs 1 and -2: Observers 1 and 2 respectively. T-test and Zero slope columns denote the p-value of the respective statistical test. ICC: intraclass correlation coefficient; AP: antero-posterior; ML: medial-lateral.

\section{Interobserver agreement}

Details of the interobserver agreement are shown in Table 3. Acquired ICCs ranged from $0.69(0.15-0.91)$ to $0.97(0.89-0.99)$ for the radial artery velocity and brachial artery diameter measurement, respectively (i.e. fair and excellent). Axial diameter measurement agreement ranged from $0.84(0.48-0.96)$ for forearm cephalic vein to 0.97 (0.90-0.99) for brachial artery (both excellent). There was no evidence of actual systematic or proportional bias in any of the 10 measurements. Statistically significant deviation from zero was only present in one of three repeat measurements when separately assessed. Further post-hoc analyses on pooled upper arm and forearm antero-posterior diameters did not substantially improve ICC point estimates, although $95 \% \mathrm{Cl}$ widths were reduced: $0.93(0.84-0.97)$ respectively $0.82(0.60-0.92)$. Figure 2 shows the Bland-Altman plots for the four antero-posterior diameter measurements, 
all of which show acceptable mean measurement differences and limits of agreement. The limits of agreement were considered acceptable because the largest mean difference (i.e. -0.23 ; solid line of the forearm cephalic vein in Figure 2 ) was $\pm 10 \%$ of the advocated cutoff value for radiocephalic AVF construction. All other measurements showed even better performance.
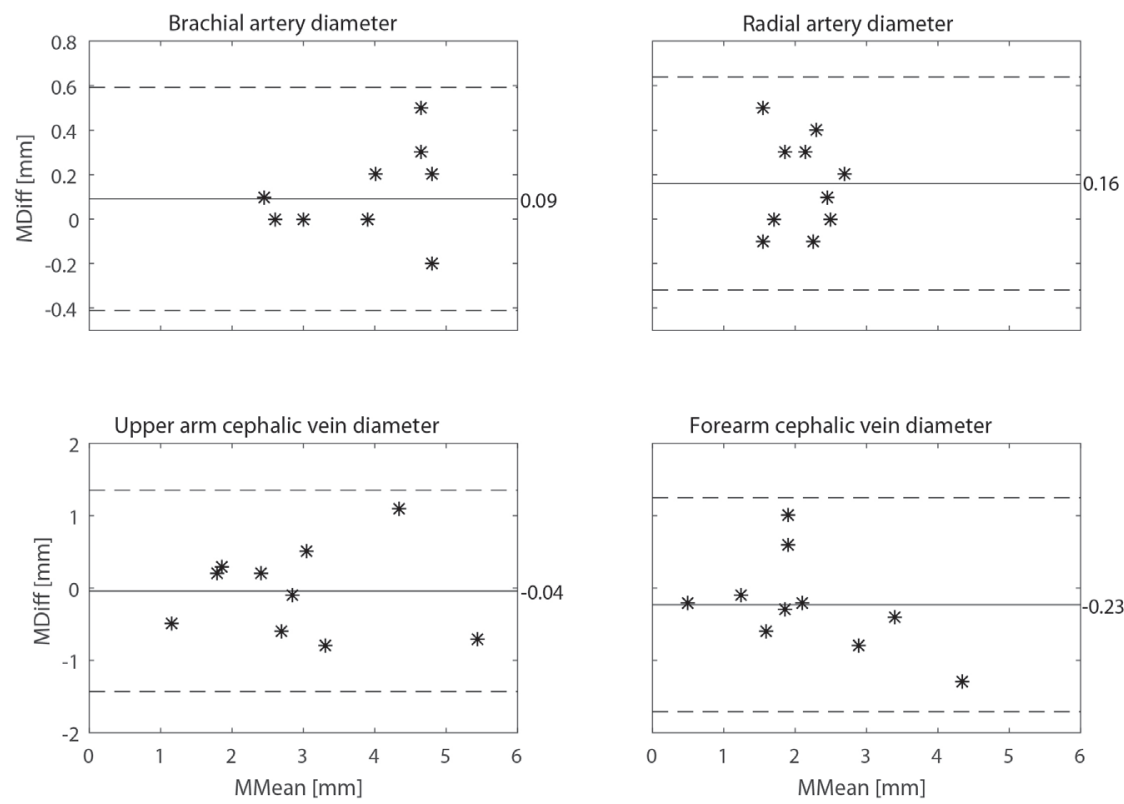

Figure 2 Bland-Altman plots for the four antero-posterior diameter measurements. Solid line is the mean measurement difference between the two observers, dashed lines denote the -2.28 and +2.28 SD limits of agreement. MDiff: measurement difference; MMean: measurement mean.

\section{DISCUSSION}

To the authors' knowledge, this is the first study to assess intra- and interobserver agreement of preoperative vessel assessment using duplex ultrasonography in support of arteriovenous fistula planning. Although the effect of DUS examination aided clinical decision making on clinical outcomes such as fistula maturation has been studied extensively, repeatability and reproducibility remain undetermined. The primary finding of the present study was that DUS examination in experienced hands is a reliable imaging modality to support AVF surgery planning.

By quantifying intra- and interobserver agreement, this study adds to the ongoing debate regarding the (routine) use of DUS in AVF surgery planning. In the late 1990s 
Wong et al., and Malovrh recognized the hemodynamic relevance of vascular dimensions in relation to the clinical outcome of AVF maturation. ${ }^{4-6}$ They identified cutoff values that were associated with improved maturation rates. These observational cohorts and later randomized clinical trials, ${ }^{15-18}$ resulted in guidelines now advocating minimum vein and artery diameters for AVF surgery, assessed by DUS examination. Recently, three systematic reviews with meta-analyses on the subject showed conflicting conclusions, although the most recent one by Georgiadis et al. favored routine DUS. ${ }^{7-9}$ Substantial methodological differences, and the (intrinsic) properties of DUS as an imaging modality might be two complicating factors for such meta-analyses. The RCTs on routine DUS have important differences in their definitions of the control and intervention groups. In the control groups the patients were exclusively physically examined (PE), ${ }^{15,16,19}$ or this examination was selectively supplemented by DUS when PE was inconclusive. ${ }^{17,} 18$ Furthermore, in the DUS groups the extent of the DUS examination, and the cutoff values for AVF construction differed considerably from $1.6 \mathrm{~mm}$ for forearm AVF creation, ${ }^{16} 2 \mathrm{~mm}$ for forearm and upper arm, 1.8 or $2 \mathrm{~mm}$ for forearm and $3 \mathrm{~mm}$ for upper arm. ${ }^{17}$

Although the aforementioned methodological differences are probably the most important explanation for the ongoing debate on routine DUS, DUS itself could also be an inadequate imaging modality to reliably assess vessel diameters in support of AVF surgical planning. However, the present study shows that duplex ultrasonography is a reliable imaging modality. The arterial and venous diameter measurements advocated by the clinical guidelines show excellent intra- and interobserver agreement. Intraobserver agreement was excellent with an ICC of at least 0.90, and even when the lowest $\mathrm{Cl}$ limit applies it was still classified as good (i.e. 0.74). The lowest interobserver agreement was excellent with an ICC of 0.84 at the forearm cephalic vein. Although the point estimates were satisfying, the considerable widths of the Cls led to post-hoc analyses of pooled upper and forearm antero-posterior diameter measurements. The classification varied from fair for the radial artery and forearm cephalic vein to excellent for the brachial artery when the lower $\mathrm{Cl}$ limits applied. After post-hoc analyses, the point estimates per se did not improve ( 0.89 and 0.84 before vs. 0.93 and 0.82 after), but the $\mathrm{Cl}$ widths were reduced to $0.84-0.97$ (at least excellent for upper arm) respectively 0.60-0.92 (at least good for forearm antero-posterior diameter measurements). Forearm diameter agreement was thus at least good, whereas upper arm agreement remained excellent. Although it has been shown that the discrete measurements can be performed reliably, the importance of complete vascular mapping of any arterial stenoses or venous continuity difficulties cannot be underestimated. ${ }^{12}$

The present study has several strengths and limitations. The major strength is that it is both in design data analysis, truly representative of daily practice. Four measurements 
were selected from a standardized preoperative DUS protocol, and were performed as always without any further standardization (e.g. gain settings, exactly measuring distances from anatomical landmarks, time of day of examination). ${ }^{12}$ Intraobserver agreement was optimally assessed by performing the repeat measures at one visit, with the vascular technician blinded to measurement readings. In this way, intraobserver agreement was reliably assessed, as variance in venous diameters notably depends on subject and day rather than observer. ${ }^{11}$ For analysis of interobserver agreement only the first measurement was used. Although using all three would have resulted in better ICCs, these figures would not have been representative of daily clinical practice.

The major limitation of the study is its small sample size on which many separate statistical tests were performed. As no previous data were available that could be used for a sample size determination, a sample size of 10 patients was chosen pragmatically. Although for intraobserver agreement sufficient data were available, interobserver agreement was negatively affected (i.e. wide Cls, type I errors). To overcome this limitation, post-hoc analyses were performed to thoroughly appraise the available data. Antero-posterior diameter measurements were pooled for upper arm or forearm (larger sample, diameters same order of magnitude), and systematic and proportional biases were assessed for all three measurements. As no statistically significant deviation from zero was consistently present in all three measurements, any bias was deemed a type I error. Another limitation is that the present study was set in a tertiary referral center with trained vascular technicians with ample experience in pre-AVF DUS examination, possibly hampering generalizability of the results.

Furthermore, to fully assess the accuracy of DUS measurements, a differently designed or additionally performed study is needed. Accuracy depends not only on the precision of measurements (i.e. random errors) but also the trueness of these measurements (i.e. systematic errors; is $2 \mathrm{~mm}$ really $2 \mathrm{~mm}$ ?). Although the measurements appear to be precise, it remains unclear whether they are true as well. Trueness can be influenced in two ways. First, the duplex machine itself might induce systematic errors. However, because of the spatial resolution of ultrasonography, this bias was assumed to be negligible in relation to the precision. Second, and more importantly, bias can be affected by the fluid status of the patient or preparation before duplex scanning. Clinically, it is very difficult to reliably assess the volume status of patients, and therefore this is not usually done. At present there is no gold standard to clinically assess fluid status. Although bioimpedance measurements are increasingly gaining interest, these are not yet common in daily practice. ${ }^{20}$ Future research on preoperative DUS examination could potentially benefit from bioimpedance guided correction of volume status. With regard to examination preparation, Planken et al. have shown that diameter measurements depend on patient and day, but are congestion type independent 
(pressure cuff vs. tourniquet). ${ }^{11}$ Van Bemmelen et al. on the other hand, have shown that in healthy subjects, out of six different conditions for vein measurement the largest diameter readings can be obtained after warm water submersion with or without pressure cuff $\left(65 \mathrm{mmHg}\right.$ ) inflation. ${ }^{21}$ Korten et al. found that in hemodialysis patients warm water submersion (without tourniquet) resulted in the largest diameters, and concluded that this method should be used. ${ }^{22}$

There are, however, several important side notes. First, indices on intra- and/or interobserver agreement of measurements under different conditions are lacking. Second, only the cephalic and basilic vein at the wrist were examined, and warm water submersion for upper arm veins (i.e. standardization of DUS protocols) might prove very laborious if not impossible. Third, although the maximum diameter gives an indication of distension after AVF construction, the minimum diameter determines vascular resistance and, therefore, is the flow-limiting factor. Using maximum diameters might then overestimate AVF configuration possibilities as it also depends on post-operative flow increase. Fourth, both Van Bemmelen and Korten concluded that the most effective condition should be used, without actually relating it to clinical outcomes. ${ }^{21,} 22$ Given these considerations, the need for standardization of the content of DUS protocols is indisputable. This need is recognized by the aforementioned authors, but the way in which to do this (possibly with minimum and maximum diameters) remains to be determined. ${ }^{21,23}$ For the present study, tourniquet compression was chosen for its adequacy and practicality.

To conclude, the present data show that in experienced hands excellent intra- and interobserver agreements are reached for the discrete preoperative duplex ultrasonographic measurements (i.e. antero-posterior arterial and venous diameters) advocated in the guidelines on vascular access surgery. Duplex ultrasonography is therefore a reliable imaging modality for clinical decision making in arteriovenous fistula creation, and its use is thus justified. Research on the routine use of DUS in AVF surgery planning should make every effort to study clearly defined groups (i.e. PE, PE \pm DUS and PE + DUS), and more importantly use a standardized DUS protocol.

\section{CONFLICT OF INTEREST}

None. 


\section{FUNDING}

None.

\section{ACKNOWLEDGEMENTS}

Sandra Smids-Agricola and Evelien Neven-Plaum, both vascular technicians at Maastricht University Medical Center, for their willingness to have their performance assessed and compared. Sander van Kuijk, clinical epidemiologist, Department of Klinische Epidemiologie en Medical Technology Assessment (KEMTA), Maastricht University, for his support in statistical analysis. 


\section{REFERENCES}

1. NVVH. Richtlijn Shuntchirurgie. 2010.

2. Tordoir J, Canaud B, Haage P, et al. EBPG on Vascular Access. Nephrology, dialysis, transplantation. 2007;22 Suppl 2:ii88-117.

3. Sidawy AN, Spergel LM, Besarab A, et al. The Society for Vascular Surgery: clinical practice guidelines for the surgical placement and maintenance of arteriovenous hemodialysis access. Journal of vascular surgery. 2008;48(5 Suppl):2S-25S.

4. Wong V, Ward R, Taylor J, et al. Factors associated with early failure of arteriovenous fistulae for haemodialysis access. European journal of vascular and endovascular surgery. 1996;12(2):207-13.

5. Malovrh M. Non-invasive evaluation of vessels by duplex sonography prior to construction of arteriovenous fistulas for haemodialysis. Nephrology, dialysis, transplantation. 1998;13(1):125-9.

6. Malovrh M. Native arteriovenous fistula: preoperative evaluation. American journal of kidney diseases. 2002;39(6):1218-25.

7. Wong CS, McNicholas N, Healy D, et al. A systematic review of preoperative duplex ultrasonography and arteriovenous fistula formation. Journal of vascular surgery. 2013;57(4):1129-33.

8. Georgiadis GS, Charalampidis DG, Argyriou C, et al. The Necessity for Routine Preoperative Ultrasound Mapping Before Arteriovenous Fistula Creation: A Metaanalysis. European journal of vascular and endovascular surgery. 2015;49(5):600-5.

9. Kosa SD, Al-Jaishi AA, Moist L, et al. Preoperative vascular access evaluation for haemodialysis patients. The Cochrane database of systematic reviews. 2015;9:Cd007013.

10. Planken RN, Keuter $\mathrm{XH}$, Kessels AG, et al. Forearm cephalic vein cross-sectional area changes at incremental congestion pressures: towards a standardized and reproducible vein mapping protocol. Journal of vascular surgery. 2006;44(2):353-8.

11. Planken RN, Keuter $\mathrm{XH}$, Hoeks $A P$, et al. Diameter measurements of the forearm cephalic vein prior to vascular access creation in end-stage renal disease patients: graduated pressure cuff versus tourniquet vessel dilatation. Nephrology, dialysis, transplantation. 2006;21(3):802-6.

12. Zonnebeld N, Huberts W, van Loon MM, et al. Preoperative computer simulation for planning of vascular access surgery in hemodialysis patients. The journal of vascular access. 2017;18(Suppl. 1):118-24.

13. McGraw KO, Wong SP. Forming inferences about some intraclass correlation coefficients. Psychological methods. 1996;1(1):30.

14. Cicchetti DV. Guidelines, criteria, and rules of thumb for evaluating normed and standardized assessment instruments in psychology. Psychological assessment. 1994;6(4):284.

15. Mihmanli I, Besirli K, Kurugoglu S, et al. Cephalic vein and hemodialysis fistula: surgeon's observation versus color Doppler ultrasonographic findings. Journal of ultrasound in medicine. 2001;20(3):217-22. 
16. Nursal TZ, Oguzkurt L, Tercan F, et al. Is routine preoperative ultrasonographic mapping for arteriovenous fistula creation necessary in patients with favorable physical examination findings? Results of a randomized controlled trial. World journal of surgery. 2006;30(6):1100-7.

17. Ferring $M$, Claridge $M$, Smith $S A$, et al. Routine preoperative vascular ultrasound improves patency and use of arteriovenous fistulas for hemodialysis: a randomized trial. Clinical journal of the American Society of Nephrology. 2010;5(12):2236-44.

18. Smith GE, Barnes R, Chetter IC. Randomized clinical trial of selective versus routine preoperative duplex ultrasound imaging before arteriovenous fistula surgery. The British journal of surgery. 2014;101(5):469-74.

19. Zhang $Z$, Wang $X$, Zhang $Z$, et al. Hemodynamic evaluation of native arteriovenous fistulas for chronic hemodialysis with color Doppler ultrasound. Chin J Med Imaging Technol. 2006;22:718-21.

20. Zhu F, Rosales $L$, Kotanko P. Techniques for assessing fluids status in patients with kidney disease. Current opinion in nephrology and hypertension. 2016;25(6):473-9.

21. van Bemmelen PS, Kelly P, Blebea J. Improvement in the visualization of superficial arm veins being evaluated for access and bypass. Journal of vascular surgery. 2005;42(5):957-62.

22. Korten E, Spronk S, Hoedt MT, et al. Distensibility of forearm veins in haemodialysis patients on duplex ultrasound testing using three provocation methods. European journal of vascular and endovascular surgery. 2009;38(3):375-80.

23. Planken NR, Leiner T, Tordoir JH. Regarding "Improvement in the visualization of superficial arm veins being evaluated for access and bypass". Journal of vascular surgery. 2006;43(3):645-6; author reply 6. 
Preoperative DUS Examination: Intra- And Interobserver Agreement | 97 



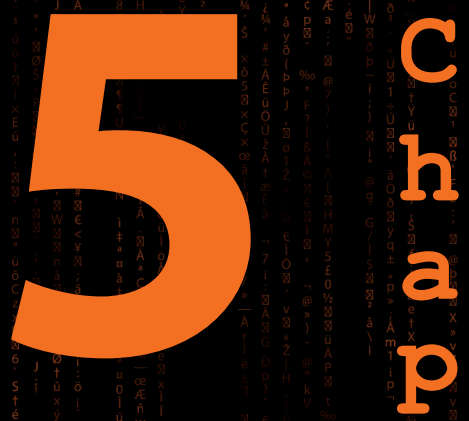

Natural Vascular Remodeling After Arteriovenous Fistula Creation in Dialysis Patients With and Without Previous Ipsilateral Vascular Access

Niek Zonnebeld ${ }^{1,2}$ Wouter Huberts, ${ }^{1}$ Magda M. van Loon, ${ }^{2}$ Tammo Delhaas, Jan H.M. Tordoir ${ }^{2}$

Under review

1. Department of Biomedical Engineering, Maastricht University, Maastricht, The Netherlands 2. Department of Vascular Surgery, Maastricht University Medical Center, Maastricht, The Netherlands 


\section{ABSTRACT}

The aim of the study was to observe the natural hemodynamical changes after arteriovenous fistula (AVF) creation in hemodialysis patients with and without a previous ipsilateral vascular access.

\section{Methods}

Retrospective, single center cohort study. Patient demographics were registered and pre- and postoperative vessel ultrasonography examinations were performed at regular follow up intervals. Arteriovenous fistula outcome in terms of vessel diameter and access flow enhancement were determined in radiocephalic, brachiocephalic and brachiobasilic AVFs.

\section{Results}

331 patients (median age 66 yr, 60\% male) with 366 new autologous AVFs were studied, of which 112 patients had a previous ipsilateral VA. Patients with a previous ipsilateral VA had significantly greater preoperative brachial artery diameter $(4.4 \mathrm{~mm})$ and flow $(105$ $\mathrm{mL} / \mathrm{min})$, and basilic vein diameter $(4.9 \mathrm{~mm})$, in comparison to patients without a previous ipsilateral VA $(4.0 \mathrm{~mm}, 54 \mathrm{~mL} / \mathrm{min}$, and $4.3 \mathrm{~mm}$ respectively). For all AVF configurations these differences gradually disappeared after AVF creation over the course of three months. The hemodynamical changes reached a plateau at three months, and were significantly accelerated in patients with a previous ipsilateral VA. There were no differences in occurrence of primary failure or high flow complications between both groups.

\section{Conclusion}

Arteriovenous fistulae show hemodynamical and remodeling changes up to three months postoperatively. Previous ipsilateral VAs may initiate vessel preconditioning, and accelerate the observed hemodynamical changes after AVF creation. This preconditioning, however, does not result in a beneficial or detrimental effect on VA functioning. 


\section{INTRODUCTION}

Autologous arteriovenous fistulae (AVFs) for hemodialysis undergo profound anatomic and hemodynamical changes after surgical creation (i.e. dilatation and vessel remodeling). On the one hand these adaptation changes are necessary to ensure a matured AVF for safe needle cannulation and sufficient blood flow needed for dialysis treatment, while on the other hand they pose a risk for complications related to the altered hemodynamics. AVFs with anastomosis of small vessels (i.e. the radiocephalic AVF) usually have a low risk of hemodynamic complications, but a high risk for failure to mature (FTM) or primary failure. ${ }^{1-3}$ Conversely, upper arm brachiocephalic (BC) and brachiobasilic (BB) AVFs typically generate higher flows and therewith pose an increased risk of high flow complications like heart failure and distal ischemia. ${ }^{4,5}$

Typically the first six weeks after AVF creation are considered the maturation time in which the necessary maturation processes occur. ${ }^{6,7}$ Yet recently it has been shown that the median functional maturation time may take up to 13 weeks, ${ }^{8}$ and fistula vein size and flow may increase beyond that time as well. ${ }^{9}$ The hemodynamical changes after AVF surgery, however, are usually studied only during these first six weeks, and these studies generally include radiocephalic fistulae only. ${ }^{10-14}$ Furthermore, no data are available on how these changes are affected by any previous ipsilateral vascular access (VA). A vascular access may initiate vessel pre-conditioning, which in turn can influence the postoperative hemodynamics after creation of a new ipsilateral fistula. Consequently, patients with previous ipsilateral VAs might have a lower risk on primary failure, and/or an increased risk for high flow complications.

The present study aims to fill the gaps described above by determining the long-term (i.e. beyond six weeks postoperatively) hemodynamical changes after AVF creation. In addition, it aims to assess if and how these changes are affected by a previous ipsilateral $V A$, and the consequent influence thereof on primary failure and high flow complications.

\section{METHODS}

A retrospective analysis of all vascular access procedures for hemodialysis between January $1^{\text {st }} 2007$ and January $1^{\text {st }} 2017$ was performed in a single tertiary referral center. All surgical procedures were extracted from the electronic health records (EHR) for patients by means of a vascular access billing code. This list was then manually crossreferenced for completeness and correctness. Exclusively patients that received an upper extremity autologous AVF for hemodialysis were eligible for analysis. 
Arteriovenous fistulae were followed from the date of surgery until any event or intervention influencing the natural hemodynamics of the fistula or ascertainment thereof. These events included primary failure (i.e. thrombosis or creation of a new AVF configuration), any surgical or endovascular intervention that alters the initial AVF conduit, transfer to a different hospital, death, or loss to follow-up. A stenotic event was scored when a percutaneous transluminal angioplasty (PTA) was performed. Ischemic or high flow events were scored when respectively a procedure to increase distal perfusion or reduce AVF flow was performed (e.g. Revascularization Using Distal Inflow [RUDI], Distal Revascularization and Interval Ligation [DRIL], banding, etc.). Institutional review board approval was waived due to the retrospective nature of the study on routinely acquired data.

\section{Data extraction and definitions}

The following baseline characteristics were extracted from the EHR: date of birth, date of death (if applicable), sex, cause of end-stage renal disease (ESRD), dialysis dependency, comorbidity (i.e. diabetes, peripheral arterial disease, hypertension, hypercholesterolemia, cardiovascular, cerebrovascular), antiplatelet or -coagulation therapy, any previous ipsilateral VA, and the results of the preoperative vascular mapping by duplex ultrasonography (DUS). The respective comorbidity was deemed present or not present when documented in the patient's history, surgical records, (vascular) laboratory findings, and/or in case any relevant drug was prescribed. Diabetes was subdivided in insulin dependent and independent.

Data extracted from surgical procedures were: date of surgery, AVF configuration, side, and anesthesia type (i.e. local, regional/plexus block, general). Postoperative DUS measurements and the end of follow-up date and reason were extracted respectively determined. Following common practice, the mid upper arm brachial artery volume flow was considered the AVF flow for all configurations. ${ }^{15}$ The mid forearm (cephalic vein) and mid upper arm (cephalic or basilic vein) vein diameters were used to assess AVF vein diameter. AVF vein and flow are used together to assess AVF maturation according to two sets of DUS-based AVF maturation criteria at the various time points. The University of Alabama at Birmingham (UAB) criteria are an AVF vein diameter $\geq 4 \mathrm{~mm}$, and an AVF flow $\geq 500 \mathrm{~mL} / \mathrm{min}^{9}{ }^{9}$ The Rule of $6 \mathrm{~s}$ (Ro6s) uses values of $\geq 6 \mathrm{~mm}$, and $\geq 600 \mathrm{~mL} / \mathrm{min}$ respectively. ${ }^{6}$ 


\section{Procedures}

Preoperatively, patients received an extensive DUS examination. Together with the findings of physical examination that includes determination of the quality of superficial veins (e.g. straight or tortuous), skin quality, and arterial pulsations (including Allen test) the operative procedure was planned. The most distal AVF configuration was preferred when minimal vessel diameters were met (i.e. 2.0 or $3.0 \mathrm{~mm}$ for artery and vein in the forearm and upper arm respectively), and in absence of contra-indications such as short or tortuous cannulation segments, or extensive skin defects.

\section{Duplex ultrasonography}

A standardized DUS protocol was performed both pre- and postoperatively by trained and experienced vascular technicians. The patient was in a warm room $\left( \pm 23^{\circ} \mathrm{C}\right)$ in supine position. During the study period the Aloka Prosound a10 (Tokyo, Japan) DUS machine with a $10 \mathrm{MHz}$ linear transducer was used. The preoperative DUS protocol entails 12 discrete venous diameter measurements under proximal congestion, 11 discrete arterial diameter measurements, and 3 discrete arterial blood volume flow measurements. In between the discrete measurements the veins are scanned for their continuity, and arteries are scanned for stenoses..$^{15}$ For the present study, the measurement locations were in the forearm halfway between the wrist and elbow crease, and for the upper arm halfway the elbow crease and armpit. Venous diameters were assessed transversally. Arterial diameters were assessed longitudinally to allow concurrent Doppler velocity measurements. Flow measurements were performed with the sample volume set to cover the entire vessel lumen, and the time-averaged mean velocity was used for automatic flow volume calculation. The postoperative measurements were limited to the AVF conduit vessels.

\section{Operative procedures}

Operative procedures for the various AVF configurations were standardized, using endto-side anastomoses with running polypropylene (prolene) 6 or 7-0 sutures. When a high arterial bifurcation was present, usually the largest artery was used for AV anastomosis in BC- or BBAVFs. For RCAVFs, a longitudinal or transverse skin incision was made $5 \mathrm{~cm}$ proximally from the wrist, and the cephalic vein and radial artery were dissected from their surrounding tissues. After distal cephalic vein transection it was mobilized and anastomosed (length 8 to $10 \mathrm{~mm}$ ) with the radial artery. BCAVFs required a transverse incision $2 \mathrm{~cm}$ distally from the elbow crease. The subcutaneous cubital and 
cephalic veins were dissected, and the lacertus fibrosis transversely opened. Then, the brachial artery was dissected, and consequently a running suture anastomosis (length $7 \mathrm{~mm}$ ) with the cephalic vein made. Occasionally a brachiocubital AVF was constructed. In this case the perforating instead of the cephalic vein was used for anastomosis with the brachial artery. All BBAVFs were performed in a one-stage procedure. Through an interrupted medial skin incision the basilic vein in the upper arm was dissected and transected as distally as possible. The brachial artery was dissected after a transverse skin incision $2-5 \mathrm{~cm}$ proximally from the elbow crease. The basilic vein was pulled through an anterolateral subcutaneous tunnel, and anastomosis $(7-10 \mathrm{~mm})$ performed with the brachial artery.

\section{Follow up}

Follow-up visits with clinical VA assessment and DUS were scheduled after 2, 6, 12 and 24 weeks, and at six month intervals thereafter. Occasionally a direct postoperative flow measurement was available (i.e. $0-2$ days) as part of a clinical trial.

\section{Statistical analysis}

Continuous variables are represented as mean (SD) or median (IQR) where appropriate, and tested for significance with the (independent or dependent) t-test or the MannWhitney $U$ test respectively. Categorical variables are represented as count (\%), and tested for significance using the Chi-square test. Additionally, AVF vein diameter and brachial artery flow development is visualized using boxplots. A two-sided p-value $<0.05$ is considered statistically significant. All statistics were performed using SPSS version 22, boxplots were created with MATLAB R2016a.

\section{RESULTS}

During the study period 366 autologous AVFs were created in 331 unique patients (median age 66 years, $60 \%$ male). Patient demographics are presented in Table 1. Out of 366 AVFs, 112 AVFs were created in arms with a previous VA, whereas 254 were created in arms without any previous ipsilateral VA. The BBAVF was the predominant configuration ( $n=69 ; 62 \%$ ) in group of patients with a previous VA, while the three conventional AVF configurations were equally distributed in the group without a previous ipsilateral VA. Interestingly enough, some RCAVFs were constructed ( $n=6 ; 5 \%)$ in the group that previously had an AVF. There were four brachiocubital AVFs created in 
four patients with no previous ipsilateral VA. Because of this small number of patients without comparison, these patients were excluded from further analysis.

Table 1 Patient demographics $(\mathbf{n}=\mathbf{3 3 1})$.

\section{Demographic}

\begin{tabular}{r|cc|} 
& With $(\mathbf{n = 8 6})$ & Without $(\mathbf{n = 2 4 5})$ \\
\hline Age (years) $^{*}$ & $61(47-68)$ & $69(55-77)$ \\
Male* $^{*}$ & $43(50)$ & $156(64)$
\end{tabular}

\section{Cause of ESRD*,}

Unknown

Glomerulonephritis

Interstitial nephritis

Cystic kidney diseases

Other congenital and hereditary kidney diseases

Renal vascular disease, excluding vasculitis

Diabetes Mellitus

Other multisystem diseases

Others

$16(19)$

$16(19)$

$13(15)$

$12(14)$

$16(19)$

$6(7)$

$6(7)$

$9(11)$

7 (8)

$1(1)$
$20(8)$

$28(11)$

$14(6)$

25 (10)

$2(1)$

$78(32)$

$43(18)$

$24(10)$

11 (5)

\begin{tabular}{|c|c|c|}
\hline \multicolumn{3}{|l|}{ Diabetes } \\
\hline Not insulin dependent & $5(6)$ & $20(8)$ \\
\hline Insulin dependent & $16(19)$ & $56(23)$ \\
\hline \multicolumn{3}{|l|}{ Other comorbidity } \\
\hline Hypertension* & $70(81)$ & $237(96)$ \\
\hline Peripheral arterial disease ${ }^{*}$ & $7(8)$ & $52(21)$ \\
\hline Cardiovascular disease & $35(41)$ & $123(50)$ \\
\hline Cerebrovascular disease & $11(15)$ & $39(16)$ \\
\hline Hypercholesterolemia* & $35(41)$ & $159(65)$ \\
\hline \multicolumn{3}{|l|}{ Dialysis ${ }^{*}, 2$} \\
\hline Predialysis & $16(20)$ & $96(40)$ \\
\hline Hemodialysis via CVC & $57(72)$ & $135(56)$ \\
\hline Hemodialysis via other VA & $3(4)$ & $2(1)$ \\
\hline Peritoneal dialysis & $3(1)$ & $10(4)$ \\
\hline Unknown & $7(8)$ & $2(1)$ \\
\hline \multicolumn{3}{|l|}{ Anticoagulant use } \\
\hline Antiplatelet & $22(29)$ & $96(42)$ \\
\hline Vitamin K antagonist & $14(19)$ & $36(12)$ \\
\hline
\end{tabular}

All values but age are reported as number (percentage). Age is reported as median (IQR). Unknown cause of end-stage renal disease (ESRD) includes both unknown cause (inconclusive diagnostics) as well as not documented (especially for referred patients). ${ }^{*}$ denotes a statistically significant difference between both groups. ${ }^{1}$ Primarily due to higher proportions of cystic, and other congenital and hereditary kidney diseases, and lower proportion of renal vascular disease in the with group. ${ }^{2}$ In the with group fewer patients are predialysis, and more on hemodialysis via a central venous catheter (CVC), albeit that 3 cells had an expected count less than 5 . VA: vascular access. 
Overall median follow-up time did not differ significantly between the two groups: 132 (53-285) versus 133 (56-386) days for patients with and without any previous VA respectively $(p=0.341)$. Reasons for ending of follow-up were different between the two groups ( $p<0.001)$, and the standardized residuals indicate that this was primarily due to a difference in the number of patient transfers (Table 2). Primary failure (10 and $4 \%$ respectively) and high flow (8 and 11\%) rates were similar in both groups (all standardized residuals $<1.96$, data not shown). Overall median follow-up time was 154 (67-545), 141 (55-376), and 119 (52-263) days for RC-, BC- and BBAVF respectively $(p=0.094)$.

Table 2 Reasons for and timing of end of follow-up.

With $(n=112)$

Without ( $\mathbf{n = 2 5 4 )}$

\begin{tabular}{|r|cc|cc|}
\hline & Counts (\%) & Days (IQR) & Counts (\%) & Days (IQR) \\
Primary failure & $4(4)$ & 14 & $26(10)$ & $81(34-125)$ \\
Stenosis & $44(39)$ & $162(92-300)$ & $105(42)$ & $133(70-267)$ \\
Ischemia/High flow & $12(11)$ & $142(79-271)$ & $20(8)$ & $151(64-412)$ \\
Patient transfer & $30(27)$ & $55(15-153)$ & $25(10)$ & $48(0-86)$ \\
Death & $6(5)$ & $131(61-831)$ & $29(12)$ & $309(103-1463)$ \\
Loss to follow-up & $5(5)$ & $135(32-468)$ & $19(8)$ & $339(38-1156)$ \\
Other & $7(6)$ & $144(70-1128)$ & $11(4)$ & $101(13-606)$ \\
\cline { 2 - 4 }
\end{tabular}

Reasons are statistically significant different between the two groups. Chi-square $=25.740, p<0.001$, minimum expected count is 5.54. Considering standardized residuals, this effect is primarily due to Patient transfer. Days to for the various reasons did not differ between groups assessed with the Mann-Whitney $U$ test $(p=0.341)$.

Preoperative DUS showed significant differences between both groups in terms of greater brachial artery diameter and flows (4.4 versus $4.0 \mathrm{~mm}$ and 105 versus $54 \mathrm{~mL} / \mathrm{min}$ respectively), and upper arm basilic vein diameter ( 4.9 versus $4.3 \mathrm{~mm}$ ) in patients with a previous VA (Table 3a). Furthermore, patients with a previous VA had higher ulnar artery flows ( 23 versus $10 \mathrm{~mL} / \mathrm{min}$ ). For patients without a previous VA, female patients had smaller arterial diameters compared to men, which may explain the lower number of RCAVFs created in women compared to men (Table 3b). 
Table 3a Preoperative measurements for patients with and without a previous ipsilateral VA, and eventual AVF configuration.

\begin{tabular}{|c|c|c|c|}
\hline & With $(n=112)$ & Without $(n=254)$ & p-value \\
\hline \multicolumn{4}{|l|}{ Upper arm } \\
\hline Brachial artery $(\mathrm{d})$ & $4.4(1.3)$ & $4.0(0.8)$ & $0.024^{*}$ \\
\hline Brachial artery $(q)$ & $105(144)$ & $54(31)$ & $0.002^{*}$ \\
\hline Cephalic vein & $3.3(1.5)$ & $3.2(1.2)$ & 0.752 \\
\hline Basilic vein & $4.9(1.5)$ & $4.3(1.3)$ & $0.003^{*}$ \\
\hline \multicolumn{4}{|l|}{ Forearm } \\
\hline Radial artery $(\mathrm{d})$ & $2.3(1.1)$ & $2.1(0.5)$ & 0.360 \\
\hline Radial artery (q) & $22(31)$ & $12(9)$ & 0.109 \\
\hline Ulnar artery $(\mathrm{d})$ & $2.1(0.5)$ & $1.9(0.6)$ & 0.138 \\
\hline Ulnar artery $(q)$ & $23(12)$ & $10(8)$ & $<0.001^{*}$ \\
\hline Cephalic vein & $2.8(1.2)$ & $2.4(0.9)$ & 0.052 \\
\hline \multicolumn{4}{|l|}{ AVF Configuration" } \\
\hline RCAVF & $6(5)$ & $85(34)$ & \\
\hline BCAVF & $37(33)$ & $80(32)$ & \\
\hline BBAVF & $69(62)$ & $85(34)$ & \\
\hline Brachiocubital\$ & 0 & $4(2)$ & \\
\hline
\end{tabular}

Table 3b Preoperative measurements and eventual AVF configurations in virgin arms per sex.

\begin{tabular}{|c|c|c|c|}
\hline & Male $(n=159)$ & Female $(n=95)$ & p-value \\
\hline \multicolumn{4}{|l|}{ Upper arm } \\
\hline Brachial artery $(\mathrm{d})$ & $4.3(0.7)$ & $3.6(0.7)$ & $<0.001^{*}$ \\
\hline Brachial artery (q) & $61(34)$ & $44(20)$ & $<0.001^{*}$ \\
\hline \multirow{2}{*}{$\begin{array}{r}\text { Cephalic vein } \\
\text { Basilic vein }\end{array}$} & $3.3(1.3)$ & $3.1(1.0)$ & 0.122 \\
\hline & $4.5(1.4)$ & $4.2(1.2)$ & 0.105 \\
\hline \multicolumn{4}{|l|}{ Forearm } \\
\hline Radial artery (d) & $2.2(0.5)$ & $2.0(0.6)$ & $<0.001^{*}$ \\
\hline Radial artery (q) & $13(9)$ & $10(8)$ & $0.006^{*}$ \\
\hline Ulnar artery $(\mathrm{d})$ & $2.0(0.6)$ & $1.7(0.5)$ & $<0.001^{*}$ \\
\hline Ulnar artery $(q)$ & $11(8)$ & $7(7)$ & 0.135 \\
\hline Cephalic vein & $2.4(1.0)$ & $2.4(0.8)$ & 0.940 \\
\hline \multicolumn{4}{|l|}{ AVF Configuration ${ }^{\#}$} \\
\hline RCAVF & $64(40)$ & $21(22)$ & \\
\hline BCAVF & $51(32)$ & $29(31)$ & \\
\hline BBAVF & $41(26)$ & $44(46)$ & \\
\hline Brachiocubital $^{\$}$ & $3(2)$ & $1(1)$ & \\
\hline
\end{tabular}

Venous diameter measurements are in [mm], arterial diameters (d) also in [mm], and arterial volume flow (q) in $[\mathrm{mL} / \mathrm{min}]$. All measurements are performed halfway the upper respectively forearm. Values are reported as means (SD) for upper arm and forearm measurement, and $\mathrm{n}$ (\%) for AVF configuration. * denotes a statistically significant difference at the 0.05 significance level using an independent t-test. \# statistically significant difference. For Table 3a the Chi-square $=41.149, \mathrm{p}<0.001$, albeit that the minimum expected count is 1.22 , for Table $3 \mathrm{~b}$ these are respectively $13.354, \mathrm{p}<0.001$, and 30.08 . $\$$ not included in any of the further analyses. 
Table 4 Postoperative changes directly - 3 months.

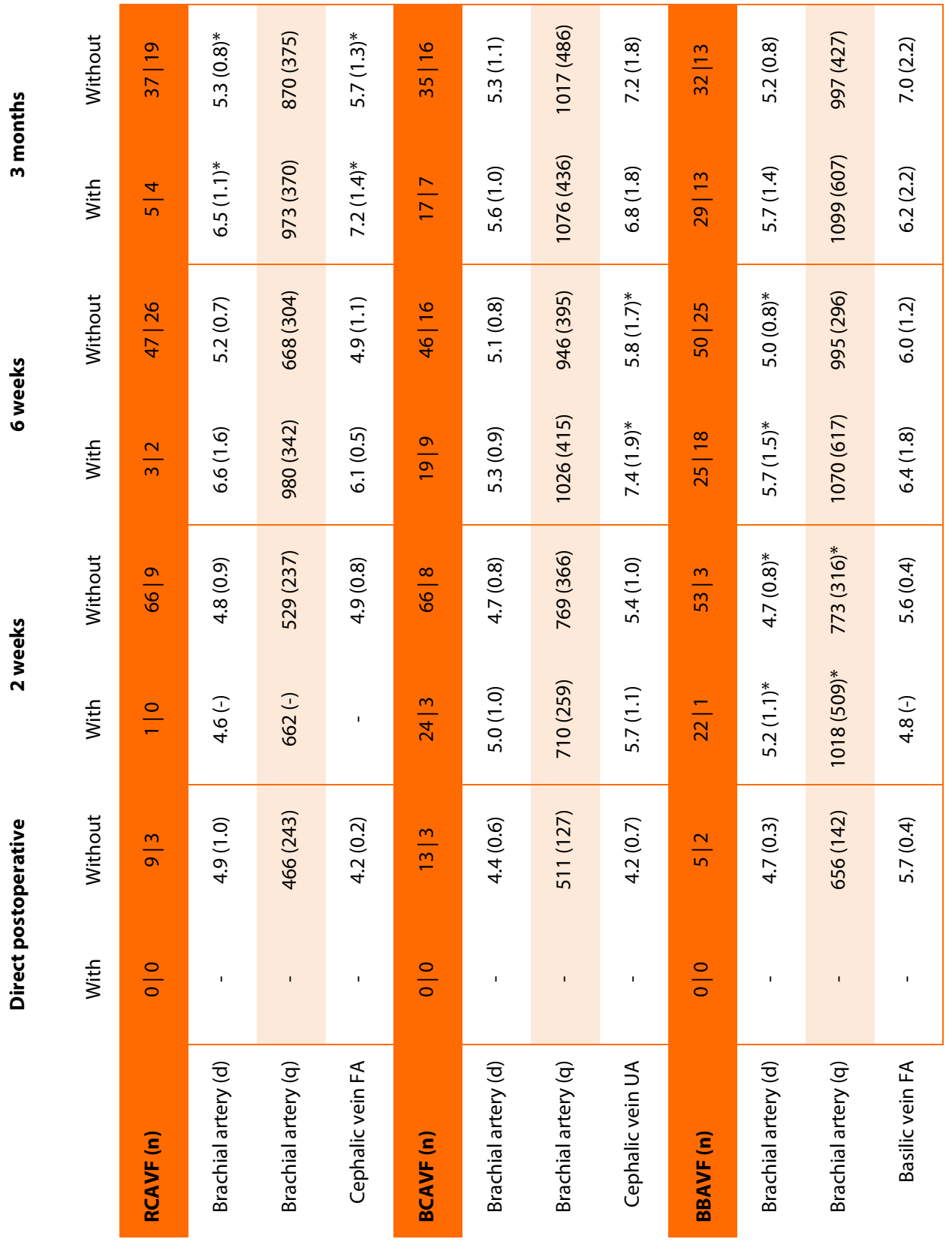

Venous measurements are in [mm], arterial diameters (d) also in [mm], and arterial volume flow (q) in [mL/min]. Reported values are means (SD). All measurements are performed halfway the upper- or forearm (UA respectively FA). * denotes a statistically significant difference at the 0.05 significance level using independent t-tests. The " $n \mid n$ " in the configuration row denotes the number of arterial and venous measurements available for the respective follow-up moments for that configuration. 
During follow up, the preoperatively found differences in arterial and venous diameters gradually disappeared or at least were less consistent (Table 4). At two weeks follow up the brachial artery diameter and flow for the BBAVF were significantly greater in patients with a previous VA (5.2 vs. $4.7 \mathrm{~mm} ; \mathrm{p}=0.015$, and 1018 vs. $773 \mathrm{~mL} / \mathrm{min} ; \mathrm{p}=0.013$ ); this difference diminished at 6 weeks (5.9 vs. $5.0 \mathrm{~mm} ; \mathrm{p}=0.029$, and 1070 vs. $995 \mathrm{~mL} / \mathrm{min}$; $\mathrm{p}=0.556$ ) and disappeared at 3 months of follow up (5.7 vs $5.2 \mathrm{~mm} ; \mathrm{p}=0.074$, and 1099 vs. $997 \mathrm{~mL} / \mathrm{min} ; \mathrm{p}=0.449$ ). Patients with $B C A V F s$ showed a statistically significant difference for the cephalic vein diameter at 6 weeks postoperatively ( 7.4 vs. $5.8 \mathrm{~mm} ; \mathrm{p}=0.041$ ).

Table 5 shows the percentages of AVFs that comply with the UAB and Ro6s maturation criteria. According to the UAB criteria $50 \%$ of the AVFs were already mature direct postoperatively, and reached a plateau around $85 \%$ by two weeks. Conversely, only $13 \%$ of the AVFs were mature direct postoperative according to the Ro6s, and this number continued to increase during the three postoperative months to $55 \%$.

Table 5 Percentages of arteriovenous fistulae (AVFs) that meet the University of Alabama at Birmingham (UAB) respectively Rule of $6 \mathrm{~s}$ criteria for duplex ultrasonography based maturation.

Direct PO $(n=8) \quad 2$ weeks $(n=24) \quad 6$ weeks $(n=96) \quad 3$ months $(n=71)$

\section{Results present study}

\begin{tabular}{r|rrrr} 
UAB criteria & 50 & 88 & 83 & 83 \\
Rule of $6 \mathrm{~s}$ & 13 & 21 & 37 & 55 \\
\hline Results Robbin et al. & 11 & & & - \\
\hline UAB criteria & 54 & 71 & 70 & - \\
Rule of $6 \mathrm{~s}$ & 12 & 35 & 45 & \\
\hline
\end{tabular}

Percentages of Robbin et al. are composite maturation rates of 602 AVFs for both forearm and upper arm (Table 5 of said publication). ${ }^{11}$ This study was limited to the first six weeks postoperatively, and used multiple imputation for missing values of AVF vein or flow. UAB criteria are an AVF vein diameter $\geq 4 \mathrm{~mm}$, and an AVF flow $\geq 500 \mathrm{~mL} / \mathrm{min}$. ${ }^{9}$ For the Rule of $6 \mathrm{~s}$ these values are $\geq 6 \mathrm{~mm}$, and $\geq 600 \mathrm{~mL} / \mathrm{min}$. ${ }^{6}$

In general, for all three configurations with and without a previous VA, the steepest increase in flow occurred in the first few postoperative weeks, and reached a plateau by three months postoperatively (Figures $1 \mathrm{a}-1 \mathrm{c}$ ). The flow in RCAVFs progressed from $466 \pm 243 \mathrm{~mL} / \mathrm{min}$ direct postoperatively, to $530 \pm 236$ by two weeks $(p=0.008), 687 \pm 312$ at six weeks $(p=0.003)$, and $882 \pm 371 \mathrm{~mL} / \mathrm{min}$ by three months $(p=0.158)$. For BCAVFs the respective AVF flows were $511 \pm 124,753 \pm 340(p=0.021), 970 \pm 400(p<0.001)$, and $1023 \pm 486 \mathrm{~mL} / \mathrm{min}(p=0.151)$, whereas the flow development for BBAVFs was $656 \pm 142$, $845 \pm 396(p=0.197), 1021 \pm 434(p=0.007)$, and $1046 \pm 518 \mathrm{~mL} / \mathrm{min}(p=0.434)$. Assessing the flow development by group showed that patients without a previous ipsilateral VA 
already reached a plateau between 2 and 6 weeks, whereas for patients without this was between 6 weeks and 3 months. For RCAVFs there was too few data to make a comparison, and for BCAVFs there was no difference (both between 6 weeks and 3 months). The direct postoperative AVF flow of the three AVF configurations is comparable, whereas at 2 and 6 week follow up there is a significant difference in flow, which disappeared at 3 months follow up. Table 4 and Figure 1 show the results until 3 months postoperatively. Results of hemodynamical changes up to 2 years can be found in supplementary Table S1.

Figure 1a-c Boxplots of radiocephalic (A), brachiocephalic (B), and brachiobasilic (C) arteriovenous fistula development during the first three months postoperatively. Left panels are the venous diameters $[\mathrm{mm}]$, and in the right panels brachial artery flow $[\mathrm{mL} / \mathrm{min}]$. Diameter and flow for patients without a previous vascular access are on the left (in blue), with a previous vascular access on the right (in orange). Solid squares denote measurement means, crosses are outliers. AVF: arteriovenous fistula; PO: postoperative; FU: follow-up. (Continues on the next page.)

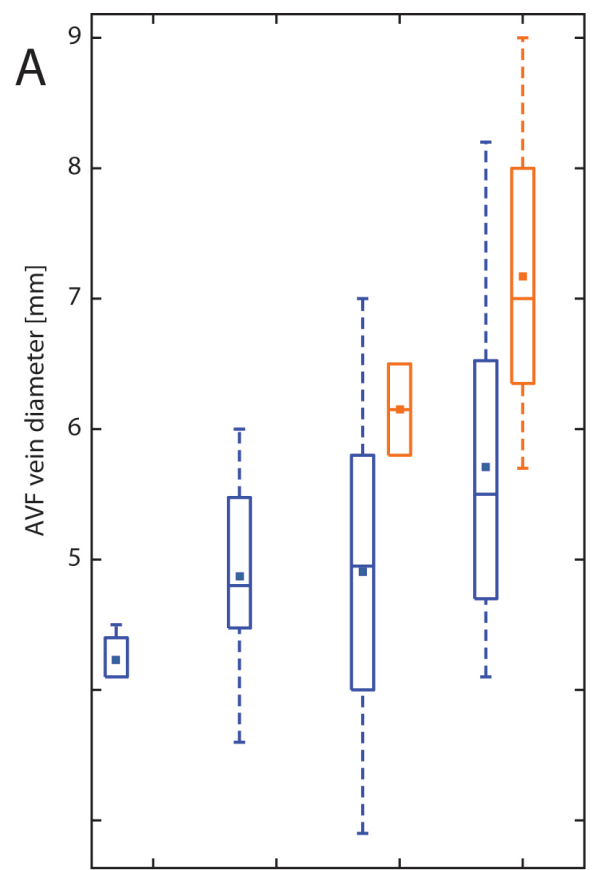

Direct PO Two weeks Six weeks Three months FU Moment

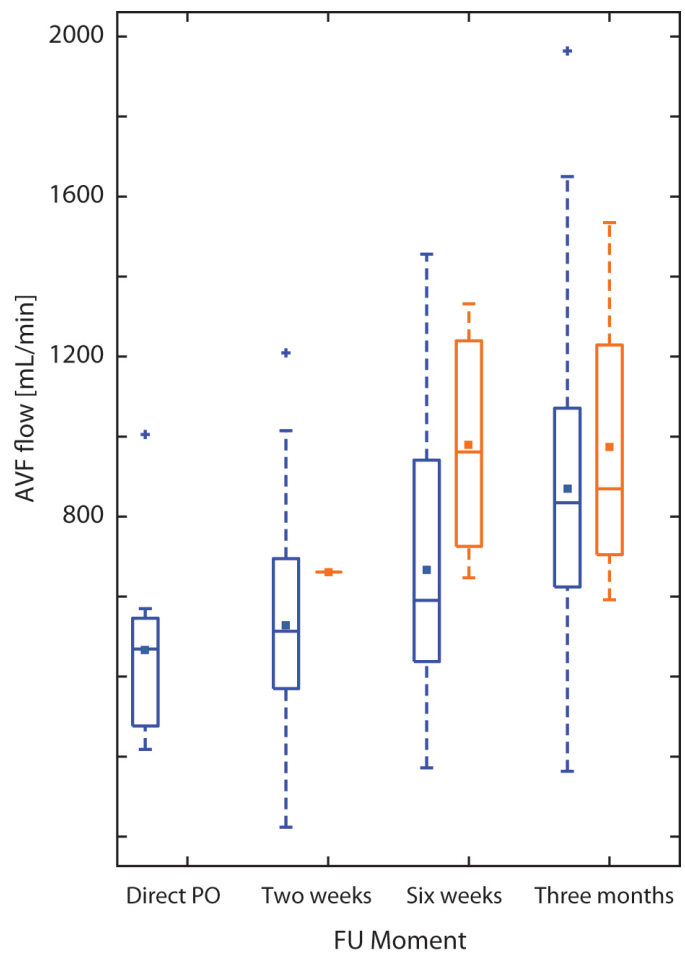



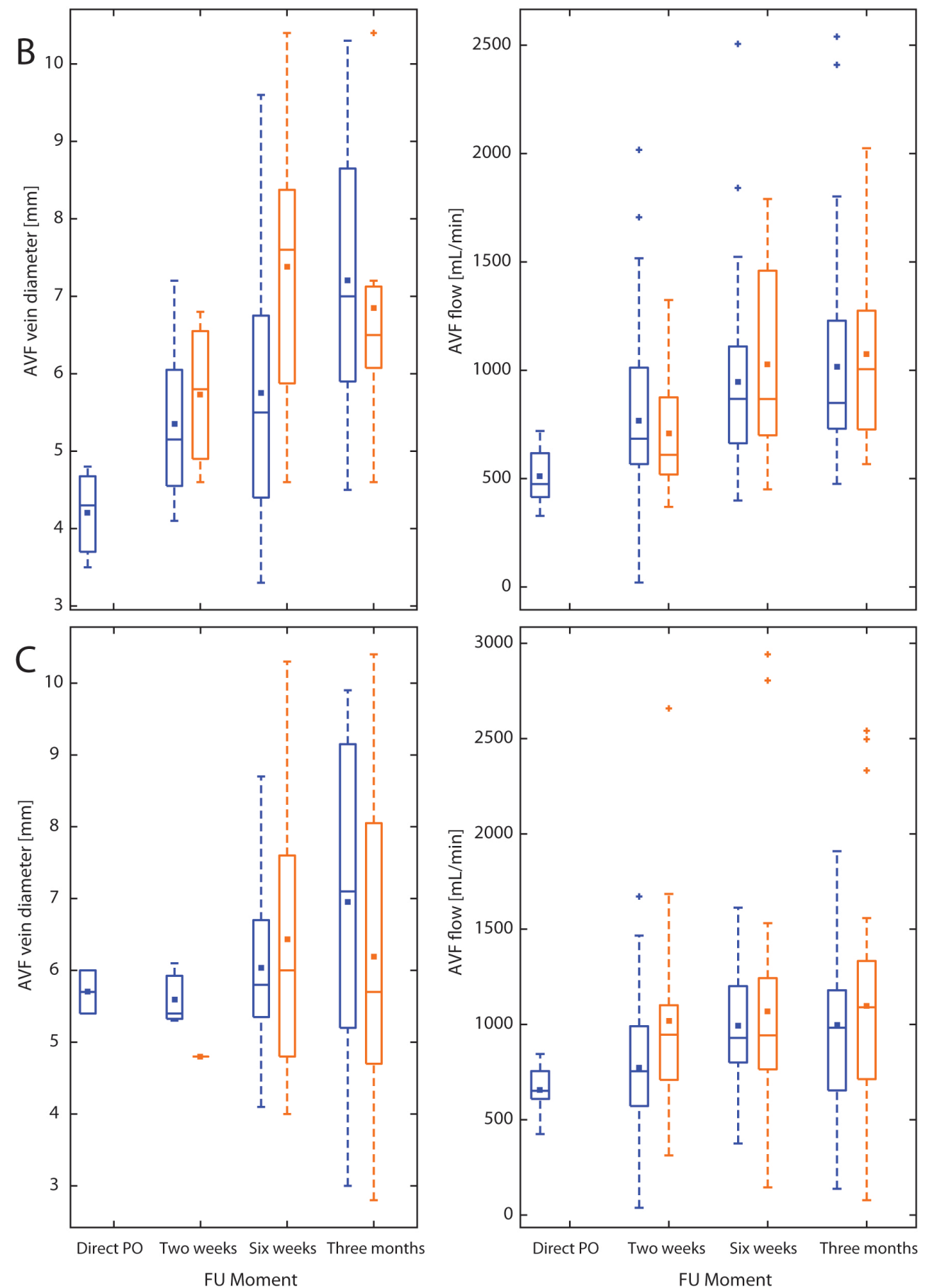

FU Moment

FU Moment 


\section{DISCUSSION}

Little is known about the hemodynamical changes after AVF creation beyond six weeks postoperatively, and how these changes may or may not be affected by any previous ipsilateral VA. The main finding of the present retrospective study is that the progressive changes in AVF vein and flow continue beyond the often studied six weeks postoperatively. In patients with a previous ipsilateral VA the data suggests that the maturation process might accelerate, in comparison to patients without a previous ipsilateral VA in whom the hemodynamically changes can occur up to 3 months postoperatively.

The observed changes are a remodeling response of the blood vessel induced by the endothelium. Endothelial cells sense high wall shear stress (WSS) and consequently trigger a signaling cascade that aims to normalize these high WSS values. ${ }^{16}$ It has been shown in patients that received an RCAVF that mean WSS acutely increases, and that the radial and brachial artery in response increases their vessel diameters to lower the mean WSS. ${ }^{14,17}$ The radial artery already reached a plateau at three weeks, whereas the brachial artery continued to remodel up to one year postoperatively. ${ }^{14}$ The latter finding is also observed in our data (Table S1). Another study found comparable results during the first three months, and consequently concluded that presumably the peak WSS rather than the mean WSS is the driving remodeling force, since the former normalizes, and the latter remains elevated in comparison to baseline values. ${ }^{18}$ Although WSSs were not studied in this study, the reported arterial diameter changes do show comparable trends.

The hemodynamical changes predominantly take place during the first two weeks, but continue up to three months for all AVF configurations; after that time they reach a plateau. The hemodynamical changes till 12 weeks after RCAVF creation ( $n=93)$, as described by Malovrh, show comparable outcomes. ${ }^{13}$ The AVF vein diameter appears to continuously enlarge up to 12 weeks postoperatively, while the AVF flow already reaches a plateau by three weeks, with a measured flow of $400-450 \mathrm{~mL} / \mathrm{min}^{13}, 19,20$ Presumably, the difference in AVF flow development is due to the fact that Malovrh measured radial artery flows instead of the brachial artery flow as was done in the present study. Retrograde radial artery flow distal from the anastomosis is usually observed in patients with an RCAVF (i.e. $80 \%$ ), and contributes for $25 \%$ of the AVF flow. ${ }^{21,22}$ Robbin et. al. have published two studies on the same issue with conflicting results., 11 In the first study they concluded that there is no change in AVF vein diameter (stable from 4.0 to $4.5 \mathrm{~mm}$ ) or flow (stable from 700 to $800 \mathrm{~mL} / \mathrm{min}$ ) at three postoperative time points (i.e. 2, 3, and 4 months; $n=69$ patients). This inconsistent 
finding might be explained by the fact that forearm ( $n=48 ; 70 \%)$ and upper arm AVFs $(21 ; 30 \%)$ were not separately assessed, thereby introducing large dispersion in the data, and consequently actual differences are obscured. ${ }^{9}$ In their second study, based on data from the Hemodialysis Fistula Maturation study (602 patients), they report findings that are very consistent with the data in the present study. ${ }^{11}$ The major changes in AVF vein diameter and flow occur direct postoperatively, after which this process continues to 6 weeks postoperatively. The postoperative AVF vein diameters increased from $4.0 \mathrm{~mm}$ (forearm AVF) and 5.0mm (upper arm AVF) to 5.0 and $6.5 \mathrm{~mm}$ respectively at 6 weeks postoperatively. Likewise, AVF flow increased from 400 and 750 to 600 and $1100 \mathrm{~mL} / \mathrm{min}$ respectively. ${ }^{11}$

There appears to be some sort of vessel preconditioning due to the previous ipsilateral VAs. In particular, patients had greater basilic vein diameters and higher brachial artery flows in comparison to patients without a previous access. In addition, the ulnar artery flow was significantly greater, indicating enhanced collateral flow in patients with an RCAVF. ${ }^{22,}{ }^{23}$ As mentioned before, retrograde radial flow distal from the anastomosis contributes on average $25 \%$ of AVF flow. There is however large variability between patients, as the contribution ranges from 19:1 to 1:1 in individual patients. ${ }^{23}$

Women showed smaller preoperative arterial diameters compared to men. Robbin et al. reported very similar results with differences in brachial and radial artery diameters for both sexes. ${ }^{11}$ The brachial artery was 3.6 vs $4.5 \mathrm{~mm}$, and the radial artery $2.1 \mathrm{vs} 2.3 \mathrm{~mm}$ for women and men respectively. Conversely, upper arm and forearm vein diameters were comparable with 3.0 and $2.4 \mathrm{~mm}$ for women, and 3.2 and $2.5 \mathrm{~mm}$ for men. BC- and BBAVFs were not separately assessed. ${ }^{11}$ A similar pattern in AVF configurations was observed. Patients with small arterial diameters may have higher resistance conduits and therefore surgeons may opt to create an upper arm AVF instead of a forearm AVF, in particular in females. Although the observed pattern is comparable to those of the present study, the absolute numbers differ slightly. In the Robbin study, RCAVFs were created in $11 \%$ of the women and $29 \%$ of the men. ${ }^{11}$ In our study these numbers were 22 and $40 \%$ respectively. In literature similar observations are reported with smaller arterial diameters and fewer forearm AVFs created in women. ${ }^{24-28}$

In the present study the three AVF configurations in patients without a previous ipsilateral VA are equally distributed, which is not in agreement with other studies in which the RCAVF is the predominant configuration. ${ }^{3,8,29}$ Possibly, this equal distribution of AVF configurations is a representation of the patient population of a tertiary referral center with over $50 \%$ of access procedures performed on referred patients. Referred patients have usually a limited number of AVF sites available, even without a previous ipsilateral VA. And in the group of patients with a previous ipsilateral VA, the BBAVF was the predominant configuration. 
There is no obvious benefit of vessel preconditioning with accelerated fistula maturation observed in patients with a previous ipsilateral VA. One might expect a lower FTM rate or an increased ischemia/high flow risk. This lack of benefit or disadvantage of vessel preconditioning may be due to the fact that exclusively the natural flow development was assessed, and patients with VA interventions were excluded for analysis.

The present study might warrant reconsidering the length of the maturation time. This position is substantiated by the recent study by Wilmink et al. that described the natural history of autologous fistulae in terms of functionality. ${ }^{8}$ They found that the median time for an AVF to become functional ranges from 9.4 to 13.4 weeks for BC- and BBAVFs respectively. ${ }^{8}$ Although their definition of AVF maturation included at least six successful dialysis sessions, it clearly shows that AVFs require more than six weeks to mature. They argue that a RCAVF should be created at least 4 months before the anticipated dialysis start. The present study supports this position in the sense that by three months the hemodynamical changes reached a plateau, and the AVF seems to be maximally matured. In addition, our data supports the suggestion by Lee et al. to reconsider the venous diameter and arterial flow criteria of the Rule of $6 \mathrm{~s}$, and rather use the University of Alabama at Birmingham (UAB) criteria of $4 \mathrm{~mm}$ respectively $500 \mathrm{~mL} / \mathrm{min} .{ }^{30}$ At six weeks and three months the AVF vein diameters are in the range of $6 \mathrm{~mm}$ (Table 4), with standard deviations greater then $1 \mathrm{~mm}$. Table 5 presents a direct comparison between both criteria sets. Eighty-eight percent of the AVFs already comply with the UAB criteria at 2 weeks postoperatively, but more importantly this percentage remains relatively stable during the next 3 months (83\%). The data for the Rule of $6 \mathrm{~s}$ show a continuous increase of flow and diameter, starting with only $21 \%$ of the AVFs that meet the criteria at 2 weeks and $55 \%$ at 6 weeks.

The present study has several strengths. First, the changes observed during the first six weeks are in concordance with previous reports, indicating a representative study with readily generalizable findings. Second, it is a large single center study that describes the natural AVF development beyond the usually taken period of 6 weeks postoperatively in an unselected group of patients. Although these six weeks may be the most important period for maturation, it is now clear that one can expect changes up to three months postoperatively too. Third, it provides data on the effect of any previous ipsilateral VAs on the vascular anatomy, and how this in turn can affect postoperative changes. In light of a worldwide increase in prevalent HD patients this is relevant information, since patients depend longer on HD, and therewith may be in need of multiple VAs. ${ }^{31,32}$ Fourth, in this study the two upper arm AVF configurations are reported separately. Data on development of upper arm AVFs is scarce, and the present study describes the changes for two configurations without obscuring any possible differences between both, be it anatomically or surgically. 
There are also some limitations to the present study. Since the natural AVF development was studied, it lacks functional (i.e. dialysis) or clinical information in detail other than the reasons for follow-up ending. Although there appears to be no difference in primary failure or high flow complications, it remains unclear if the latter may occur more often in patients with a previous ipsilateral VA on long-term follow up. The second limitation is that the present study is situated in a tertiary referral center. In patients with a previous ipsilateral VA, the data on natural AVF development may be missing at our institution. Possibly the distribution of AVF configurations in the group without a previous VA is also negatively affected (i.e. fewer RCAVFs than reported in literature) by externally referred patients.

In conclusion, the present study shows that AVF remodeling continues beyond six weeks postoperatively, and reaches a plateau by three months. Furthermore, there appears to be a vessel preconditioning effect after a previous ipsilateral VA, resulting in an accelerated maturation but without any clear beneficial or detrimental consequences. The findings of the present study warrant reconsidering the expected/acceptable length of the maturation period. 


\section{REFERENCES}

1. Al-Jaishi AA, Oliver MJ, Thomas SM, et al. Patency rates of the arteriovenous fistula for hemodialysis: a systematic review and meta-analysis. American journal of kidney diseases. 2014;63(3):464-78.

2. Huijbregts $\mathrm{HJ}$, Bots $\mathrm{ML}$, Wittens $\mathrm{CH}$, et al. Hemodialysis arteriovenous fistula patency revisited: results of a prospective, multicenter initiative. Clinical journal of the American Society of Nephrology. 2008;3(3):714-9.

3. Masengu A, Maxwell AP, Hanko JB. Investigating clinical predictors of arteriovenous fistula functional patency in a European cohort. Clinical kidney journal. 2016;9(1):142-7.

4. Tordoir JH, Dammers R, van der Sande FM. Upper extremity ischemia and hemodialysis vascular access. European journal of vascular and endovascular surgery. 2004;27(1):1-5.

5. Scheltinga MR, van Hoek F, Bruijninckx CMA. Time of onset in haemodialysis accessinduced distal ischaemia (HAIDI) is related to the access type. Nephrology Dialysis Transplantation. 2009;24(10):3198-204.

6. National Kidney Foundation Kidney Disease Outcomes Quality Initiative: clinical practice guidelines for vascular access, update 2006. American journal of kidney diseases. 2006;48(Suppl 1):S176-247.

7. Schmidli J, Widmer MK, Basile C, et al. Vascular Access: 2018 Clinical Practice Guidelines of the European Society for Vascular Surgery (ESVS). European Journal of Vascular and Endovascular Surgery. 2018;55(6):757-818.

8. Wilmink T, Hollingworth $\mathrm{L}$, Powers $\mathrm{S}$, et al. Natural History of Common Autologous Arteriovenous Fistulae: Consequences for Planning of Dialysis Access. European Journal of Vascular and Endovascular Surgery. 2016;51(1):134-40.

9. Robbin ML, Chamberlain NE, Lockhart ME, et al. Hemodialysis arteriovenous fistula maturity: US evaluation. Radiology. 2002;225(1):59-64.

10. Shemesh D, Goldin I, Berelowitz D, et al. Blood flow volume changes in the maturing arteriovenous access for hemodialysis. Ultrasound in medicine $\&$ biology. 2007;33(5):727-33.

11. Robbin ML, Greene T, Cheung AK, et al. Arteriovenous Fistula Development in the First 6 Weeks after Creation. Radiology. 2016;279(2):620-9.

12. Tordoir $\mathrm{JH}$, Rooyens $\mathrm{P}$, Dammers $\mathrm{R}$, et al. Prospective evaluation of failure modes in autogenous radiocephalic wrist access for haemodialysis. Nephrology, dialysis, transplantation. 2003;18(2):378-83.

13. Malovrh M. Postoperative assessment of vascular access. The journal of vascular access. 2014;15 Suppl 7:S10-4.

14. Dammers R, Tordoir JH, Kooman JP, et al. The effect of flow changes on the arterial system proximal to an arteriovenous fistula for hemodialysis. Ultrasound in medicine \& biology. 2005;31(10):1327-33.

15. Zonnebeld N, Huberts W, van Loon MM, et al. Preoperative computer simulation for planning of vascular access surgery in hemodialysis patients. The journal of vascular access. 2017;18(Suppl. 1):118-24. 
16. Remuzzi A, Bozzetto $M$, Brambilla P. Is shear stress the key factor for AVF maturation? The journal of vascular access. 2017;18(Suppl. 1):10-4.

17. Dammers R, Tordoir JH, Welten RJ, et al. The effect of chronic flow changes on brachial artery diameter and shear stress in arteriovenous fistulas for hemodialysis. The International journal of artificial organs. 2002;25(2):124-8.

18. Ene-lordache B, Mosconi L, Antiga L, et al. Radial artery remodeling in response to shear stress increase within arteriovenous fistula for hemodialysis access. Endothelium. 2003;10(2):95-102.

19. Malovrh M. Non-invasive evaluation of vessels by duplex sonography prior to construction of arteriovenous fistulas for haemodialysis. Nephrology, dialysis, transplantation. 1998;13(1):125-9.

20. Malovrh M. Native arteriovenous fistula: preoperative evaluation. American journal of kidney diseases. 2002;39(6):1218-25.

21. Sivanesan S, How TV, Black RA, et al. Flow patterns in the radiocephalic arteriovenous fistula: an in vitro study. Journal of biomechanics. 1999;32(9):915-25.

22. Duncan $\mathrm{H}$, Ferguson L, Faris I. Incidence of the radial steal syndrome in patients with Brescia fistula for hemodialysis: its clinical significance. Journal of vascular surgery. 1986;4(2):144-7.

23. Sivanesan S, How TV, Bakran A. Characterizing flow distributions in AV fistulae for haemodialysis access. Nephrology, dialysis, transplantation. 1998;13(12):3108-10.

24. Allon M, Lockhart ME, Lilly RZ, et al. Effect of preoperative sonographic mapping on vascular access outcomes in hemodialysis patients. Kidney international. 2001;60(5):2013-20.

25. Miller CD, Robbin ML, Allon M. Gender differences in outcomes of arteriovenous fistulas in hemodialysis patients. Kidney international. 2003;63(1):346-52.

26. Lockhart ME, Robbin ML, Allon M. Preoperative sonographic radial artery evaluation and correlation with subsequent radiocephalic fistula outcome. Journal of ultrasound in medicine. 2004;23(2):161-8; quiz 9-71.

27. Korten E, Toonder IM, Schrama YC, et al. Dialysis fistulae patency and preoperative diameter ultrasound measurements. European journal of vascular and endovascular surgery. 2007;33(4):467-71.

28. Peterson WJ, Barker J, Allon M. Disparities in fistula maturation persist despite preoperative vascular mapping. Clinical journal of the American Society of Nephrology. 2008;3(2):437-41.

29. Lok CE, Allon M, Moist $L$, et al. Risk equation determining unsuccessful cannulation events and failure to maturation in arteriovenous fistulas (REDUCE FTM I). Journal of the American Society of Nephrology. 2006;17(11):3204-12.

30. Lee T, Magill M, Burke SK, et al. Comparison of postoperative ultrasound criteria to predict unassisted use of arteriovenous fistulas for hemodialysis. The journal of vascular access. 2018;19(2):167-71.

31. Kramer A, Pippias M, Noordzij M, et al. The European Renal Association - European Dialysis and Transplant Association (ERA-EDTA) Registry Annual Report 2015: a summary. Clinical kidney journal. 2018;11(1):108-22. 
32. System USRD. USRDS annual data report: An overview of the epidemiology of kidney disease in the United States. 2017; Volume Two: End-Stage Renal Disease in the United States. 
Table S1 Postoperative changes 6 months - 2 years.

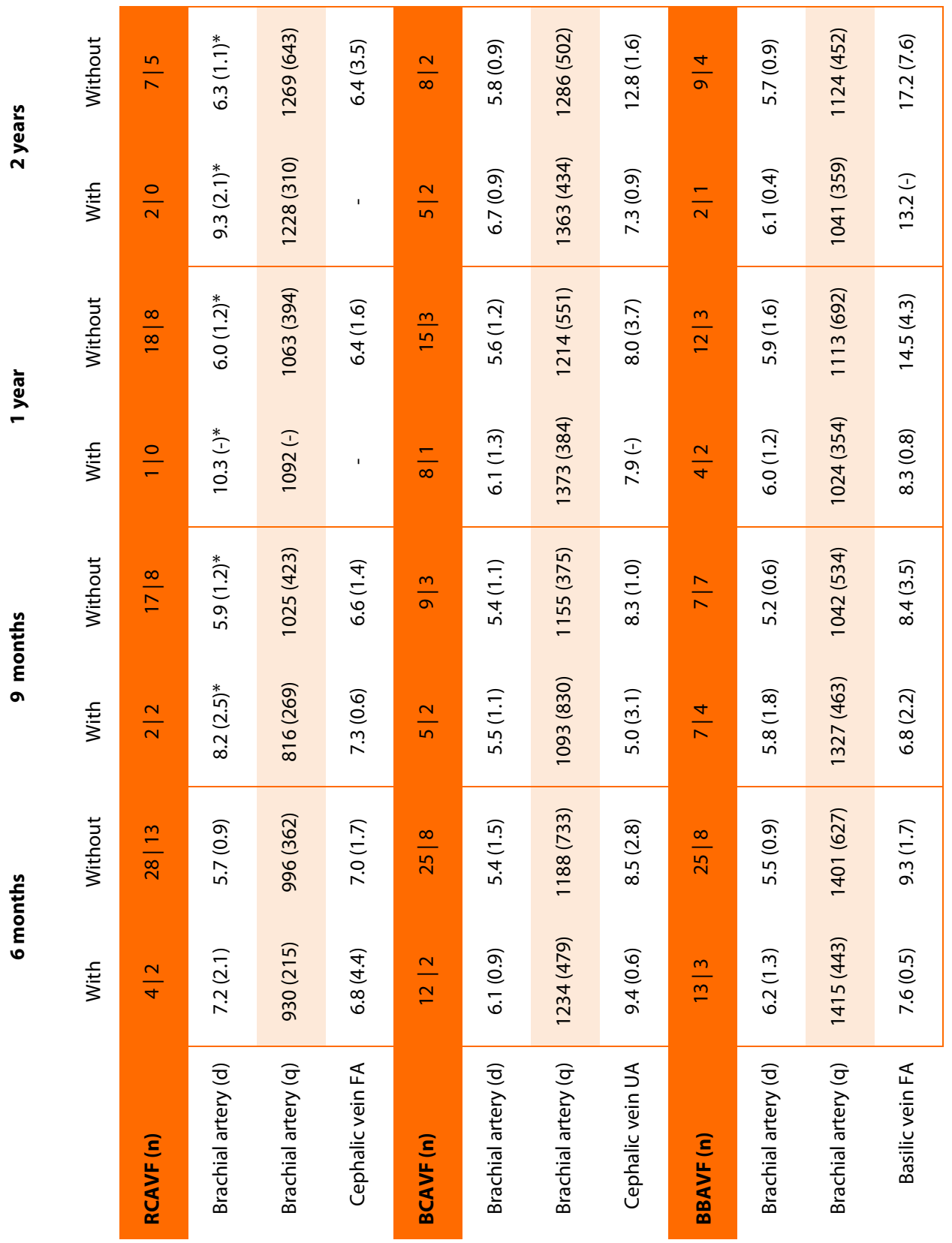

Venous measurements are in [mm], arterial diameters (d) also in [mm], and arterial volume flow (q) in [mL/min]. Reported values are means (SD). All measurements are performed halfway the upper- or forearm (UA respectively FA). * denotes a statistically significant difference at the 0.05 significance level using independent t-tests. The " $n \mid n$ " in the configuration row denotes the number of arterial and venous measurements available for the respective follow-up moments for that configuration. 
P

a

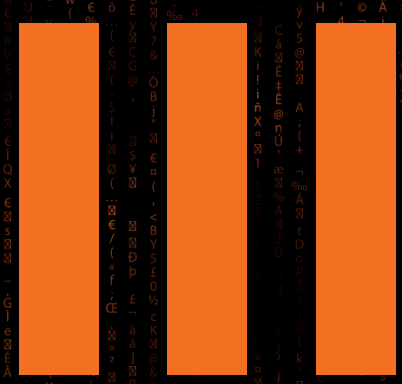

$I$

t 
ASSESSING RESULTS

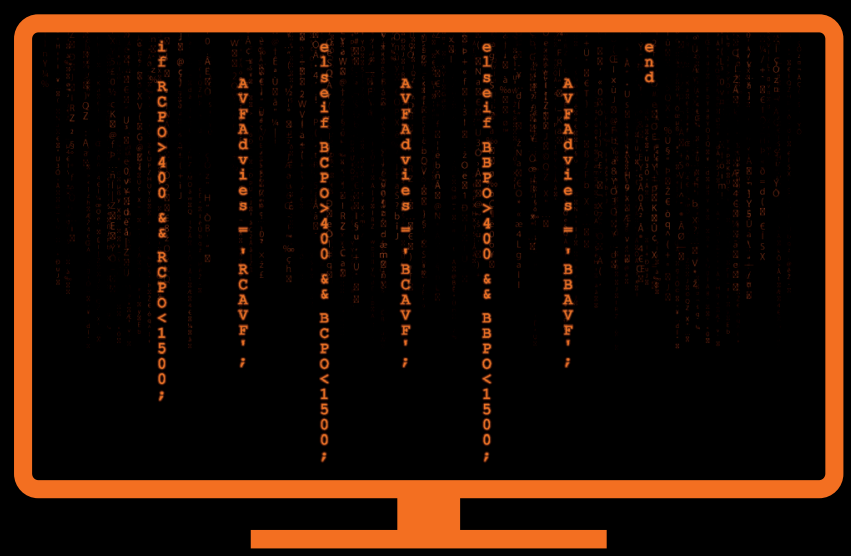





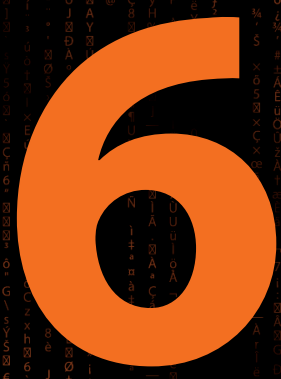

Preoperative Patient-specific Flôw Predictions to Improve Hemodialysis Arteriovenous Fistula Maturation (Shunt Simulation Study): a Randómized Controlled Trial

Niek Zonnebeld, ${ }^{1,2}$ Jan H.M. Tordoir, ${ }^{2}$ Magda M. van Loon, ${ }^{2}$ André A.E.A. de Smet, ${ }^{3}$ Laûrêns C. Huisman, ${ }^{4}$ Philippe W.M. Cuypers, ${ }^{5}$ Felix J.V. Schlössere, Susan Lemson, ${ }^{7}$ Stefan G.H. Heinen, ${ }^{8}$ Lee H. Bouwman, ${ }^{9}$ Raechel J. Toorop, ${ }^{10}$ Wouter Huberts, ${ }^{1}$ Tammo Delhaas, ${ }^{1}$ on behalf of the Shunt Simulation Study group.

Submitted

1. Department of Biomedical Engineering, Maastricht University, Maastricht, The Netherlands 2. Department of Vascular Surgery, Maastricht University Medical Center, Maastricht, The Netherlands 3. Department of Surgery, Maasstadhospital, Rotterdam, The

4. Department of Surgery, Flevoziekenhuis, Almere, The Netherlands

5. Department of Surgery, Catharina hospital, Eindhoven, The Netherlands

6. Department of Surgery, Laurentius hospital, Roermond, The Netherlands

7. Department of Surgery, Slingeland hospital, Doetinchem, The Netherlands

8. Department of Surgery, St. Antonius hospital, Nieuwegein, The Netherlands

9. Department of Surgery, Zuyderland Medical Center, Heerlen, The Netherlands 10. Department of Surgery, University Medical Center Utrecht, Utrecht, The Netherlands 


\section{ABSTRACT}

An arteriovenous fistula (AVF) needs to mature before it becomes usable for hemodialysis. AVF maturation importantly depends on the postoperative flow increase. Unfortunately, 20-40\% of created AVFs fail to mature (FTM). A patient-specific computational model that predicts immediate postoperative flow was previously developed, and it was hypothesized that having this additional information available during surgical planning can reduce FTM rates.

\section{Methods}

A multicenter randomized controlled trial in nine Dutch hospitals was conducted in which (pre)hemodialysis patients that were referred for AVF creation were recruited. Patients were randomly assigned (1:1) for the control or simulation group. Both groups underwent an extensive work-up, including duplex ultrasonography (DUS) examination. In the simulation group the data from the DUS examination were used for model simulations and based on the immediate postoperative flow prediction the ideal AVF configuration was recommended. Primary endpoint was FTM defined as an AVF flow $<500 \mathrm{~mL} / \mathrm{min}$ and/or vein diameter $<4 \mathrm{~mm}$ at six weeks postoperatively. Secondary endpoint was model performance (i.e. comparisons measured and predicted flows, and (multivariate) regression analysis for maturation likelihood with accompanying area under the receiver operator characteristic curve [AUC]).

\section{Findings}

236 patients were randomly assigned (116 in the control group and 120 in the simulation group), of which 205 (100 respectively 105) were analyzed for the primary endpoint. There was no difference in FTM rates with 29 respectively $32 \%$. Immediate postoperative flow prediction had an OR of $1.15(1.06-1.26 ; p<0.001)$ per $100 \mathrm{~mL} / \mathrm{min}$ for maturation, and the accompanying AUC was 0.67 (0.59-0.75).

\section{Interpretation}

Patient-specific immediate postoperative flow predictions are predictive for AVF maturation. Having this additional information available during surgical planning, however, does not (yet) result in reduced FTM rates. 


\section{INTRODUCTION}

Arteriovenous fistulae (AVFs) are the preferred vascular access for hemodialysis over arteriovenous grafts and central venous catheters. AVFs show fewer thromboses, less infections, and have superior patency rates. ${ }^{1}$ However, a prerequisite for these benefits is that the AVF matures sufficiently. Its volume flow should increase to $\geq 500 \mathrm{~mL} / \mathrm{min}$, and venous diameters should be sufficiently large $(\geq 4 \mathrm{~mm})$ to allow easy cannulation and effective dialysis at approximately six weeks postoperatively. ${ }^{2}$ Unfortunately, $20-40 \%$ of constructed AVFs fail to mature (FTM). ${ }^{3,4}$ With a worldwide persistent increase in both incidence and prevalence of patients in need of hemodialysis, FTM remains an important issue..$^{5,6}$

Previously it has been shown that identification of suitable vessels for arteriovenous anastomosis by preoperative duplex ultrasound (DUS) scanning can positively influence AVF maturation rates. ${ }^{7}$ However, despite this preoperative work-up, a considerable proportion of AVFs still show FTM. In an effort to further reduce FTM rates, several maturation prediction models are designed, of which the Risk Equation Determining Unsuccessful Cannulation Events and Failure to Maturation (i.e. REDUCEFTM) score is probably the most well-known and studied model. ${ }^{8}{ }^{9}$ Unfortunately, as these prediction models are predominantly multivariable regression models, they are not truly patient-specific, and their generalizability strongly depends on comparability of the dialysis population on which they are designed and applied as they do not incorporate mechanistical relations. ${ }^{4,10}$

Recently, a patient-specific postoperative flow prediction model (based on physical laws) was developed. ${ }^{11}$ Since flow is an important mediator of maturation it was hypothesized that providing postoperative flow predictions in surgical planning can positively influence maturation rates. The most likely to mature AVF configuration can then be preoperatively recommended with this tool. The computational model was found in a previous study to accurately predict immediate postoperative blood flow volume based on individual preoperative diameter and flow measurements attained by DUS. ${ }^{12,13}$ To date it is not proven whether these predictions also contribute to lower FTM rates when provided as additional preoperative information to the surgeon. The present study reports on the results of a randomized clinical trial which aims to assess the effect of this clinical application on FTM rates. ${ }^{14}$ 


\section{METHODS}

\section{Trial design}

An unblinded multicenter randomized clinical trial was performed in nine Dutch hospitals with medium to large dialysis facilities (100-150 chronic dialysis patients). The allocation ratio was 1:1, with block randomization per center. The trial was approved by the institutional review board of Maastricht UMC+. The present article is drafted in concordance with the Consolidated Standards of Reporting Trials for Nonpharmacologic Treatments (i.e. CONSORT NPT). ${ }^{15}$

\section{Participants}

Patients in need for a vascular access for chronic hemodialysis treatment and aged 18 years and older, were eligible for participation if the following inclusion criteria were met: an autogenous AVF is deemed possible by the treating physician, and the patient can provide written informed consent. Exclusion criteria were a previous ipsilateral vascular access or contra-indications for an AVF.

\section{Interventions}

The intervention under investigation is a patient-specific physics-based computational model that provides additional preoperative information by predicting postoperative flows. This computational model has been described in detail elsewhere. ${ }^{11}$ In short, it is a pulse wave propagation model which is based on mass and momentum balance laws, and describes how pressure and flow pulses travel along the arterial tree. The relevant part of the arterial tree is divided in several serially connected segments that describe the local relation between pressure and flow via physical parameters as resistance, compliance, and inertia. The parameters of each individual segment can be made patient-specific by input of volume flows and diameters, measured by DUS at the corresponding anatomical locations, into the model. This model has been shown to reliably predict postoperative blood flow volumes. ${ }^{12}{ }^{13}$ This prediction is done for the three main AVF configurations (i.e. radiocephalic [RC-], brachiocephalic [BC-], and brachiobasilic AVF [BBAVF]). The most distal configuration that meets the literaturebased threshold of $\geq 400 \mathrm{~mL} / \mathrm{min}$, as predicted by the model is recommended. ${ }^{16}$ Figure 1 provides a schematic representation of recommendation generation. 
INPUT

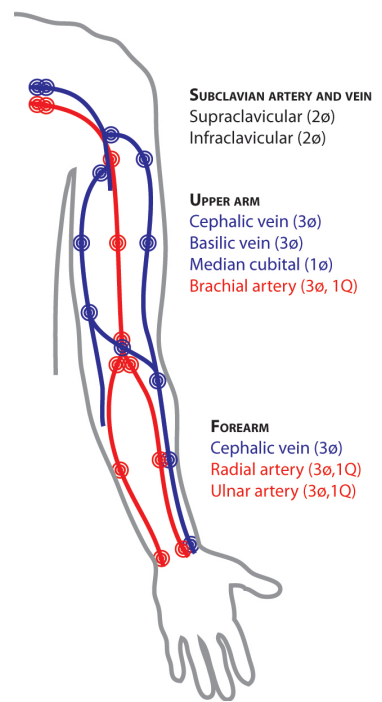

MODEL

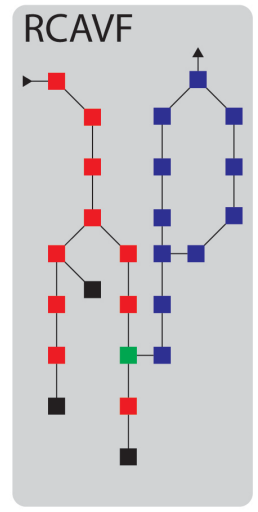

Patient-specific DUS measurement locations (model input) (ㄱ) arterial (øand Q) () venous (ø)

Corresponding PWPM elements
arterial
venous Other PWPM elements anastomosis peripheral resistance

\section{OUTPUT}
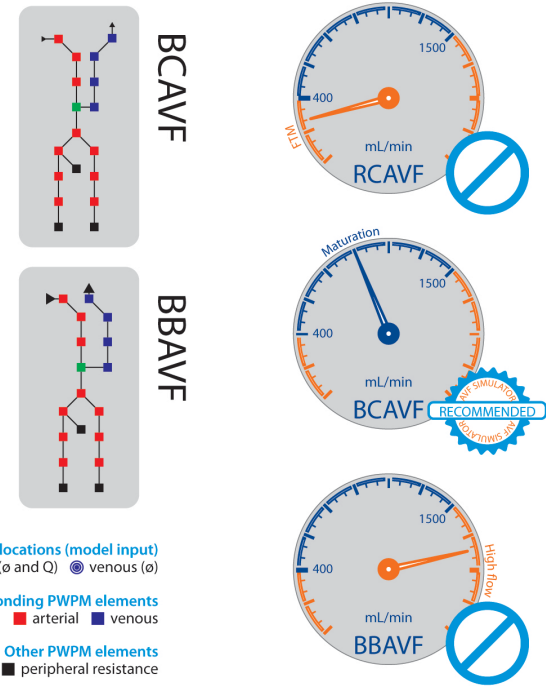

Figure 1 Schematic representation of a simulation and recommendation example. In the left panel the various measurement locations are represented schematically. For a detailed description see Zonnebeld et al. ${ }^{37}$ In the middle panel the schematic representation of the various arteriovenous fistula (AVF) configurations in the pulse wave propagation model (PWPM) is shown. Each red and blue square represents the corresponding anatomical location, and locally describes the relation between pressure and flow using parameters as resistance, compliance, and inertia. The radiocephalic (RC-), brachiocephalic (BC-), and brachiobasilic (BBAVF) configurations are simulated and the resultant predicted flows compared with predefined cut-off values. The most distal configuration with a flow between 400 and $1500 \mathrm{~mL} / \mathrm{min}$ will be recommended (BCAVF in this example). Ø: diameter measurement; Q: flow measurement; DUS: duplex ultrasonography.

The pre- and postoperative DUS examinations were standardized in the participating hospitals. The preoperative measurement protocol ${ }^{14}$ was based on the minimum set of input data necessary for model simulations, which means that at 12 discrete locations the venous diameter, and at 11 discrete locations the arterial diameter, including blood volume flow was measured (Figure 1). The postoperative DUS examination focused on the arterial inflow, anastomosis and outflow vein of the AVF conduit. Direct postoperatively (i.e. until one week) a brachial artery volume flow measurement was performed. Thereafter, the complete AVF conduit was assessed at the same distinct preoperative locations at 6 weeks, 6 months, and 12 months. All other procedures or interventions (e.g. percutaneous transluminal angioplasty [PTA]) were not standardized and left to the discretion of the treating physician to ensure the study to be representative for regular patient care. The trial design has been described in detail elsewhere. ${ }^{14}$ 


\section{Surgical arteriovenous fistula construction}

The choice of type of anesthesia was either local, regional (i.e. plexus block) or general anesthesia. All AV anastomoses were performed in an end-of-vein-to-side-of-artery fashion. In general, the surgical procedures for the various configurations are as follows. The incision for the RCAVF is placed $\pm 5 \mathrm{~cm}$ proximally from the wrist, where the radial artery and cephalic vein are dissected from the surrounding tissues. Subsequently, the cephalic vein is distally transected and mobilized towards the radial artery where it is anastomosed with a running polypropylene suture (prolene 6-0) with an anastomosis length of 10 to $15 \mathrm{~mm}$. For BCAVFs the incision is made $\pm 2 \mathrm{~cm}$ distally from the elbow crease, with dissection of the cubital and cephalic vein and division of the lacertus fibrosis. Thereafter, the brachial artery is dissected and anastomosed to the distally transected vein with running prolene $6-0$ with an anastomosis length of $7 \mathrm{~mm}$. BBAVFs were either done in a one or two-staged procedure. The basilic vein is dissected and transected as distally as possible in the upper arm through a continuous or interrupted medial longitudinal incision. Then, the brachial artery is dissected through a transverse incision $2-5 \mathrm{~cm}$ proximally from the elbow crease. Next the basilic vein is pulled through an anterolateral subcutaneous tunnel and anastomosed to the brachial artery with prolene 6-0 and an anastomosis length of $7 \mathrm{~mm}$.

\section{Outcomes}

The primary endpoint of AVF maturation is assessed by DUS, and defined as a minimal volume flow of $500 \mathrm{~mL} / \mathrm{min}$ measured at the brachial artery, and a minimal venous diameter of $4 \mathrm{~mm}$ at six weeks postoperatively. ${ }^{2}$ Model performance is assessed for immediate postoperative flows, the agreement of above or below the recommendation threshold (i.e. diagnostic values), and recommendation compliance by comparing the created AVF configuration with the recommended configuration, and whether this is different from the initial planning. Patients in the control group were simulated as well for the secondary endpoints. Secondary, next to the flow predictions the influence of other known or presumed covariates on maturation is assessed (i.e. age, sex, diabetes, peripheral arterial disease, cardiovascular disease, cerebrovascular disease, hypercholesterolemia, race, regional anesthesia, and dialysis state). ${ }^{4,8,17}$

\section{Sample size}

A sample size of 236 patients was needed, based on a power calculation for independent proportions (two tailed, $a=0.05, \beta=0.80$ ), and accounting for a $10 \%$ 
patient drop-out. Based on the literature an FTM rate of 33\% in the control group was assumed, and an FTM rate reduction of $50 \%$ in the simulation group was considered as clinically relevant.

\section{Randomization}

Consecutively enrolled patients were randomly assigned to either the control or simulation group by the coordinating investigator, guaranteeing local allocation concealment. For the coordinating center, the randomization was performed by a colleague not otherwise involved in the study or patient care - again guaranteeing allocation concealment. The R-package 'blockrand' ( $\mathrm{R}$ x64 3.2.0, www.r-project.com; blockrand version 1.3) was used for per center block randomization, with block size automatically determined based on the expected inclusion numbers.

\section{Blinding}

Though this trial was not blinded bias was minimized because the primary endpoint was not assessed by the treating physicians but by vascular technicians that were neither informed on the outcome of randomization nor on any patient-specific flow predictions.

\section{Statistical methods}

Continuous variables are presented as mean \pm standard deviation or median (interquartile range) where appropriate after data exploration, and tested for significance with appropriate t-test or Mann-Whitney test respectively. Categorical variables are presented as count and percentage, and tested for significance using logistic regression analysis or Chi-square test. The primary endpoint is assessed using logistic regression analysis. The secondary endpoint of maturation predictors is assessed by univariate analysis first, and the statistically significant factors are subsequently entered in a multivariate regression analysis. Subsequently, the area under the curve (AUC) of the receiver operator characteristic (ROC) curve of the regression model is used to assess the goodness of fit of the regression model. Interpretation of the AUC is as follows: 0.5 indicates a test of no value, $0.51-0.69$ is a poor test, $0.70-0.79$ is a fair test,

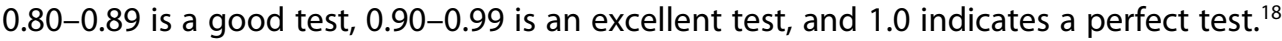
For model performance the sensitivity, specificity, positive and negative predictive values are calculated for the recommendation threshold. A p-value $<0.05$ is considered statistically significant. All statistics are performed using SPSS version 22. 


\section{RESULTS}

236 patients were enrolled starting $1^{\text {st }}$ of June 2015 until 30 $30^{\text {th }}$ of March 2018, of which 116 were enrolled in the control group, and 120 in the simulation group. Baseline characteristics are presented in Table 1.

Table $1 \quad$ Baseline characteristics.

Characteristic

Control $(n=116)$

Simulation $(n=120)$

\begin{tabular}{|c|c|c|}
\hline Age (years) & $67.5(56-75)$ & $68.0(56-75)$ \\
\hline Male & $79(68)$ & $71(60)$ \\
\hline \multicolumn{3}{|l|}{ Comorbidity } \\
\hline Diabetes & $49(42)$ & $46(39)$ \\
\hline Hypertension & $97(83)$ & $105(88)$ \\
\hline Peripheral arterial disease & $18(16)$ & $24(20)$ \\
\hline Cardiovascular disease & $43(37)$ & $51(43)$ \\
\hline Cerebrovascular disease & $16(14)$ & $24(20)$ \\
\hline \multicolumn{3}{|l|}{ Medication } \\
\hline Coagulation & $51|17| 0$ & $49|22| 2$ \\
\hline ACE-inhibitor & $41(35)$ & $31(26)$ \\
\hline Statin & $70(60)$ & $68(57)$ \\
\hline \multicolumn{3}{|l|}{ AVF configuration ${ }^{1}$} \\
\hline RCAVF & $51(48)$ & $51(46)$ \\
\hline BCAVF & $41(38)$ & $37(33)$ \\
\hline BBAVF & $11(10)$ & $16(14)$ \\
\hline Other* & $4(4)$ & $10(9)$ \\
\hline
\end{tabular}

Age is reported as median (IQR), all other variables are counts (percentage). Coagulation are antiplatelet drugs, vitamin $\mathrm{K}$ antagonists (VKA), and non-VKA oral anticoagulants respectively. ${ }^{1}$ The distribution of AVF configurations is tested for significance: Chi-square 3.484, $p=.323 .{ }^{*}$ These include brachiocubital AVFs, configuration with high arterial bifurcation, and arteriovenous grafts. RC-: radiocephalic; BC-: brachiobasilic; BBAVF: brachiobasilic arteriovenous fistula.

For AVF maturation 205 (87\%) patients were available for analysis. The reasons for unavailability are summarized in Table 2, of which death was the main reason. Thrombosed AVFs are considered as failed to mature. Direct postoperative flow measurement was not a standard follow-up visit in all hospitals; therefore model 
performance could be assessed in 132 patients. For this endpoint the patients randomized for the control group were also simulated. Recommendation compliance was available for 114 simulation patients.

Table 2 Availability for primary endpoint assessment.

\begin{tabular}{r|cc|}
\multicolumn{2}{c}{ Control (n=116) } & Simulation (n=120) \\
\hline $\begin{array}{r}\text { Available } \\
\text { Not available }\end{array}$ & $100(86)$ & $105(88)$ \\
Withdrew consent & $16(14)$ & $14(12)$ \\
Transplanted before AVF & 3 & 1 \\
Death & 3 & 0 \\
Missed FU visit & 5 & 1 \\
No AVF & 1 & 0 \\
AVG placement & 2 & 4 \\
\cline { 2 - 2 }
\end{tabular}

The two patients that missed the FU visit were due to logistical issues in a hospital that changed their electronic health records and planning systems. Two patients in the control group refrained from AVF placement after inclusion. Grafts were placed for an urgent dialysis indication and/or inadequate vessels at surgical exploration. AVF: arteriovenous fistula; FU: follow-up; AVG: arteriovenous graft.

\section{Primary outcome - arteriovenous fistula maturation}

FTM rates were similar in the control and simulation group, with 29 and 32\% respectively $(p=0.600)$. In the control group 29 patients failed to mature their fistulae. This FTM was due to early thrombosis in 6, and 23 AVFs did not meet the DUS criteria for maturation. For the simulation group $(n=34)$ these numbers were 7 and 27 respectively. Subanalysis of FTM rates for RC-, BC- and BBAVFs (control and simulation group combined) were statistically different with 39,23 , and $15 \%$ respectively $(p=0.014)$. The number of the various other configurations were too few and too heterogeneous to be included in this subanalysis. No operative complications were documented.

AVF configurations did not differ between the two groups $(p=0.323)$. The RCAVF is with 48 respectively $46 \%$ the predominant configuration in both patient groups (Table 1).

\section{Secondary outcome - maturation predictors}

Univariate analysis showed that exclusively immediate postoperative AVF flow prediction $(881 \pm 447$ vs $669 \pm 321 \mathrm{~mL} / \mathrm{min})$ was significantly associated with AVF maturation. Table 3 shows the results for univariate analysis for the potential 
dichotomous predictors. Age, as a continuous variable, did not differ between the matured and FTM groups (67.5 [56-75] vs 68.0 [56-75] years respectively). In the logistic model the odds ratio of the flow prediction for maturation was 1.001 (1.001-1.002; Table 4). Figure 2 represents the corresponding ROC curve, with an AUC of 0.67 $(p<0.001)$.

\section{Table 3 Univariate analysis of arteriovenous fistula maturation predictors.}

\begin{tabular}{|c|c|c|}
\hline & Odds Ratio & $95 \% \mathrm{Cl}$ \\
\hline Male & 1.126 & $0.606-2.092$ \\
\hline Diabetes & 1.214 & $0.654-2.251$ \\
\hline PAD & 1.048 & $0.450-2.441$ \\
\hline Cardiovascular disease & 1.293 & $0.691-2.418$ \\
\hline Cerebrovascular disease & 0.757 & $0.356-1.610$ \\
\hline Hypercholesterolemia & 0.681 & $0.413-1.123$ \\
\hline Race & 2.023 & $0.951-4.303$ \\
\hline Regional anesthesia & 0.809 & $0.422-1.552$ \\
\hline On dialysis & 1.245 & $0.658-2.355$ \\
\hline
\end{tabular}

The predictors in this table were based on literature, and exclusively white race (i.e. white vs. other) was statistically significant. Age and flow prediction were assessed on a continuous scale and therefore not reported in this table. PAD: peripheral arterial disease; $95 \% \mathrm{Cl}$ : $95 \%$ confidence interval for the odds ratio.

Table 4 Regression model for arteriovenous fistula maturation.

\begin{tabular}{|c|c|c|c|c|c|}
\hline & B & S.E. & Wald & OR & Sig. \\
\hline Flow prediction & .001 & .000 & 10.159 & $1.001(1.001-1.002)$ & .001 \\
\hline Constant & -.350 & .360 & 0.946 & 0.704 & \\
\hline
\end{tabular}

Flow prediction is entered on a continuous scale $(\mathrm{mL} / \mathrm{min})$, which means that for each $\mathrm{mL} / \mathrm{min}$ increase of the flow prediction, the odds on maturation increases 1.001. The accompanying -2Log likelihood is 234.093 , and the Nagelkerke $R^{2}$.083. B: coefficient; S.E: standard error; OR: odds ratio; Sig: significance. 


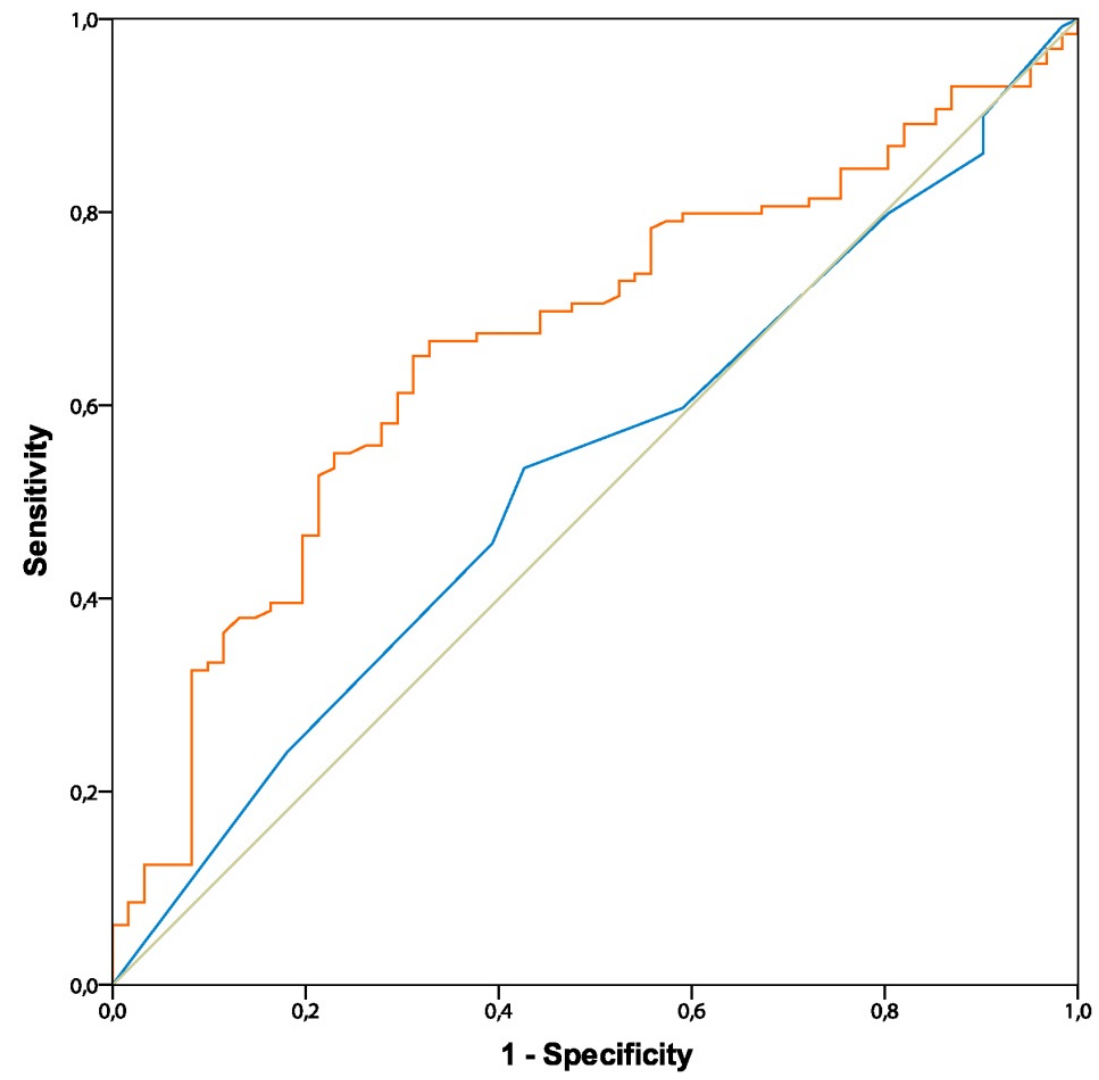

Figure 2 Receiver Operator Characteristic (ROC) Curve. The area under the curve (AUC) for the flow predictions is 0.67 ( $95 \% \mathrm{Cl} 0.59-0.75 ; \mathrm{p}<0.001$; orange line) whereas it is $0.53(0.44-0.62 ; \mathrm{p}=0.517$; blue line) for the REDUCE-FTM score. Reference line (beige) denotes an equal or random chance, which corresponds with an AUC of 0.5 .

\section{Model performance}

Average immediate postoperative measured flow was $920 \pm 464$ versus $480 \pm 270 \mathrm{~mL} / \mathrm{min}$ for matured and FTM fistulae respectively $(p<0.001)$. Although immediate postoperative flow differed significantly between mature and FTM fistulae, there is large overlap (Figure 3). The respective average predicted postoperative flow was $881 \pm 447$ and $669 \pm 321 \mathrm{~mL} / \mathrm{min}(\mathrm{p}<0.01)$. Subanalysis for clinical outcome showed that there was no difference between measured and predicted postoperative flow for the maturation group (i.e. $925 \pm 473$ vs. $819 \pm 399, p=0.067$ ), whereas for the FTM group there was (i.e. $480 \pm 270$ vs. $653 \pm 339 \mathrm{~mL} / \mathrm{min} ; \mathrm{p}=0.004$ ). The positive predictive value for the model threshold of $400 \mathrm{~mL} / \mathrm{min}$ is $83 \%$. The other diagnostic values (sensitivity, specificity, and negative predictive value) are summarized in Table 5 . 


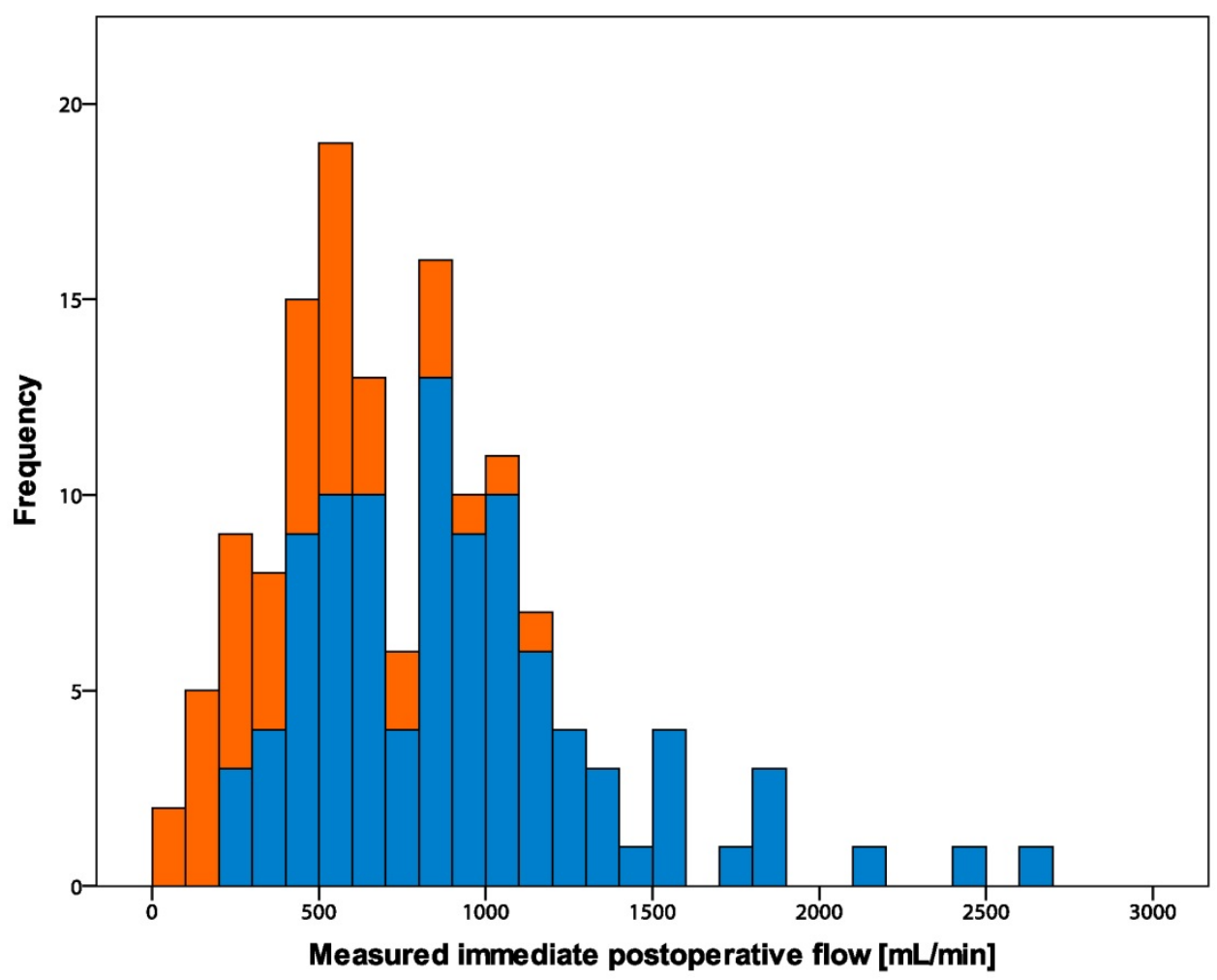

Figure 3 Histogram of immediate postoperative flow for FTM and mature fistulae. Although there is a statistically significant difference in mean postoperative flow, the two groups show large overlap limiting discriminating both groups. Orange denotes fistulae that failed to mature, whereas blue denotes mature fistulae.

Table 5 Model diagnostic values.

\begin{tabular}{|c|c|c|c|}
\hline \multirow[b]{2}{*}{ Flow prediction [mL/min] } & \multicolumn{2}{|c|}{ Flow measurement [mL/min] } & \multirow[b]{2}{*}{ Total } \\
\hline & $\geq 400$ & $<400$ & \\
\hline$\geq 400$ & 96 & 20 & 116 \\
\hline$<400$ & 13 & 3 & 16 \\
\hline Total & 109 & 23 & 132 \\
\hline
\end{tabular}

Of 132 patients direct postoperative flow predictions and measurements were available, and categorized for surpassing the recommendation cut-off values. The following diagnostic values are calculated based on this table: sensitivity $88 \%(96 / 109)$, specificity $13 \%$ (3/23), positive predictive value $83 \%(96 / 116)$, and negative predictive value $19 \%(3 / 16)$. 


\section{Recommendation compliance}

In $12 \%$ of the cases the model recommendation changed the initial surgical plan, in $57 \%$ the recommendation and surgical plan were in agreement, in $25 \%$ the recommendation was not acted upon by the physician, and in $6 \%$ there was no recommendation. Reasons for not following the recommendation $(n=28)$ were: peroperative changes of the surgical plan $(n=7)$, inaccurate representation of the anatomy for simulation (e.g. thrombosed cephalic vein; $n=6$ ), preoperative DUS not in agreement with findings at physical examination (e.g. visible cephalic vein, but small diameters at DUS; $n=4$ ), urgent dialysis indication ( $n=4$, of which two received an early cannulation graft), no confidence in the flow predictions (i.e. surprisingly low flow predictions in adequate vessel diameters; $n=3$ ), and marginally different flow predictions between two configurations (e.g. BC- and BBAVF almost similar predictions; $n=2$ ). Only one of seven cases in which no recommendation was given was due to nonconverging simulations, whereas in the other six all three configurations were under or above the defined cut-off values.

\section{DISCUSSION}

To improve AVF maturation rates a computational model that patient-specifically predicts immediate postoperative flows was implemented in regular health care. These flow predictions were found to be significant predictors for AVF maturation. Despite this finding, maturation rates were not improved when the vascular surgeons had the flow predictions at their disposal during surgical planning. In both groups FTM rates were approximately $30 \%$. This proportion is in agreement with the range reported in other studies (i.e. 20-40\%). 3, 4, 17, 19

Patient-specific flow predictions are an innovative approach to improve maturation rates. Flow is an important determinant for AVF maturation, and providing flow predictions during surgical planning might improve maturation rates. The computational model has been found to reliably predict immediate postoperative blood volume flows, and could therefore be usable for clinical decision-support. ${ }^{12,13,20}$

Previously, researchers have failed to improve maturation rates by altering the anastomotic technique, ${ }^{21,22}$ introduction of anastomotic devices, systemic drug therapy (e.g. anticoagulants, statins, ACE inhibitors ${ }^{23}$ ), or risk prediction models. ${ }^{8,24,25}$ The latter do not compare with the model employed here, since risk classification scores are based on statistical models, and therefore are neither truly patient-specific, nor are they readily generalizable. 
The REDUCE-FTM ${ }^{8}$ and DISTAL ${ }^{25}$ scores aim to predict maturation likelihood for all AVF configurations respectively exclusively snuffbox AVFs, and the $C A V e A_{2} T_{2}$ score predicts the likelihood of secondary patency of RCAVFs. ${ }^{24}$ These three models could predict the outcome of interest for their specific populations, but failed external validation. The DISTAL and CAVeA $\mathrm{T}_{2}$ scores are not externally validated, whereas the REDUCE-FTM lost its predictive power when applied to other than the Canadian population. The AUC of the REDUCE-FTM score in our study was only $0.53(0.44-0.62)$, which is comparable to other studies that reported the C-statistic (i.e. a measure of goodness of fit comparable to the AUC of a ROC curve) of the score in their population. In Ireland the C-statistic was 0.53, and in the United States 0.52. ${ }^{4,10}$

The AUC of the regression model for patient-specific flow predictions was 0.67 ([0.58-0.75]; $\mathrm{p}<0.001)$. Although this indicates a poor test, it is an encouraging result for several reasons. Firstly, this is the first result of the model when actually applied in clinical decision-making, and then performs better than chance (i.e. AUC 0.5). Secondly, the model can become fair or better (i.e. AUC $\geq 0.70$ ) when the model accuracy and/or model input is improved according to the insights from the present study, especially when the overestimation of postoperative flow in failing AVFs is addressed. However, the AUC of flow predictions can never become 1.00, since the AUC of postoperative flow measurements is approximately 0.80 . And, thirdly, it outperforms the REDUCE-FTM score, which is more or less the gold standard of FTM risk scores.

The results of the flow predictions will be more robust in comparison to risk prediction models. The latter depend on demographical factors that vary between populations, whereas the flow predictions depend on physics. The computational model is therefore not geographically or demographically confined and should perform equal for instance in The Netherlands, Canada, Ireland and the United States. The AUC or C-statistic might decrease - as usually happens on external validation of prediction models - but presumably not as much as the REDUCE-FTM score (i.e. from 0.74 to \pm 0.52 ). $4,8,10$

Despite the predictive power of the flow predictions, maturation rates did not improve. On the one hand, this is due to inaccuracy in predictions, while on the other hand, there is more to maturation than just postoperative flow (e.g. venous diameter increase, adequate response to hemodynamical changes). ${ }^{26} \mathrm{Immediate}$ postoperative flows, albeit significantly different between matured and FTM fistulae, show large overlap, and even the FTM fistulae had an immediate postoperative flow of $480 \pm 270 \mathrm{~mL} / \mathrm{min}$. Farrington et al. recently found that postoperative blood flow with an OR 1.33 (1.22-1.45) per 100 $\mathrm{mL} / \mathrm{min}$ increase, was predictive for AVF maturation. ${ }^{27}$

When we analyzed our data per $100 \mathrm{~mL} / \mathrm{min}$ increase similar results were found. For immediate postoperative flow measurements the OR is 1.49 (1.27-1.75). The OR for the immediate postoperative flow predictions is $1.15(1.06-1.26)$ per $100 \mathrm{~mL} / \mathrm{min}$. Although 
this is lower than for the measured flow, it has the obvious benefit that this predictor is available without actually creating the AVF.

Measured and predicted flow significantly differed for FTM fistulae, which is indicative that FTM (at least in part) is an inadequate response to the altered hemodynamics. Malovrh identified a positive relation between a higher (i.e. $\geq 0.7$ ) resistance index at reactive hyperemia and AVF failure. ${ }^{28}$ Conversely, in the Hemodialysis Fistula Maturation a positive relation was found between flow- and nitroglycerine mediated dilatation (FMD respectively NMD) and 6 week AVF flow and diameter development. ${ }^{29}$

Functional abnormalities most likely explain FTM better than cellular/histological abnormalities. Preexisting (i.e. before AVF creation) microcalcification, medial fibrosis, intimal hyperplasia, accumulation of extracellular matrix, have been extensively studied in relation to AVF maturation. Although almost all aforementioned abnormalities are consistently found to be present in the majority of hemodialysis patients, they do not explain FTM. ${ }^{30-32}$

The large agreement between the initial surgical plan and recommendation also contributed to a lack of an effect on maturation rates. In $57 \%$ of the cases these were in agreement, reducing the possibility to improve maturation rates. In $25 \%$ of the patients the physicians did not follow the recommendation, and in the majority of these cases this was not due to model malperformance, but to intra-operative strategy change, urgent dialysis and subsequently need for early access, and differences between findings at DUS and physical examination.

Conversely, the inaccurate representation of the anatomy is related to malperformance. The model was designed to interpolate missing data (i.e. a missing discrete measurement). When these data were missing due to missing anatomy (e.g. a thrombosed upper arm cephalic vein) these data would also be interpolated, and used for simulation. Consequently, the model would also predict an immediate postoperative BCAVF flow although this configuration is not anatomically available. Future model improvement should therefore be optimized to adequately deal with sparse anatomical data due to missing data or missing anatomy.

In six out of seven cases in which no configuration was recommended this was due to the fact that all configurations were below and/or above predefined thresholds. Predicted immediate postoperative flow should be $\geq 400 \mathrm{~mL} / \mathrm{min}$ for maturation, but not exceed $1500 \mathrm{~mL} / \mathrm{min}$ due to a presumed high risk on high flow complications. These thresholds were based on literature, ${ }^{16,33}$ and should be improved in future studies based on the results of the present study.

The future direction of the flow prediction model might be using it in a hybrid model, for example as a covariate in risk prediction scores, or as a node in artificial neural networks (i.e. artificial intelligence). Implementing flow predictions in risk prediction models has 
the benefit of incorporating a continuous variable that is based on physics, and therefore does not depend on the sample or population it is applied to. Which additional covariates should be implemented in such models has to be determined, but becomes increasingly confusing.

The three aforementioned prediction models incorporate in total 15 variables, but share only two: ischemic heart disease (in REDUCE-FTM and DISTAL) and peripheral arterial disease (REDUCE-FTM and $C A V e A_{2} T_{2}$ ). Interestingly, neither ischemic heart disease nor peripheral arterial disease was confirmed by the external validation studies of the REDUCE-FTM score. Conversely, the external validation studies in turn identified other FTM risk factors such as female sex or race (i.e. non-Caucasian). In the present study none of the reported risk factors was found to be of influence for AVF maturation failure.

Using artificial intelligence or more direct measures of vascular or overall health are two possibilities to finally identify unequivocal risk factors. Examples of more direct measures are using $\mathrm{HbA} 1 \mathrm{c}$ or pulse wave velocity measurement instead of diabetes respectively hypertension as is now the case. Many studies - our study included - dichotomize complex diseases with varying severity and/or duration such as diabetes. Maybe $\mathrm{HbA} 1 \mathrm{c}$ is then more appropriate for FTM risk factor identification, as it is a measure for chronic glycemic variability and does not dichotomize the diabetes to present or absent. The same goes for PWV measurement instead of scoring hypertension as present or absent, which is on a continuous scale and depends on arterial stiffness.

Artificial intelligence on the other hand can be used to find hidden insights, and therewith find new FTM risk factors. All factors that are currently studied are based on theory or results of other studies. Certainly, there are still unknown factors but identifying these is very laborious if not impossible by conventional ways. Artificial intelligence, however, can find these insights without explicit programming where to look, and integrate and interpret data in ways traditional statistical models cannot. Although promising results are reported in other medical fields and specifically in cardiovascular medicine, it has yet to be applied to arteriovenous fistula research. ${ }^{34,35}$

\section{Limitations}

The present study has two limitations. Firstly, since in approximately $60 \%$ the recommendation was in agreement with the initial surgical plan any effect of the model can be obscured. Secondly, the primary endpoint is not a clinical endpoint - it does not give any information on the functionality of the AVF. However, it is an objective measure, and when patients reach the criteria of $\geq 500 \mathrm{~mL} / \mathrm{min}$ and $4 \mathrm{~mm}$ used in this trial, there is a $95 \%$ likelihood that the AVF will become functional. ${ }^{2}$ Furthermore, to assess functionality, included patients should depend on hemodialysis, and it is still 
difficult to predict which and when a predialysis patient will progress to dialysis dependency. ${ }^{36}$

\section{Generalizability}

The results of this trial are readily generalizable. Firstly, practically all (pre)hemodialysis patients in need of an AVF were eligible for inclusion. Secondly, it was designed in such a fashion that it can be easily implemented in daily practice. Usually Dutch hospitals have well-equipped vascular laboratories with trained technicians who routinely perform preoperative DUS examinations for AVF surgery. For the purpose of the study DUS protocols were standardized to comply with the model's demands. The multicenter design of the present study itself is also a major strength, as it shows that the model can be implemented in other clinics. Thirdly, hospital selection ensured a representative sample of the Dutch dialysis population that appears to be comparable with other European studies. ${ }^{4} 19$ In comparison to North American studies, there is however a considerable lower percentage of blacks included. 8,10

\section{Interpretation}

In conclusion, this innovative approach of patient-specifically simulating immediate postoperative flows is a strong predictor for AVF maturation, with the added benefit of having postoperative flows without actual surgery. Since AVF maturation importantly but not exclusively depends on flow, the predictive power of separate model use is insufficient to support clinical decision-making. Incorporating the flow prediction in hybrid modeling approaches - in which other relevant but non-physical parameters or factors can be incorporated - might be beneficial, especially when the accuracy of the flow predictions in FTM fistulae is improved.

\section{OTHER INFORMATION}

\section{Registration}

This trial is registered at ClinicalTrials.gov (NCT02453412), and ToetsingOnline.nl (51610.068.14). 
140 | CHAPTER 6

\section{Protocol}

Study protocol is published previously by Zonnebeld et al. ${ }^{14}$

\section{Funding}

This trial is funded by the Dutch Kidney Foundation (NT12.01). 


\section{REFERENCES}

1. Ravani P, Palmer SC, Oliver MJ, et al. Associations between hemodialysis access type and clinical outcomes: a systematic review. Journal of the American Society of Nephrology. 2013;24(3):465-73.

2. Robbin ML, Chamberlain NE, Lockhart ME, et al. Hemodialysis arteriovenous fistula maturity: US evaluation. Radiology. 2002;225(1):59-64.

3. Huijbregts $\mathrm{HJ}$, Bots $\mathrm{ML}$, Wittens $\mathrm{CH}$, et al. Hemodialysis arteriovenous fistula patency revisited: results of a prospective, multicenter initiative. Clinical journal of the American Society of Nephrology. 2008;3(3):714-9.

4. Masengu A, Maxwell AP, Hanko JB. Investigating clinical predictors of arteriovenous fistula functional patency in a European cohort. Clinical kidney journal. 2016;9(1):142-7.

5. System USRD. USRDS annual data report: An overview of the epidemiology of kidney disease in the United States. 2017; Volume Two: End-Stage Renal Disease in the United States.

6. Kramer A, Pippias M, Noordzij M, et al. The European Renal Association - European Dialysis and Transplant Association (ERA-EDTA) Registry Annual Report 2015: a summary. Clinical kidney journal. 2018;11(1):108-22.

7. Georgiadis GS, Charalampidis DG, Argyriou C, et al. The Necessity for Routine Preoperative Ultrasound Mapping Before Arteriovenous Fistula Creation: A Metaanalysis. European journal of vascular and endovascular surgery. 2015;49(5):600-5.

8. Lok CE, Allon M, Moist $\mathrm{L}$, et al. Risk equation determining unsuccessful cannulation events and failure to maturation in arteriovenous fistulas (REDUCE FTM I). Journal of the American Society of Nephrology. 2006;17(11):3204-12.

9. Al Shakarchi J, McGrogan D, Van der Veer S, et al. Predictive models for arteriovenous fistula maturation. The journal of vascular access. 2016;17(3):229-32.

10. Lilly MP, Lynch JR, Wish JB, et al. Prevalence of arteriovenous fistulas in incident hemodialysis patients: correlation with patient factors that may be associated with maturation failure. American journal of kidney diseases. 2012;59(4):541-9.

11. Huberts W, Bode AS, Kroon W, et al. A pulse wave propagation model to support decision-making in vascular access planning in the clinic. Medical engineering \& physics. 2012;34(2):233-48.

12. Bode AS, Huberts W, Bosboom EM, et al. Patient-specific computational modeling of upper extremity arteriovenous fistula creation: its feasibility to support clinical decision-making. PloS one. 2012;7(4):e34491.

13. Caroli A, Manini S, Antiga $L$, et al. Validation of a patient-specific hemodynamic computational model for surgical planning of vascular access in hemodialysis patients. Kidney international. 2013;84(6):1237-45.

14. Zonnebeld N, Huberts W, van Loon MM, et al. Preoperative computer simulation for planning of vascular access surgery in hemodialysis patients. The journal of vascular access. 2017;18(Suppl. 1):118-24.

15. Boutron I, Altman DG, Moher D, et al. CONSORT Statement for Randomized Trials of Nonpharmacologic Treatments: A 2017 Update and a CONSORT Extension for Nonpharmacologic Trial Abstracts. Ann Intern Med. 2017;167(1):40-7. 
16. Shemesh D, Goldin I, Berelowitz D, et al. Blood flow volume changes in the maturing arteriovenous access for hemodialysis. Ultrasound in medicine \& biology. 2007;33(5):727-33.

17. Al-Jaishi AA, Oliver MJ, Thomas SM, et al. Patency rates of the arteriovenous fistula for hemodialysis: a systematic review and meta-analysis. American journal of kidney diseases. 2014;63(3):464-78.

18. Carter JV, Pan J, Rai SN, et al. ROC-ing along: Evaluation and interpretation of receiver operating characteristic curves. Surgery.159(6):1638-45.

19. Wilmink T, Hollingworth L, Powers S, et al. Natural History of Common Autologous Arteriovenous Fistulae: Consequences for Planning of Dialysis Access. European Journal of Vascular and Endovascular Surgery. 2016;51(1):134-40.

20. Bozzetto M, Rota S, Vigo V, et al. Clinical use of computational modeling for surgical planning of arteriovenous fistula for hemodialysis. BMC Med Inform Decis Mak. 2017;17(1):26.

21. Bharat A, Jaenicke $M$, Shenoy S. A novel technique of vascular anastomosis to prevent juxta-anastomotic stenosis following arteriovenous fistula creation. Journal of vascular surgery. 2012;55(1):274-80.

22. Sadaghianloo N, Declemy S, Jean-Baptiste E, et al. Radial artery deviation and reimplantation inhibits venous juxta-anastomotic stenosis and increases primary patency of radial-cephalic fistulas for hemodialysis. Journal of vascular surgery. 2016;64(3):698-706 e1.

23. Viecelli AK, Mori TA, Roy-Chaudhury $P$, et al. The pathogenesis of hemodialysis vascular access failure and systemic therapies for its prevention: Optimism unfulfilled. Seminars in dialysis. 2018;31(3):244-57.

24. Bosanquet DC, Rubasingham J, Imam M, et al. Predicting outcomes in native AV forearm radio-cephalic fistulae; the CAVeA2T2 scoring system. The journal of vascular access. 2015;16(1):19-25.

25. Twine $C P$, Haidermota $M$, Woolgar JD, et al. A scoring system (DISTAL) for predicting failure of snuffbox arteriovenous fistulas. European journal of vascular and endovascular surgery : the official journal of the European Society for Vascular Surgery. 2012;44(1):88-91.

26. Hentschel DM. Determinants of Arteriovenous Fistula Maturation. Clinical journal of the American Society of Nephrology. 2018.

27. Farrington $C A$, Robbin $M L$, Lee $T$, et al. Postoperative Ultrasound, Unassisted Maturation, and Subsequent Primary Patency of Arteriovenous Fistulas. Clinical journal of the American Society of Nephrology. 2018.

28. Malovrh M. Native arteriovenous fistula: preoperative evaluation. American journal of kidney diseases. 2002;39(6):1218-25.

29. Allon M, Greene T, Dember LM, et al. Association between Preoperative Vascular Function and Postoperative Arteriovenous Fistula Development. Journal of the American Society of Nephrology. 2016;27(12):3788-95.

30. Alpers CE, Imrey PB, Hudkins KL, et al. Histopathology of Veins Obtained at Hemodialysis Arteriovenous Fistula Creation Surgery. Journal of the American Society of Nephrology. 2017;28(10):3076-88. 
31. Allon M, Robbin ML, Umphrey HR, et al. Preoperative arterial microcalcification and clinical outcomes of arteriovenous fistulas for hemodialysis. American journal of kidney diseases. 2015;66(1):84-90.

32. Allon $M$, Litovsky $S$, Young $C J$, et al. Medial fibrosis, vascular calcification, intimal hyperplasia, and arteriovenous fistula maturation. American journal of kidney diseases. 2011;58(3):437-43.

33. Tordoir JH, Dammers $\mathrm{R}$, van der Sande FM. Upper extremity ischemia and hemodialysis vascular access. European journal of vascular and endovascular surgery. 2004;27(1):1-5.

34. Shameer K, Johnson KW, Glicksberg BS, et al. Machine learning in cardiovascular medicine: are we there yet? Heart. 2018;104(14):1156-64.

35. Deo RC. Machine Learning in Medicine. Circulation. 2015;132(20):1920-30.

36. Al-Balas A, Lee T, Young CJ, et al. Predictors of Initiation for Predialysis Arteriovenous Fistula. Clinical journal of the American Society of Nephrology. 2016;11(10):1802-8.

37. Zonnebeld N, Maas TMG, Huberts W, et al. Pre-operative Duplex Ultrasonography in Arteriovenous Fistula Creation: Intra- and Inter-observer Agreement. European journal of vascular and endovascular surgery. 2017;54(5):613-9. 


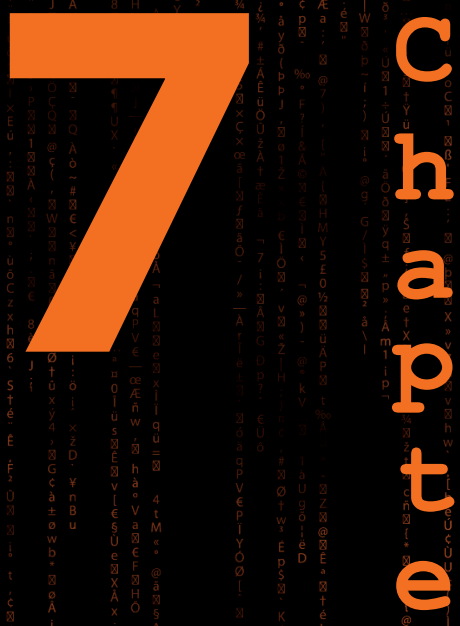

I

\section{Surgical and Endovascular Interyention for Dialysis Access Maturation Failure During and After Arteriovenous Fistula Surgery: Review of the Evidence}

Jan. H:M. Tordoii,, ${ }^{1}$ Niek Zonnebeld, ${ }^{1}$ Magda M. van Loon, ${ }^{1}$ Măurizio Gallieni, ${ }^{2}$ Markus Hollenbeck ${ }^{3}$

Eur J Vàsc Endovasc Surg (2018) 55, 240-248

1. Department of Vascular Surgery, Maastricht University Medicăl Center, Maastricht, The Netherlands

2. Nểphrology and Dialysis Unit, Ospedale San Carlo Borrơmeo, University of Milanô, Millan, Italy 3. Department of Nephrology and Rheumatology, Knappschaftskrankenhaus Bottrop, Bottrop, Germany 


\section{ABSTRACT}

Maturation failure is the major obstacle to establishing functional arteriovenous fistulae (AVF) for haemodialysis treatment. Various endovascular and surgical techniques have been advocated to enhance fistula maturation and to increase the number of functional AVFs. This narrative review considers the available evidence of interventional techniques for treatment of AVF non-maturation.

\section{Results}

Intra-operative vein dilation and anastomosis modification results in a clinical maturation rate of $74-92 \%$ and a 6 month cumulative AVF patency of $79-93 \%$. Percutaneous transluminal angioplasty (PTA) with or without accessory vein obliteration is successful in $43-97 \%$ of patients. The long-term primary patency of PTA is rather low and multiple re-interventions are needed to achieve an acceptable cumulative fistula patency. The results of surgical revision exceed the results of endovascular intervention, with a mean primary one year patency of $73 \%$ (range $68-78 \%$ ) compared with $49 \%$ (range 28-72\%), respectively. The role of accessory vein obliteration remains unclear.

\section{Conclusion}

Intervention for autologous arteriovenous fistula non-maturation is worthwhile and results in an increased number of functional fistulae. The outcome of surgical revision is better than endovascular and might be preferable in certain patient populations. 


\section{INTRODUCTION}

Autologous arteriovenous fistulae (AVF) have been recognized as the primary and best hemodialysis vascular access option in patients with end stage renal disease (ESRD) who require renal replacement therapy. ${ }^{1,2}$ The benefits of autologous AVFs in comparison with prosthetic arteriovenous grafts (AVG) and central vein catheters (CVC) are obvious in terms of low thrombosis and infection rates, fewer hospital admissions for access revision, and lower healthcare related costs. ${ }^{3}$ In addition, patients with an AVF have substantially lower mortality rates and increased life expectancy. ${ }^{4}$

The creation of a functional AVF is hampered by the relatively high early thrombosis and failure to mature (FTM) rate. Contemporary studies have reported $24 \%$ and $60 \%$ FTM rates in European and American hemodialysis patients, respectively. ${ }^{5-8}$ In AVFs that fail to mature, the juxta-anastomotic vein (JAV) does not increase in luminal size because of the local hemodynamic fluid conditions. On the other hand, vein size may be sufficient, but blood flow is not enhanced because of lack of adequate arterial inflow and remodeling, for instance in patients with calcified vessels. ${ }^{9,} 10$ These conditions trigger AVF non-maturation and may be different between patients depending on patient demographics and comorbidities.

A meta-analysis reported a growing percentage of patients with non-functioning AVFs because of maturation failure in recent decades. ${ }^{11}$ This observation might be largely a result of a change in patient demographics, with advanced age, diabetes mellitus, and nephrosclerosis as leading causes of ESRD. In these patients, calcified and noncompliant arteries and sclerotic veins might be responsible for failed vessel adaptation, resulting in low arteriovenous (AV) blood flow and impaired vessel diameter increase. ${ }^{12}$

Intervention for fistula maturation failure might be worthwhile and may result in an acceptable vascular access outcome. A systematic review of endovascular and surgical intervention has shown a fistula salvage rate of $74-97 \%$. The primary and secondary one year patency rates varied from $28 \%$ to $68 \%$ and $72 \%$ to $95 \%$, respectively. ${ }^{13}$ This review looked exclusively at post-surgical interventional methods and not at the outcome of the various surgical and endovascular methods during fistula creation, for the enhancement of access maturation.

The present review is an analysis of current techniques to review the evidence for operative and post-operative endovascular and surgical anastomotic modifications and interventions for the purpose of improved autologous arteriovenous fistula maturation. 


\section{LITERATURE SEARCH}

A search syntax consisting of synonyms of the following combination of terms was constructed to conduct a computer assisted search of the MEDLINE, EMBASE, and Cochrane libraries, from the inception of these databases until June 30, 2017: "arteriovenous fistula" and "haemodialysis patients" and "maturation" or "surgery" or "endovascular methods" or "risk factors" and "AVF outcome" and "balloon assisted maturation," "clipped anastomosis," "anastomotic device." A total of 405 publications were found, and after selection 55 publications remained eligible for review.

\section{RESULTS}

\section{Operative surgical and endovascular methods to improve arteriovenous fistula maturation}

\section{Adaptation of surgical technique}

One randomized study on the use of nitinol clips for AV anastomosis compared with sutured anastomosis, showed a trend to better primary and assisted primary patency of clipped fistulae, although the differences were not statistically significant. The 6 month primary patency rate was $61 \%$ with sutures and $69 \%$ with clips ( $p=$ not significant). There were no significant differences in the number of revisions or other morbidity. ${ }^{14}$ Three non-randomized studies showed different outcomes of the use of titanium and nitinol clips for AV anastomosis. Clipped radiocephalic (RC) and brachiocephalic (BC) AVF had an improved maturation rate at 6 weeks $(86 \%$ vs. $69 \%, p<.05)$ and primary patency rates at 12,24 , and 36 months were $91 \%, 84 \%$, and $75 \%$ for the clipped AVFs and $83 \%$, $74 \%$, and $61 \%$ for the sutured AVFs ( $p<.05)$. However, there was no difference in the secondary patency at 12,24 , or 36 months $(91 \%, 80 \%$, and $75 \%$ vs. $83 \%, 77 \%$, and $69 \%$, respectively). The second study of 334 fistulae ( $29 \%$ suture and $71 \%$ clips) in 326 patients showed no difference in maturation rate (suture vs. clips $79 \%$ and $72 \%$ ), median time to maturation (suture vs. clips 62 and 71 days), one year primary patency rate (suture vs. clips $37 \%$ and $40 \%$ ), one year assisted primary patency rate (suture vs. clips $82 \%$ and $76 \%$ ), or overall failure rates (suture vs. clips $62 \%$ and $58 \%$ ). Median time to initial failure or reintervention was not significantly different in the clip group (suture vs. clips 615 and 812 days). ${ }^{15,16}$ The third study described the use of U-CLIP devices in the surgical creation of AVFs in 68 patients. Overall, 61 of the 65 fistulae created, matured successfully within 
8-9 weeks; $93 \%$ of the RCAVFs, $69 \%$ of the BCAVFs, and $100 \%$ of the basilica vein transpositions, respectively. ${ }^{17} \mathrm{~A}$ randomized controlled trial comparing interrupted $(n=42)$ with continuous $(n=36)$ suturing techniques for RCAVF creation, showed a better primary patency $(71 \%$ vs. $47 \% ; p=.01)$, but similar functional patencies at 6 weeks $(52 \%$ versus $36 \%)^{18}$

The SLOT (Straight Line Onlay Technique) and RADAR (Radial Artery Deviation And Reimplantation) AV anastomosis techniques both have improved fistula outcomes with less juxta-anastomotic stenosis (JAS) development. For the SLOT technique, the cephalic vein that lies in the subcutaneous plane is dissected and the cut end is over sewn. It is moved medially over the artery that lies in a deeper plane (underneath the deep fascia). A fistula is created between the posterior aspect of the vein (underside of the vein) and the anterior aspect of the artery. The outflow vein is dissected further in the subcutaneous tissue to obtain straight lie. Thus, the vein "piggybacks" on the artery at the site of anastomosis, and the arterial blood flows into a straight cylindrical lumen. A non-randomized study of three different anastomotic techniques (end to side [ETS]; side to side [STS] vein to artery and SLOT) in RCAVFs created in 125 patients showed a significant decrease in JAS development in the SLOT patients $(p=.04)$. SLOT patients also had decreased overall fistula failure (ETS 40.3\%, STS 33.3\%, SLOT 16.7\%; $p=.01$ ). ${ }^{19}$ Recently, long-term results of the SLOT technique were published with one year primary and secondary patencies of $42.8 \%$ and $81.8 \%$, respectively. ${ }^{20}$ Instead of using a traditional end vein to side artery anastomosis, RADAR uses an end artery to side vein anastomosis, coupled with conventional "no-touch" techniques and advocate avoidance of any arterial and venous dissection or manipulation. The RADAR fistulae have increased rates of maturation compared with control fistulae, $75 \%$ versus $45 \%$ at 6 weeks $(p<.001), 92 \%$ versus $71 \%$ at 3 months, $(p<.003)$ and decreased incidence of JAS ( $2 \%$ vs. $41 \% ; p<.001)$. At 6 months, RADAR fistulae had increased primary patency $(93 \%$ vs. $53 \% ; \mathrm{p}<.0001)$ and secondary patency $(100$ vs. $89 \% ; \mathrm{p}=.0003)$ and decreased incidence of re-interventions ( $10 \%$ vs. $74 \% ; p=.001$ ) compared with control fistulae. ${ }^{21}$

\section{Use of anastomotic devices}

The Optiflow (Bioconnect Systems, Ambler, PA, USA) is an internal anastomotic connecting device placed inside the artery and vein, with the purpose of improving hemodynamics and standardizing AVF creation. A non-randomized study in 41 patients and 39 matched control patients reported comparable unassisted maturation rates at 14,42 , and 90 days of $76 \%, 72 \%$, and $68 \%$ for the Optiflow group and $67 \%, 68 \%$, and $76 \%$ in the control group, respectively. In this study, there was a trend to earlier maturation in the Optiflow group compared with the control group $(p=.059){ }^{22,23}$ 
The VasQ (Laminate Medical Technologies, Israel) external metallic device creates a $60^{\circ}$ anastomotic angle between artery and vein to optimize hemodynamic conditions. A single center study of 20 patients reported primary patency rates of $95 \%, 79 \%$ and $79 \%$, with unassisted maturation rates of $80 \%, 79 \%$ and $74 \%$, at 1, 3, and 6 months of followup, respectively. At the end of the follow-up period, 14 of 15 patients requiring dialysis were able to use the AVF. ${ }^{24}$

Endovascular artery and vein diameter enhancement by balloon and probe angioplasty

In a retrospective study of primary intra-operative balloon angioplasty (PBA) of small cephalic veins in 62 AVFs (55 patients), 53 (85.4\%) remained patent and subsequently underwent balloon assisted maturation (BAM) with a resulting functional AVF. These consisted of 47 of the original AVFs and six new AVFs created at other sites. Occlusions occurred in 14 of the 55 fistulae (25.5\%), and seven were salvaged using BAM techniques. In another study, 40 patients, all candidates for distal AVF with a cephalic vein of $\leq 2 \mathrm{~mm}$, were randomized to two different surgical procedures: PBA of a long segment of the cephalic vein from the wrist up to the elbow $(n=19)$ versus hydrostatic dilatation (HD) of a short venous segment $(5 \mathrm{~cm})$ at the level of the anastomosis $(n=21)$. The mean fistula maturation time was 32 days in the PBA group and 55 days in the conventional group $(p=.04)$. The 6 month reintervention rate was significantly lower in the PBA group (5\%) compared with the conventional group (43\%) $(p=.02)$. At 6 months, primary patency rates were $95 \%$ in the PBA group and $57 \%$ in the other group $(p=.01) .^{25,26}$

Venous angioplasty with a probe was attempted in 93 patients with distal and proximal AVF creation. Early failure occurred in $20 \%$, and $70 \%$ of AVFs matured sufficiently for cannulation. Variables with significant impact on the FTM rate by univariate analysis were: body mass index $(p=.041)$, artery diameter $(p<.001)$, vein diameter $(p=.004)$, and vein diameter after dilatation $(p=.002)$. Multivariate analysis showed body mass index $(p=.038)$, artery diameter $(p=.001)$, and the diameter of the vein after dilatation $(p=.018)$ significantly affecting maturation. ${ }^{27}$ In another study PBA was performed during AVF creation, to treat arterial and venous stenosis diagnosed by a preliminary ultrasound examination. Sixty-nine AVFs in 58 patients were compared with 160 patients with nonstenosed vessels, receiving 188 AVFs without intra-operative PBA. Early thrombosis and FTM occurred in seven and eight patients in the PBA group and in 15 and 13 patients in the control group, respectively. Total failure occurred in 24\% of the PBA group and $14 \%$ of the controls $(p=.2)$. The primary patency rates at 6,12 , and 24 months were $78 \%, 72 \%$, and $59 \%$ for the PBA group and $85 \%, 78 \%$, and $78 \%$ for the control group $(p<.01)$. 
The secondary patency rates at 6,12 , and 24 months were $91 \%, 86 \%$, and $80 \%$ for the PBA group, and $95 \%, 95 \%$ and $92 \%$ for the controls $(p<.01){ }^{28}$

\section{Post-operative endovascular and surgical methods to improve arterio- venous fistula maturation}

\section{Endovascular techniques}

Percutaneous transluminal angioplasty (PTA) of venous outflow (VO) and JAS yielded a clinical success rate ranging from $43 \%$ to $97 \%$ with one year primary and secondary patencies of $28 \%$ to $72 \%$ and $68 \%$ to $96 \%$, respectively (Table 1 ). ${ }^{29-48}$ The best results were obtained when the juxta-anastomotic and efferent vein were dilated with 4-6 mm balloons. Rupture of the weak venous wall is common (15\% of patients), the majority of which can be managed with prolonged balloon inflation. Immature AVFs are ideally needled 7-14 days after successful dilation to allow hematoma caused by cannulation and local anesthesia to resorb. Results after dilation of diseased radial arteries feeding the RCAVF are better, with primary patency rates ranging from $65 \%$ to $83 \%$, and secondary patency rates of $86 \%$ to $96 \% .^{10}, 49$ Post-dilation arterial endothelial injuries might be less pronounced compared with veins, with a lower incidence of NIH and restenosis, resulting in more favorable patencies. ${ }^{50,51}$ The idea behind the potential benefit from paclitaxel coated balloon (PCB) use for non-matured access is to decelerate the progression of $\mathrm{NIH}$ formation and improve the treated lesion patency and thus AVF survival. The results from PCB studies have been promising with target lesion primary patency of $72 \%$ to $81 \%$ and $60 \%$ at 6 and 12 months, respectively. ${ }^{52}$

Balloon assisted maturation (BAM) is usually used to treat non-dilating veins and can be performed with conventional angiographic techniques or under ultrasound guidance. The clinical success rate ranges from $55 \%$ to $89 \% .^{53-56}$ In 336 office based BAM procedures, the most common complications were hematoma (40\%), extravasation or rupture $(9 \%)$, formation of puncture site hematoma (4\%), and thrombosis (2\%). A significant increase in complications was noted in BAM procedures performed in forearm AVFs vs. upper arm AVFs (67\% vs. 54\%) and with the use of large balloons compared with the use of small balloons (72\% vs. $52 \%) .^{57}$

Endovascular accessory vein obliteration (AVO) in 42 patients treated by 56 embolizations resulted in fistula maturation in 32 of 42 patients (76\%). Average time from AVO to fistula maturation was 38 days. Angioplasty in addition to AVO was found in a statistically higher percentage of patients with fistula success versus failure ( $31 \% \mathrm{vs}$. $8 \% ; p=.039$ ). Thirty-four patients underwent AVO without angioplasty, which resulted in 
successful fistula maturation in 22 cases $(65 \%){ }^{58}$ Stent graft placement in long segment stenosed veins may be the ultimate salvage technique for non-matured AVFs with an acceptable outcome (primary success rate 100\%; primary patency rate $65 \%$; secondary patency rate $72 \%){ }^{59}$

Table 1 Results of endovascular techniques to enhance arteriovenous fistula maturation.

\begin{tabular}{|c|c|c|c|c|c|}
\hline Author & Patients (n) & Intervention & CS & PP & SP \\
\hline Beathard et al. $1999^{29}$ & 63 & VO/AVO & 82 & & 75 \\
\hline Turmel-Rodrigues et al. $2001^{30}$ & 69 & JAS/VO & 97 & 39 & 79 \\
\hline Beathard et al. $2003^{31}$ & 100 & JAS/VO/AVO & 92 & & 68 \\
\hline Tordoir et al. $2003^{32}$ & 12 & JAS/VO & 43 & & \\
\hline Shin et al. $2005^{33}$ & 19 & JAS & 74 & 61 & 82 \\
\hline Falk $2006^{34}$ & 65 & JAS/VO & 74 & 64 & 68 \\
\hline Song et al. $2006^{35}$ & 22 & JAS/VO & 95 & 28 & 85 \\
\hline Asif et al. $2006^{36}$ & 41 & JAS & 93 & 46 & 94 \\
\hline Nassar et al. $2006^{37}$ & 119 & JAS/VO/AVO & 83 & 62 & 94 \\
\hline Barone et al. $2007^{38}$ & 43 & $\mathrm{Al} / \mathrm{JAS} / \mathrm{VO}$ & 85 & & \\
\hline Clark et al. $2007^{39}$ & 101 & JAS/VO & 88 & 34 & 72 \\
\hline Singh et al. $2008^{40}$ & 32 & JAS/VO/AVO & 78 & & \\
\hline Ascher et al. $2009^{53}$ & 27 & BAM & 89 & & \\
\hline Hong et al. $2009^{41}$ & 8 & JAS & 87 & 56 & 87 \\
\hline McLafferty et al. $2009^{42}$ & 23 & VO & 93 & & \\
\hline Raynaud et al. $2009^{49}$ & 25 & $\mathrm{Al}$ & 91 & 83 & 86 \\
\hline Turmel-Rodrigues et al. $2009^{10}$ & 74 & $\mathrm{Al}$ & 98 & 65 & 96 \\
\hline Natario et al. $2010^{43}$ & 30 & JAS & 97 & 37 & 96 \\
\hline Miller et al. $2011^{44}$ & 140 & VO/AVO & 79 & 38 & 69 \\
\hline Gallagher et al. $2012^{54}$ & 45 & BAM & 83 & & \\
\hline Han et al. $2013^{45}$ & 141 & JAS/VO & 96 & 72 & 83 \\
\hline DerDerian et al. $2013^{55}$ & 30 & BAM & 55 & & \\
\hline Ahmed et al. $2014^{58}$ & 42 & AVO & 76 & & \\
\hline Park et al. $2015^{46}$ & 24 & VO & 96 & 59 & \\
\hline Jeon et al. $2016^{47}$ & 59 & JAS/VO & 95 & 71 & \\
\hline Rizvi et al. $2017^{56}$ & 54 & BAM & 56 & & \\
\hline Park et al. $2017^{48}$ & 84 & JAS/VO/AVO & 94 & & 94 \\
\hline Bavare et al. $2017^{59}$ & 12 & VO/stent graft & 100 & 65 & 72 \\
\hline
\end{tabular}

CS: clinical success (\%); PP: 1 year primary patency (\%); SP: 1 year secondary patency (\%); JAS: juxtaanastomotic stenosis; Al: arterial inflow; VO: venous outflow; AVO: accessory vein obliteration; BAM: balloon assisted maturation. 


\section{Surgical techniques}

Proximal AV neo-anastomosis (PNA) and accessory vein ligation (AVL) are usually the techniques for intervention of non-matured fistulae (Table 2). A nonrandomized study in 46 patients of surgical (neo-anastomosis: 31) versus endovascular intervention (15) showed longer cumulative patency for the surgical group compared with the group that had angioplasty $(p=.05)$. One year cumulative patency was $83 \%$ and $40 \%$ for surgery vs. angioplasty, respectively. AVFs with surgical intervention for FTM had a similar one year cumulative patency to those AVFs that did not require intervention to promote maturation..$^{60}$ Similar results were found in a consecutive series of 73 patients with AVF non-maturation and JAS, treated either by surgery (21) or PTA (52). Surgery meant creation of a proximal neo-anastomosis excluding the area of stenosis. Anatomical and clinical success rates were $86 \%$ and $90 \%$ for surgery, and $75 \%$ and $92 \%$ for PTA. At 1 year, the primary patency rates were $71 \%$ for surgery and $41 \%$ for PTA, respectively $(p<.02)$. There was no significant difference between the two groups with respect to assisted primary patency (95\% vs. 92\%). These results suggest that the treatment of anastomotic stenoses should be surgical rather than endovascular. Angioplasty and surgery have shown similar results when used to treat other perianastomotic stenoses, but repeat procedures were more frequent with angioplasty. ${ }^{61}$ In 31 patients of a cohort of 163 AVFs, 40 endovascular and open surgical reinterventions were needed because of FTM. This resulted in an average of 1.29 interventions per non-maturing AVF. The success rate was $94 \%$ with a one year secondary patency of $85 \% .{ }^{62}$ Recently, a study of 80 patients with 81 revised RCAVFs by formation of a neo-anastomosis in the proximal cephalic vein segment, reported a primary patency of $78 \%, 69 \%$, and 55\%, at 12, 24, and 36 months, respectively. Failure to mature was the indication for revision in 50 fistulae, 17 were revised for poor flow, and 14 were revised for thrombosis. Compared with neoanastomoses that were performed for non-matured RCAVFs, those performed on the 31 matured fistulae exhibited better patency rates $(p=.04) .{ }^{63}$ A group of 63 patients with non-matured RCAVFs (56) and BCAVFs (7) had angiography with subsequent PTA (24) and accessory vein ligation (39). Clinical success rate was $82 \%$ and $83 \%$ for AVL and PTA, respectively, with a one year fistula patency of $75 \% .{ }^{29}$ In another retrospective study, 17 patients underwent ligation of accessory veins of poorly developed AVFs. There were 14 RC- and three BCAVFs. After identifying accessory veins with a fistulagram, nonabsorbable sutures were placed percutaneously around each accessory vein in proximity to the AVF. Of 17 AVFs, 15 (88\%) matured successfully after the procedure. The average number of accessory veins ligated was 1.7 (range 1-3). ${ }^{64}$ In a prospective study, contrast enhanced magnetic resonance angiography (CE-MRA) was performed preoperatively for the determination of vessel diameters, stenoses, and occlusions. The location and caliber 
of accessory veins was determined. Non-maturation occurred in 10 (67\%) of 15 RCAVFs. Large caliber accessory veins (4), venous stenosis (3), or both (2) were associated with AVF non-maturation. The presence of large caliber accessory veins was the only significant predictor of nonmaturation $(p=.01)$. Preoperatively detected accessory veins with a diameter $>70 \%$ of the cephalic vein diameter had a sensitivity, specificity, positive predictive value (PPV), and negative predictive value (NPV) of $80 \%, 100 \%, 100 \%$ and $91 \%$ for prediction of AVF non-maturation. Accessory vein ligation and dilatation of venous stenoses resulted in an overall AVF salvage rate of $89 \% .{ }^{65}$ Engstrom et al. ${ }^{66}$ reviewed fistulagrams of non-maturing AVFs in 145 patients for the presence of accessory veins. In 49 (34\%) a stenosis without any accessory veins was shown, $76(52 \%)$ had a stenosis with one or more accessory veins, and 20 (14\%) had an accessory vein without concurrent stenosis. Eighteen AV fistulae had one or more accessory veins without coexisting stenosis. Coil embolization of dominant accessory veins with a caliber decrease immediately downstream resulted in a $100 \%$ maturation rate versus $67 \%$ for fistulae without this configuration. Accessory vein size was not correlated with maturation rates. The majority of non-maturing fistulae with accessory veins had a coexisting stenosis.

Table 2 Results of surgical techniques to enhance arteriovenous fistula maturation.

\begin{tabular}{|c|c|c|c|c|c|}
\hline Author & Patients (n) & Intervention & CS & PP & SP \\
\hline Beathard et al. $1999^{29}$ & 39 & AVL & 82 & & 75 \\
\hline Faiyaz et al. $2002^{64}$ & 17 & AVL & 88 & & \\
\hline Planken et al. $2007^{65}$ & 10 & AVL & 89 & & \\
\hline Mallik et al. $2011^{63}$ & 50 & PNA & 72 & 72 & 87 \\
\hline Long et al. $2011^{61}$ & 21 & PNA & 90 & 71 & 95 \\
\hline Bharat et al. $2012^{19}$ & 54 & SLOT & 83 & & \\
\hline Lee et al. $2013^{60}$ & 31 & PNA/AVL & 87 & & 86 \\
\hline Mufty et al. $2015^{62}$ & 31 & PNA/AVL & 94 & 68 & 85 \\
\hline Nikam et al. $2015^{23}$ & 41 & Optiflow & 78 & $78^{*}$ & \\
\hline Sadaghianloo et al. $2016^{21}$ & 53 & RADAR & 92 & $93^{\$}$ & $100^{\$}$ \\
\hline Chemla et al. $2016^{24}$ & 20 & Laminate & 74 & $79^{\$}$ & \\
\hline Darcy et al. $2017^{20}$ & 342 & SLOT & 86 & 43 & 82 \\
\hline
\end{tabular}

CS: clinical success (\%); PP: 1 year primary patency (\%); SP: 1 year secondary patency (\%); PNA: proximal neoanastomosis; AVL: accessory vein ligation; SLOT: straight line onlay technique; RADAR: radial artery deviation and reimplantation; Optiflow: internal anastomotic device; Laminate: external anastomotic device. * at 3 months, \$ at 6 months.

The importance of optimal operative conditions with vessel dilation has been stressed in a randomized study on the use of regional anesthesia with brachial plexus block for AVF creation in 126 patients. In this study, primary patency at 3 months was $84 \%$ for patients 
having plexus block versus $62 \%$ for patients having local anesthesia. Immediate patency was higher with regional anesthesia (93\% vs. 73\%), as was functional patency at 3 months in RCAVFs (73\% vs. $40 \%) .{ }^{67} \mathrm{~A}$ recent meta-analysis confirmed sympathetic blockade with regional brachial plexus block of sustained benefit in patients undergoing AVF formation. ${ }^{68}$

\section{DISCUSSION}

Failure to mature of autologous arteriovenous fistulae is a major issue in the creation of functional hemodialysis vascular access. The current recommendation is to closely monitor newly created fistulae and if maturation is not sufficient, to intervene to enhance AVF function. Both surgical and endovascular techniques are at the disposal of the physician involved in vascular access management; however, from the literature it is unclear whether one method is to be preferred over the other and which short and long term outcomes can be expected.

Conventional fluoroscopy guided percutaneous transluminal angioplasty is usually performed for perianastomotic and efferent vein stenosis and initial clinical success rates are reported to be in the range of $43 \%$ to $97 \%$. The published studies evaluating longterm outcomes in AVFs requiring interventions to promote maturation report primary patency rates ranging from $28 \%$ to $72 \%$ and secondary patency rates of $68 \%$ to $96 \%$ at one year.

The tradeoffs from aggressive efforts to maximize AVF use may be prolonged catheter dependence and a high frequency of catheter related bacteremia. AVFs that require intervention before maturation have shorter secondary patencies than those that mature without an intervention and have comparable patencies with AVGs that are used successfully without a prior intervention..$^{69}$ The cumulative fistula patency is significantly inferior in patients requiring two or more interventions to achieve AVF maturation, as compared with those requiring zero or one intervention. Moreover, AVFs requiring two or more interventions to promote maturation need more interventions to maintain long-term patency after dialysis use..$^{51}$ Randomized studies comparing BAM with conventional interventional methods for AVF maturation are desperately needed. In addition, identifying patients who do not require repeated angioplasties following successful BAM might be helpful. A detailed survey of both clinical and biological predictors in patients being treated by BAM may support decision making. For example, it is possible that patients with good endothelial function (large increase in diameter with flow mediated dilatation) and small vessels, comprise the particular subset that would benefit from BAM..$^{70,71}$ 
The type of vascular intervention for FTM may affect clinical success and long-term fistula patency. The vessel injury resulting from angioplasty is usually greater than that obtained by surgical revision. ${ }^{54,60}$ The few studies on endovascular versus surgical intervention show better outcome for the surgical group, in particularly for primary patency which ranges from $68 \%$ to $94 \%$ compared with a dismal $28 \%$ to $72 \%$ patency in endovascularly treated patients. In the latter group repeated PTA is needed to achieve a $68 \%$ to $97 \%$ AVF cumulative survival rate after 1 year of follow-up. Selection bias may be partly responsible for this difference; a preference for surgical repair of juxtaanastomotic stenoses is most likely the case, while an endovascular approach for treatment of venous outflow stenoses will probably be chosen. Surgical or endovascular accessory vein ligation/occlusion as a single treatment option was promoted more than a decade ago, but currently this is usually performed as an additional procedure to other treatment modalities. PTA in combination with endovascular accessory vein obliteration or surgical accessory vein ligation has been reported in a few studies with reasonable success. The rationale to occlude accessory veins, ligate the medial cubital vein, or band the mainstream vein continues to be debated and is of no proven value at the moment. Surgical modification of the AV anastomosis has been proposed to optimise haemodynamic conditions in terms of lowering vessel wall shear stress (WSS) and improving outward remodeling. Computational simulation models show an advantage in terms of lower WSS with a $30^{\circ}$ angle AV anastomosis compared with a more smooth anastomosis of $60^{\circ}$ and $90^{\circ} .{ }^{72}$ The SLOT anastomotic technique is based on this concept and has a significantly better outcome compared with conventional techniques (functional AVF: $83 \%$ vs. $56 \%$ ). However, these results have been accomplished in a single center only and randomized studies are lacking. Conversely, a recent study shows different results with more juxta-anastomotic stenoses in AVFs created with an acute angle $\left(30^{\circ}\right)$, compared with AVFs with a smooth angle anastomosis $\left(60-90^{\circ}\right) .^{73}$ In this respect, standardizing the anastomotic angle with internal and external devices is another way of improving hemodynamics. Small patient studies show similar early FTM rates compared with conventional techniques, and lack long-term outcomes. The place of these devices for enhancement of fistula maturation remains unclear. Although the RADAR technique is reported to have improved maturation rates with a greater number of functional fistulae, one might argue whether ligation of the radial artery is desirable, in particular when collateral flow to the hand, for instance in diabetic patients, may be insufficient.

Vascular access surgical procedures are particularly prone to vessel spasm, because of local handling of vessels and enhanced sympathetic nervous system activity, in turn resulting in impaired blood flow. In particular, in ESRD patients this might be an issue because of the underlying arterial calcification and/or narrowing, often compounded by 
diabetes mellitus and hypertension. One of the major determinants of successful AVF creation is adequacy of blood flow in the perioperative period. In this respect, intraoperative arterial and vein diameter enhancement with balloons and probes has been advocated with reasonably good results (70-100\% maturation) and a $96 \%$ patency at six months. ${ }^{25-28}$

The major limitation of the present study is lack of a formal systematic review and metaanalysis. Failure to mature is an important yet difficult to tackle, deleterious outcome of AVF surgery, resulting in many different efforts to treat or mitigate the risk of FTM. Although all these efforts in themselves are commendable, this does hamper a formal comparison by means of a systematic review and meta-analysis, especially as there is no gold standard. Therefore, a narrative review of the available evidence was selected. Given the methodological strengths and limitations of the literature reviewed above, all results appear more or less comparable. Therefore, it is concluded that the treatment of FTM can be left to the discretion of the treating physician in connection with the locally available facilities.

\section{SUMMARY}

Aggressive intervention for maturation failure has been advocated to treat early complications of autologous arteriovenous fistulae. Endovascular means are the first line of treatment in particular in arterial and venous stenoses. Primary surgical intervention with new proximal neoanastomosis for juxta-anastomotic stenoses has a better primary success rate with good long-term patencies compared with endovascular methods. In addition, attempts to optimize perioperative hemodynamic conditions and surgical technique modifications are worthwhile to improve fistula outcome and have the potential to increase the number of functional AVFs.

\section{CONFLICTS OF INTEREST}

None.

\section{FUNDING}

None. 


\section{REFERENCES}

1. Murad MH, Elamin MB, Sidawy AN, et al. Autogenous versus prosthetic vascular access for hemodialysis: a systematic review and meta-analysis. Journal of vascular surgery. 2008;48(5 Suppl):34S-47S.

2. Almasri J, Alsawas $M$, Mainou $M$, et al. Outcomes of vascular access for hemodialysis: A systematic review and meta-analysis. Journal of vascular surgery. 2016;64(1):236-43.

3. Leermakers JJ, Bode AS, Vaidya A, et al. Cost-effectiveness of vascular access for haemodialysis: arteriovenous fistulas versus arteriovenous grafts. European journal of vascular and endovascular surgery. 2013;45(1):84-92.

4. Hicks CW, Canner JK, Arhuidese I, et al. Mortality benefits of different hemodialysis access types are age dependent. Journal of vascular surgery. 2015;61(2):449-56.

5. Lok CE, Allon M, Moist $L$, et al. Risk equation determining unsuccessful cannulation events and failure to maturation in arteriovenous fistulas (REDUCE FTM I). Journal of the American Society of Nephrology. 2006;17(11):3204-12.

6. Dember LM, Beck GJ, Allon M, et al. Effect of clopidogrel on early failure of arteriovenous fistulas for hemodialysis: a randomized controlled trial. Jama. 2008;299(18):2164-71.

7. Wilmink T, Hollingworth L, Powers S, et al. Natural History of Common Autologous Arteriovenous Fistulae: Consequences for Planning of Dialysis Access. European Journal of Vascular and Endovascular Surgery. 2016;51(1):134-40.

8. Masengu A, Maxwell AP, Hanko JB. Investigating clinical predictors of arteriovenous fistula functional patency in a European cohort. Clinical kidney journal. 2016;9(1):142-7.

9. Allon M, Greene T, Dember LM, et al. Association between Preoperative Vascular Function and Postoperative Arteriovenous Fistula Development. Journal of the American Society of Nephrology. 2016;27(12):3788-95.

10. Turmel-Rodrigues L, Boutin JM, Camiade $C$, et al. Percutaneous dilation of the radial artery in nonmaturing autogenous radial-cephalic fistulas for haemodialysis. Nephrology, dialysis, transplantation. 2009;24(12):3782-8.

11. Bylsma LC, Gage SM, Reichert H, et al. Arteriovenous Fistulae for Haemodialysis: A Systematic Review and Meta-analysis of Efficacy and Safety Outcomes. European journal of vascular and endovascular surgery. 2017;54(4):513-22.

12. Allon $\mathrm{M}$, Robbin $\mathrm{ML}$, Umphrey HR, et al. Preoperative arterial microcalcification and clinical outcomes of arteriovenous fistulas for hemodialysis. American journal of kidney diseases. 2015;66(1):84-90.

13. Voormolen EHJ, Jahrome AK, Bartels LW, et al. Nonmaturation of arm arteriovenous fistulas for hemodialysis access: A systematic review of risk factors and results of early treatment. Journal of vascular surgery. 2009;49(5):1325-36.

14. Zeebregts CJ, van den Dungen JJ, van Det RJ, et al. Randomized clinical trial of continuous sutures or non-penetrating clips for radiocephalic arteriovenous fistula. The British journal of surgery. 2004;91(11):1438-42. 
15. Lin $\mathrm{PH}$, Bush RL, Nelson JC, et al. A prospective evaluation of interrupted nitinol surgical clips in arteriovenous fistula for hemodialysis. American journal of surgery. 2003;186(6):625-30.

16. Nguyen KP, Teruya T, Alabi O, et al. Comparison of Nonpenetrating Titanium Clips versus Continuous Polypropylene Suture in Dialysis Access Creation. Ann Vasc Surg. 2016;32:15-9.

17. Ross JR. Creation of native arteriovenous fistulas with interrupted anastomoses using a self-closing clip device - one clinic's experience. The journal of vascular access. 2002;3(4):140-6.

18. Aitken $\mathrm{E}$, Jeans $\mathrm{E}$, Aitken $\mathrm{M}$, et al. A randomized controlled trial of interrupted versus continuous suturing techniques for radiocephalic fistulas. Journal of vascular surgery. 2015;62(6):1575-82.

19. Bharat $A$, Jaenicke $M$, Shenoy S. A novel technique of vascular anastomosis to prevent juxta-anastomotic stenosis following arteriovenous fistula creation. Journal of vascular surgery. 2012;55(1):274-80.

20. Darcy $M$, Vachharajani N, Zhang $T$, et al. Long-term outcome of upper extremity arteriovenous fistula using pSLOT: single-center longitudinal follow-up using a protocol-based approach. The journal of vascular access. 2017:0.

21. Sadaghianloo N, Declemy S, Jean-Baptiste E, et al. Radial artery deviation and reimplantation inhibits venous juxta-anastomotic stenosis and increases primary patency of radial-cephalic fistulas for hemodialysis. Journal of vascular surgery. 2016;64(3):698-706 e1.

22. Chemla $E$, Tavakoli A, Nikam M, et al. Arteriovenous fistula creation using the Optiflow vascular anastomotic connector: the OPEN (Optiflow PatEncy and MaturatioN) study. The journal of vascular access. 2014;15(1):38-44.

23. Nikam M, Chemla ES, Evans J, et al. Prospective controlled pilot study of arteriovenous fistula placement using the novel Optiflow device. Journal of vascular surgery. 2015;61(4):1020-5.

24. Chemla E, Velazquez CC, D'Abate F, et al. Arteriovenous fistula construction with the VasQ external support device: a pilot study. The journal of vascular access. 2016;17(3):243-8.

25. De Marco Garcia LP, Davila-Santini LR, Feng Q, et al. Primary balloon angioplasty plus balloon angioplasty maturation to upgrade small-caliber veins $(<3 \mathrm{~mm})$ for arteriovenous fistulas. Journal of vascular surgery. 2010;52(1):139-44.

26. Veroux $P$, Giaquinta $A$, Tallarita $T$, et al. Primary balloon angioplasty of small $(</=2$ $\mathrm{mm}$ ) cephalic veins improves primary patency of arteriovenous fistulae and decreases reintervention rates. Journal of vascular surgery. 2013;57(1):131-6.

27. Fila B, Lovcic V, Sonicki Z, et al. Vein diameter after intraoperative dilatation with vessel probes as a predictor of success of hemodialysis arteriovenous fistulas. Medical science monitor : international medical journal of experimental and clinical research. 2014;20:191-8.

28. Napoli $M$, Lefons $M L$, Mangione $D$, et al. Primary intraoperative transluminal angioplasty: a new approach to reduce the early failure of distal arteriovenous fistulas. The journal of vascular access. 2015;16(3):250-4. 
29. Beathard GA, Settle SM, Shields MW. Salvage of the nonfunctioning arteriovenous fistula. American journal of kidney diseases. 1999;33(5):910-6.

30. Turmel-Rodrigues L, Mouton A, Birmele B, et al. Salvage of immature forearm fistulas for haemodialysis by interventional radiology. Nephrology, dialysis, transplantation. 2001;16(12):2365-71.

31. Beathard GA, Arnold P, Jackson J, et al. Aggressive treatment of early fistula failure. Kidney international. 2003;64(4):1487-94.

32. Tordoir JH, Rooyens $\mathrm{P}$, Dammers $\mathrm{R}$, et al. Prospective evaluation of failure modes in autogenous radiocephalic wrist access for haemodialysis. Nephrology, dialysis, transplantation. 2003;18(2):378-83.

33. Shin SW, Do YS, Choo SW, et al. Salvage of immature arteriovenous fistulas with percutaneous transluminal angioplasty. Cardiovasc Intervent Radiol. 2005;28(4):434-8.

34. Falk A. Maintenance and salvage of arteriovenous fistulas. J Vasc Interv Radiol. 2006;17(5):807-13.

35. Song HH, Won YD, Kim YO, et al. Salvaging and maintaining non-maturing BresciaCimino haemodialysis fistulae by percutaneous intervention. Clinical radiology. 2006;61(5):404-9.

36. Asif $A$, Lenz $O$, Merrill $D$, et al. Percutaneous management of perianastomotic stenosis in arteriovenous fistulae: results of a prospective study. Kidney international. 2006;69(10):1904-9.

37. Nassar GM, Nguyen B, Rhee E, et al. Endovascular treatment of the "failing to mature" arteriovenous fistula. Clinical journal of the American Society of Nephrology. 2006;1(2):275-80.

38. Barone GW, Wright CF, Krause MW, et al. Hemodialysis access success: beyond the operating room. American journal of surgery. 2007;194(5):668-71.

39. Clark TW, Cohen RA, Kwak A, et al. Salvage of nonmaturing native fistulas by using angioplasty. Radiology. 2007;242(1):286-92.

40. Singh $P$, Robbin $M L$, Lockhart $M E$, et al. Clinically immature arteriovenous hemodialysis fistulas: effect of US on salvage. Radiology. 2008;246(1):299-305.

41. Hong HP, Kim SK. Usefulness of percutaneous intervention with transarterial approach in the salvage of nonmaturing native fistulas status-post transvenous approach failure: transarterial approach in the salvage of nonmaturing native fistulas. Cardiovasc Intervent Radiol. 2009;32(6):1252-6.

42. McLafferty RB. Techniques to enhance arteriovenous fistula maturation. Perspectives in vascular surgery and endovascular therapy. 2009;21(1):41-5.

43. Natario A, Turmel-Rodrigues $L$, Fodil-Cherif $M$, et al. Endovascular treatment of immature, dysfunctional and thrombosed forearm autogenous ulnar-basilic and radial-basilic fistulas for haemodialysis. Nephrology, dialysis, transplantation. 2010;25(2):532-8.

44. Miller GA, Hwang W, Preddie D, et al. Percutaneous salvage of thrombosed immature arteriovenous fistulas. Seminars in dialysis. 2011;24(1):107-14.

45. Han M, Kim JD, Bae Jl, et al. Endovascular treatment for immature autogenous arteriovenous fistula. Clinical radiology. 2013;68(6):e309-15. 
46. Park JY, Yoo CH. On Postoperative Day Balloon Angioplasty for Salvage of NewlyPlaced, Flow-Limiting Native Arteriovenous Fistula. Vasc Specialist Int. 2015;31(1):20-4.

47. Jeon EY, Cho YK, Cho SB, et al. Predicting Factors for Successful Maturation of Autogenous Haemodialysis Fistulas After Salvage Percutaneous Transluminal Angioplasty in Diabetic Nephropathy: A Study on Follow-Up Doppler Ultrasonography. Iranian journal of radiology. 2016;13(1):e32559.

48. Park HS, Lee YH, Kim HW, et al. Usefulness of assisted procedures for arteriovenous fistula maturation without compromising access patency. Hemodialysis international International Symposium on Home Hemodialysis. 2017;21(3):335-42.

49. Raynaud A, Novelli L, Bourquelot $P$, et al. Low-flow maturation failure of distal accesses: Treatment by angioplasty of forearm arteries. Journal of vascular surgery. 2009;49(4):995-9.

50. Alp NJ, West NE, Arnold N, et al. Increased intimal hyperplasia in experimental vein graft stenting compared to arterial stenting: comparisons in a new rabbit model of stent injury. Cardiovascular research. 2002;56(1):164-72.

51. Lee $T$, Ullah $A$, Allon $M$, et al. Decreased cumulative access survival in arteriovenous fistulas requiring interventions to promote maturation. Clinical journal of the American Society of Nephrology. 2011;6(3):575-81.

52. Karnabatidis D, Kitrou P. Drug eluting balloons for resistant arteriovenous dialysis access stenosis. The journal of vascular access. 2017;18(Suppl. 1):88-91.

53. Ascher E, Hingorani A, Marks N. Duplex-guided balloon angioplasty of failing or nonmaturing arterio-venous fistulae for hemodialysis: a new office-based procedure. Journal of vascular surgery. 2009;50(3):594-9.

54. Gallagher JJ, Boniscavage $\mathrm{P}$, Ascher $\mathrm{E}$, et al. Clinical experience with office-based duplex-guided balloon-assisted maturation of arteriovenous fistulas for hemodialysis. Ann Vasc Surg. 2012;26(7):982-4.

55. DerDerian T, Hingorani A, Ascher E, et al. To BAM or not to BAM?: A closer look at balloon-assisted maturation. Ann Vasc Surg. 2013;27(1):104-9.

56. Rizvi SA, Usoh F, Hingorani A, et al. The Clinical Efficacy of Balloon-Assisted Maturation of Autogenous Arteriovenous Fistulae. Ann Vasc Surg. 2017;41:41-5.

57. DerDerian $T$, Hingorani $A$, Boniviscage $P$, et al. Acute complications after balloonassisted maturation. Ann Vasc Surg. 2014;28(5):1275-9.

58. Ahmed $\mathrm{O}$, Patel $M$, Ginsburg $M$, et al. Effectiveness of collateral vein embolization for salvage of immature native arteriovenous fistulas. J Vasc Interv Radiol. 2014;25(12):1890-4.

59. Bavare CS, Street TK, Peden EK, et al. Stent Grafts Can Convert Unusable Upper Arm Arteriovenous Fistulas into a Functioning Hemodialysis Access: A Retrospective Case Series. Front Surg. 2017;4:13.

60. Lee T, Tindni A, Roy-Chaudhury P. Improved cumulative survival in fistulas requiring surgical interventions to promote fistula maturation compared with endovascular interventions. Seminars in dialysis. 2013;26(1):85-9.

61. Long B, Brichart N, Lermusiaux $P$, et al. Management of perianastomotic stenosis of direct wrist autogenous radial-cephalic arteriovenous accesses for dialysis. Journal of vascular surgery. 2011;53(1):108-14. 
62. Mufty $\mathrm{H}$, Claes $\mathrm{K}$, Heye $\mathrm{S}$, et al. Proactive surveillance approach to guarantee a functional arteriovenous fistula at first dialysis is worth. The journal of vascular access. 2015;16(3):183-8.

63. Mallik M, Sivaprakasam R, Pettigrew GJ, et al. Operative salvage of radiocephalic arteriovenous fistulas by formation of a proximal neoanastomosis. Journal of vascular surgery. 2011;54(1):168-73.

64. Faiyaz R, Abreo K, Zaman F, et al. Salvage of poorly developed arteriovenous fistulae with percutaneous ligation of accessory veins. American journal of kidney diseases. 2002;39(4):824-7.

65. Planken RN, Duijm LE, Kessels AG, et al. Accessory veins and radial-cephalic arteriovenous fistula non-maturation: a prospective analysis using contrastenhanced magnetic resonance angiography. The journal of vascular access. 2007;8(4):281-6.

66. Engstrom Bl, Grimm LJ, Ronald J, et al. Accessory veins in nonmaturing autogenous arteriovenous fistulae: analysis of anatomic features and impact on fistula maturation. Seminars in dialysis. 2015;28(3):E30-4.

67. Aitken $E$, Jackson A, Kearns $R$, et al. Effect of regional versus local anaesthesia on outcome after arteriovenous fistula creation: a randomised controlled trial. Lancet. 2016;388(10049):1067-74.

68. Cerneviciute R, Sahebally SM, Ahmed K, et al. Regional Versus Local Anaesthesia for Haemodialysis Arteriovenous Fistula Formation: A Systematic Review and MetaAnalysis. European journal of vascular and endovascular surgery. 2017;53(5):734-42.

69. Harms JC, Rangarajan S, Young CJ, et al. Outcomes of arteriovenous fistulas and grafts with or without intervention before successful use. Journal of vascular surgery. 2016;64(1):155-62.

70. Roy-Chaudhury P, Lee $T$, Woodle B, et al. Balloon-assisted maturation (BAM) of the arteriovenous fistula: the good, the bad, and the ugly. Seminars in nephrology. 2012;32(6):558-63.

71. Shenoy S, Wood M, Matson S. Interventions for failed wrist fistulae: Is it worthwhile? The journal of vascular access. 2016;17 Suppl 1:S1-5.

72. Ene-lordache B, Cattaneo L, Dubini G, et al. Effect of anastomosis angle on the localization of disturbed flow in 'side-to-end' fistulae for haemodialysis access. Nephrology, dialysis, transplantation. 2013;28(4):997-1005.

73. Sadaghianloo N, Jean-Baptiste $E$, Rajhi $K$, et al. Increased reintervention in radialcephalic arteriovenous fistulas with anastomotic angles of less than 30 degrees. Journal of vascular surgery. 2015;62(6):1583-9 
SURGICAL AND ENDOVASCULAR INTERVENTIONS FOR FTM | $\mathbf{1 6 3}$ 



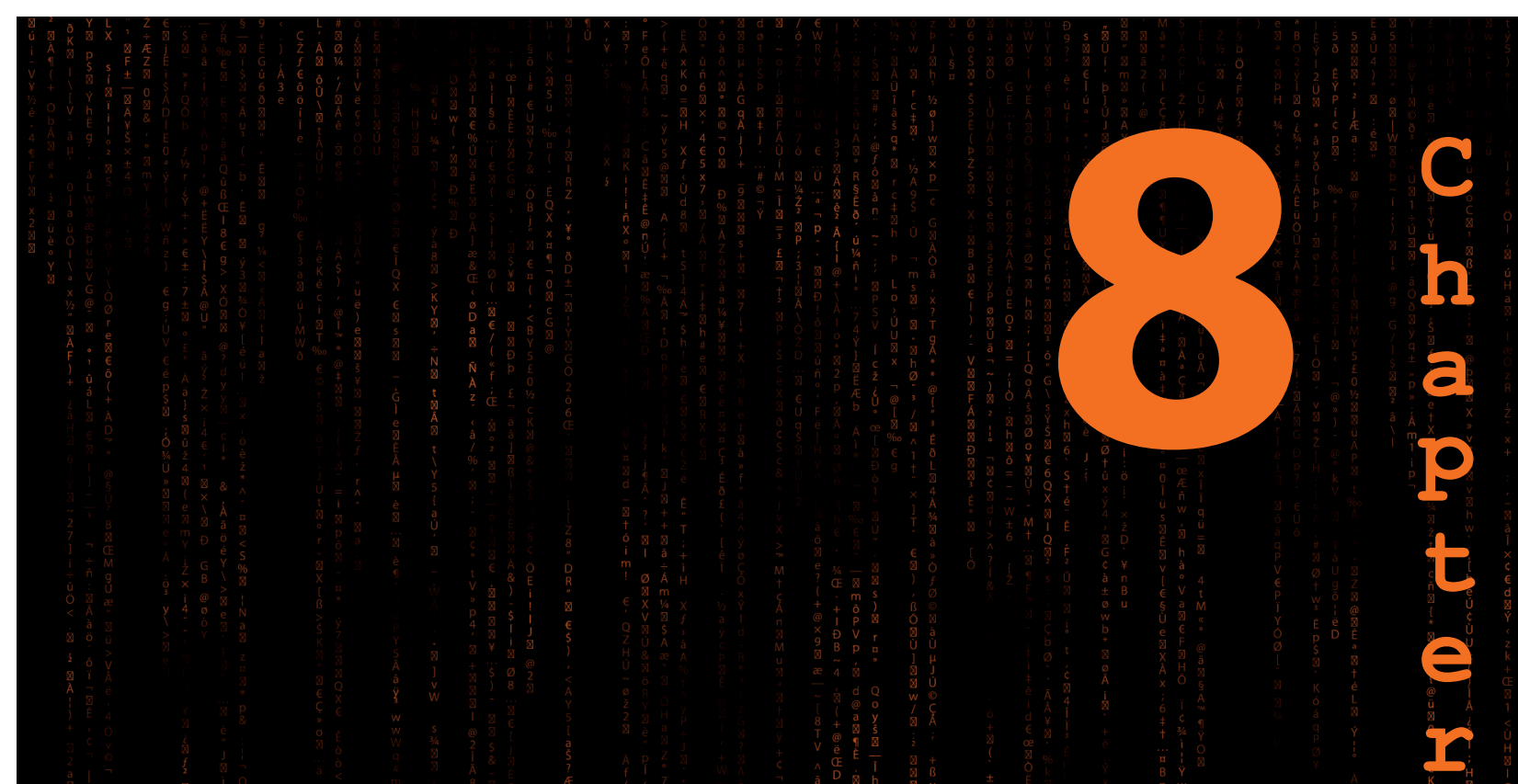

General Discussion 
166 | CHAPTER 8 
There are many challenges in arteriovenous fistula surgery, of which the most important one is to create an access that will successfully mature and can be cannulated for hemodialysis treatment. Failure to mature (FTM) has long been - and still is - considered a pressing issue in AVF creation.

The AVF is considered the vascular access of choice over arteriovenous grafts (AVGs) or central venous catheters (CVCs) for its better patency and lower morbidity and mortality rates., 2 In 2003 the Fistula First Breakthrough Initiative was coined to enhance the number of AVFs - especially in the United States where many patients commence hemodialysis via AVGs and CVCs. ${ }^{3}$ Although an increased use of AVFs was observed after the Fistula First Breakthrough Initiative, the clinical challenge of maturation was emphasized as well. ${ }^{4}$

Nowadays there is a shift from fistula first to fistula first, and catheter last, which is importantly due to relative high FTM rates and an incomplete understanding of AVF maturation. ${ }^{5-7}$ This incomplete understanding of maturation hampers clinical decisionmaking. ${ }^{8}$ Woo et al. for example asked a panel of 11 vascular access experts (based on peer recommendations) to rate 2088 AVF clinical scenarios with varying patient characteristics (e.g. age, vein size, diabetes). ${ }^{9}$ In only $74 \%$ the panelists reached agreement, highlighting the difficulties of decision-making in an incompletely understood clinical challenge.

The main aim of this thesis is to add to the understanding of AVF maturation, in an effort to answer to the call of the right access for the right patient. ${ }^{9,10}$ The main objective in this endeavor is to assess if the new and innovative approach of providing the additional information of patient-specific flow predictions in surgical planning can improve maturation rates. Previous attempts such as anastomosis adaptations, ${ }^{11}{ }^{12}$ anastomotic implants, ${ }^{13,14}$ and risk prediction models ${ }^{15}$ were promising at first, but no single approach to date has resulted in substantial improvements in AVF maturation rates. The computational model, which is evaluated in this thesis, is different from previous attempts as it is based on physics, can be individualized, and predicts immediate postoperative flow - a metric that unequivocally is related to AVF maturation. ${ }^{16-18}$

\section{ARTERIOVENOUS FISTULAE FOR HEMODIALYSIS}

In Chapter 1 the two main themes - AVFs for hemodialysis and patient-specific computational modeling - of this thesis were introduced and discussed in detail. When patients reach the end-stage of renal disease, they become dependent on renal replacement therapy, of which hemodialysis is the most predominant modality. A wellfunctioning vascular access (VA) is indispensable to connect the patient with the artificial 
kidney (i.e. dialyzer), and therewith provide adequate hemodialysis treatment. The AVF is the preferred vascular access, and is surgically created by connecting an artery with a superficial vein. Consequently, the distal peripheral resistance is bypassed and blood flow will increase significantly, initiating a remodeling process of the vein that ultimately results in a functional AVF. This remodeling process is called maturation and basically consists of three changes over the course of weeks to months. Blood volume flow increases in response to the low resistance conduit, venous diameters increase in response to the flow induced increased wall shear stress and the vessel wall thickens in response to the increased circumferential stress due to the increased transmural pressure. Obviously, this process is more complicated, since many inter- and intracellular processes are involved to orchestrate these changes and the exact trigger is still unkown. ${ }^{19-21}$

There are three main locations at which the anastomosis between the artery and vein usually is created: at the wrist, elbow and upper arm. In the forearm the vessels have smaller calibers and therewith higher resistance, in comparison to elbow and upper arm AVFs. As a result, forearm AVFs may have lower access flows, and an increased risk of failure to mature (FTM). In FTM the alterations described above occur insufficiently or not at all. Conversely, upper arm AVFs usually have high access flows with a potential risk of distal hypoperfusion resulting in ischemic complications. FTM occurs in $20-40 \%$ of all created AVFs, ${ }^{22-24}$ whereas approximately $20 \%$ of the patients experience various complaints of low distal perfusion. ${ }^{25,}{ }^{26}$ FTM is a major problem since many patients in (urgent) need for a permanent vascular access, may depend (longer) on a central venous catheter with its consequences of infection and subsequently higher mortality rates. ${ }^{27}$ In addition, a failing AVF usually requires multiple interventions to enhance maturation, or needs to be replaced with a new AVF. This undesirable situation puts an increased pressure on valuable health care resources, but most importantly increases patient burden.

To alleviate the challenge of FTM, a patient-specific model was developed that predicts immediate postoperative flows for the three conventional AVF configurations. ${ }^{28}$ The patient-specific model is a so-called pulse wave propagation model (PWPM) that describes how pressure and flow pulses travel along the arterial tree. ${ }^{29}$ The anatomy of interest is segmented, and each segment describes the local relation between pressure and flow via physical parameters as resistance, compliance, and inertia, which is based on the physical balance laws of mass and momentum. Each segment can be individualized by diameter measurements at the corresponding anatomical location within a patient, and flow measurements are used to estimate the peripheral resistance. Within a European collaboration (i.e. ARCH project) the model was developed by completing the necessary steps to make the model suitable for clinical decision- 
support. $^{30}$ Model development, verification, and implementation, together with mechanical characterization were completed in the pre-clinical phase. Of the clinical phase of model development, clinical workflow analysis, model optimization, personalization, and corroboration were completed. It was found that the model can reliably predict postoperative flow, and consequently it was concluded that this model could be useful in decision-support for AVF surgery. ${ }^{31-33}$

\section{MODELING STRATEGIES IN ARTERIOVENOUS FISTULA MATURATION AND FUNCTIONING}

In Chapter 2 complexity of AVF maturation is discussed, and how several modeling strategies - in particular patient-specific computational modeling - can be of benefit. AVF maturation is a complex interplay of many interrelated factors that to date is still incompletely understood. Maturation requires the vessel to remodel outwardly, and AVF functioning thereafter depends on the prevention of neointimal hyperplasia $(\mathrm{NIH})$ formation. ${ }^{19,34}$ The balance between the favorable outward remodeling and detrimental $\mathrm{NIH}$ formation can be disrupted by various factors, which usually are classified as up- and downstream events. ${ }^{20,35}$ Upstream events are the factors that cause endothelial and smooth muscle cell injury, that in turn set the downstream events in motion. Downstream events cause NIH by a complex interplay of cells, cytokines, and other signaling cascades.

In both events, hemodynamical factors such as flow and wall shear stress (WSS) are presumed to play important roles. Wall shear stress is the frictional force that is exerted on the vessel wall by flowing blood, and sensed by endothelial cells by cytoskeleton deformations. If for a given diameter the flow increases, WSS increases as well, and the cytoskeleton becomes more deformed. In response, several intra- and intercellular processes are initiated to relax vascular smooth muscle cells. ${ }^{36}$ Consequentially, vessel diameters increase and WSS decreases back to the normal physiological range.

After AVF creation, flow increases tremendously, and the vessels are consequently exposed to unphysiologically high levels of WSS. Nevertheless, over the course of weeks to months a new, almost physiological (i.e. without an AVF) equilibrium is reached. It has been shown that peak WSS levels almost entirely return to normal, but the mean WSS remains elevated. ${ }^{37}$ The latter is mainly explained by the end-diastolic flow in case of AVFs. Consequently, it has been hypothesized that peak rather than mean WSS is the primary driving force of AVF maturation. ${ }^{38}$

Despite the well-known and recognized importance of hemodynamical factors, many studies focus on patient characteristics that are a proxy for vascular health: peripheral 
arterial disease, cardio- and cerebrovascular disease, hypertension, diabetes, et cetera. All these factors share one common trait: they oversimplify complex and multifactorial diseases, and are then (separately) used to predict the outcome of the complex and multifactorial process of AVF maturation. Conceivably, it is no surprise that maturation is still incompletely understood.

We therefore argue that there is a need for a more mechanistical approach - trying to understand and investigate AVF maturation from a more cause-and-effect point of view, rather than an association point of view. In a way the complexity of any research approach should match the complexity of the clinical challenge. Currently, such new and innovative approaches build on the foundations of previous research, and advances in computer sciences and engineering now enable the wide application of patientspecific computational modeling.

The Hemodialysis Fistula Maturation (HFM) study is an example of a mechanistical approach. ${ }^{39}$ Although it is still a prospective observational (epidemiological) study, it aims to increase the understanding of AVF maturation rather ambitiously from four domains: anatomy (e.g. preoperative vessel size), biology (e.g. cellular functioning), clinic (e.g. patient comorbidity), and organization (e.g. surgical data). In the HFM study, they for example investigated how preexisting $\mathrm{NIH}$ can influence fistula maturation (only modestly despite its high prevalence) ${ }^{40,}{ }^{41}$ and how biomechanical properties (e.g. arterial stiffness assessed by pulse wave velocity measurement) are related to maturation. ${ }^{42,43}$ These are just two examples from two different domains they set out to investigate. The major strength of this epidemiological study is its size (i.e. approximately 600 patients), and its thoroughness in assessing many factors in four domains.

Unfortunately, the advances in patient-specific computational modeling are still limitedly exploited, and hemodynamical factors not incorporated in studies. Computational fluid dynamics (CFD) for example can be used to patient-specifically calculate WSSs ${ }^{44}$ - a metric that cannot be measured (yet). PWPMs can be used to predict postoperative flow. CFD and PWPM studies in the field of AVF research are still rather in their infancy. CFD studies usually include only a few patients, are not longitudinally designed, lack clinical outcomes, and are not always as patient-specific as they could be. The clinical application of a PWPM is part of this thesis and described in Chapter 6.

\section{PREOPERATIVE DUPLEX ULTRASONOGRAPHY}

Chapter 4 focused on the agreement of DUS measurements within and between observers (i.e. repeatability respectively reproducibility, together considered as 
precision). Although clinical guidelines recommend DUS assessment of arterial and venous diameters prior to any AVF creation, ${ }^{45-47}$ little is known about the intra- and interobserver agreement of the performed measurements. Insight in the variability of DUS is important for multiple reasons. Firstly, it helps with the interpretation of the measurements in clinical decision-making, and results from other studies reported in literature. Secondly, it can provide arguments for the ongoing debate regarding the routine use of DUS vessel mapping. Thirdly, it provides information for computational model performance and/or improvement.

The degrees of intra- and interobserver agreement on DUS measurements were found to be at least excellent and, hence, DUS examination was considered a reliable imaging modality. Two experienced vascular technicians assessed ten (pre)hemodialysis patients, and performed six representative measurements in triplicate (i.e. a venous diameter and arterial diameter and flow measurement in both the fore- and upper arm). The intraclass correlation coefficient (ICC) was used as a measure of agreement within and between the two vascular technicians.

Intraobserver agreement was classified as excellent (i.e. ICC 0.90 [0.74-0.97 95\% Cl] for the radial artery diameter), and interobserver agreement was also excellent (i.e. ICC 0.83 [0.46-0.96] for the upper arm cephalic vein diameter). As in clinical practice, exclusively the first of three measurements was used to determine the interobserver agreement. With the use of all three, the ICCs improved considerably.

\section{Interpreting diameter measurements in clinic and research}

Although preoperative diameter measurements overall show excellent intra- and interobserver agreement, it is important to realize that there are still minor differences when the individual patient is assessed. Consequently, we concluded that DUS in general is a reliable imaging modality, but guideline cut-off values should not be treated as absolute truths considering the uncertainty of these measurements.

Arterial and venous diameters are important factors for AVF maturation. ${ }^{16,48,49}$ Although the reported minimal diameter requirements for AVF maturation vary from $1.5^{48}$ to $3 \mathrm{~mm}^{50}$, increased vessel diameters are consistently related with increased maturation rates. Guidelines pragmatically recommend at least 2 or $3 \mathrm{~mm}$ arteries and veins for forearm or upper arm AVF creation respectively.

Recently, Wilmink and Corte-Real Houlihan challenged the diameter criteria. In their study on 803 AVFs, diameter measurement categories (i.e. <2.0, 2.0-2.1, 2.2-2.4, and $\geq 2.5 \mathrm{~mm}$ for forearm, and $<3.0,3.0-3.4,3.5-3.9$, and $\geq 4 \mathrm{~mm}$ for upper arm AVFs) showed no effect on functional dialysis use..$^{51}$ Given the history on how the 2 and $3 \mathrm{~mm}$ cut-off 
values were incorporated in guidelines, it comes to no surprise that the criteria are relative.

However, together with the extreme risk of bias in Wilmink and Corte-Real Houlihan's study, it is improper to conclude that diameter categories per se have limited value. Local protocol requirements actually included minimal cut-off values of 2 and $3 \mathrm{~mm}$ for forearm and upper arm AVFs respectively. Given the retrospective nature of the study, these values were ignored for good reasons in only approximately 5 percent of the cases. It is unclear if and how patients in whom the diameter requirements were ignored, differed in other terms from patients in whom the requirements were met. Patients with smaller caliber vessels for example could for example be overall healthier, younger, non-diabetic, etc.

\section{Routine preoperative duplex ultrasonography for arteriovenous fistula creation}

The findings of Chapter 4 do not provide a definitive answer in the ongoing debate regarding the usefulness of routine DUS. Recently, three systematic reviews with metaanalysis came to conflicting conclusions regarding routinely performing DUS examination in surgical work-up, while they were based on practically the same data.52-54 The vessel cut-off values differed considerably between the studies included in the systematic reviews. These differences, together with other methodological difficulties, rather than the appropriateness of DUS as an imaging modality, explain the ongoing debate.

The studies included in the reviews are methodologically flawed, and have an unclear or high risk of bias (assessed by the Cochrane risk of bias tool). ${ }^{53}$ Most strikingly, the randomized study by Nursal et al. included only patients after an AVF was already considered feasible based on physical examination (PE). ${ }^{55}$ To no surprise they found no differences in terms of FTM and patency rates between the PE and mapping group. Other studies did randomize on forehand, but allowed DUS to be used in the nonroutine group when PE was inconclusive. ${ }^{50,56}$ Although this is in effect routine vs. nonroutine, it does hamper fair comparison between studies. Furthermore, PE is in principle subjective (and researchers who publish on the matter presumably have ample experience), which in itself already limits the generalizability of any findings. 


\section{Implications for model performance}

Duplex ultrasonography examination is precise enough to serve as the imaging modality for model input parameter acquisition. Model output accuracy depends on the quality of the input data, which means that measurements need to be repeatable and reproducible.

Additional analyses of the data of Chapter 4, show that model performance can potentially be improved. The model simulates each AVF configuration 400 times, and each simulation uses a different set of patient-specific parameters to ensure that the parameter input space is completely covered, therewith evaluating the uncertainty of the flow predictions..$^{32}$ Each set consists of the measured input parameters \pm a prescribed uncertainty on specific parameters. This prescribed uncertainty, also called the uncertainty domain, acknowledges uncertainties in model input acquisition.

The uncertainty domain for arterial and venous diameter measurements is set to $\pm 10 \%$. For example, the parameter for a proximal forearm cephalic vein, which had a measured diameter of $2 \mathrm{~mm}$, is simulated with a random value between $1.8-2.2 \mathrm{~mm}$ in each of the 400 simulations. The uncertainty domain of $10 \%$ was based on available literature on the trueness of diameter measurements using ultrasound..$^{57}$ However, it does not include random errors introduced by observers.

When one wishes to take random errors by the observers into account, the uncertainty domains for arterial and venous measurements should, according to the results of Chapter 4, be $\pm 5-10$ respectively $\pm 10-20 \%$. These uncertainty domains are calculated by averaging the six diameter measurements per patient (three measurements by two observers), then calculating relative difference between the six individual measurement and the average, and then determining the minimum and maximum value of these relative differences. Obviously, this is a first order approach, and to fully appraise all uncertainties, more elaborate approaches are indicated.

It remains to be determined if model accuracy improves when these adjusted uncertainty domains are applied. Although in theory a more precise estimate of uncertainties would result in more accurate predictions, the opposite might also occur. Prescribing larger uncertainties can also result in unphysiological sets of input data, resulting in nonconvergent simulations (i.e. the equations cannot be solved anymore).

\section{Unassessed error sources - trueness or bias}

Measurement accuracy depends on the precision (i.e. random errors; the repeatability and reproducibility described above) and trueness (i.e. systematic errors). Although DUS 
examination is standardized in such a way that it is repeatable and reproducible, it might be incorrectly standardized, and hemodialysis patients per se are not standardized at all. Venous diameter measurement variance was found to mainly depend on the patient rather than congestion type (cuff vs. tourniquet), observer (two vascular technicians), and visit (patients were assessed on two separate days)..$^{58}$ Hemodynamic changes due to interdialytic changes in blood pressure ${ }^{59}$ and weight gain due to volume overload ${ }^{60}$ might also influence preoperative DUS examination results. In addition, the body composition of dialysis patients shows seasonal variability. Extracellular water and fluid overload were for example lower, and systolic blood pressure higher, in winter when compared with summer. ${ }^{61}$ This seasonal variability might in turn potentiate vessel dilatation in summer time due to higher ambient temperatures.

Other studies assessed various venous congestion methods to better appreciate venous diameters. Veins are highly compliant, and their diameters importantly depend on intravenous pressure. Proximal congestion then leads to a better appreciation of venous diameters, but there are numerous ways to dilate veins. Warm water submersion of the arm resulted in the largest venous diameters in comparison to the various other methods of hydrostatic, tourniquet, or cuff congestion. ${ }^{62,63}$ These studies, however, did not assess the effect of the various methods on AVF creation and maturation, nor did they assess the repeatability and reproducibility of diameter measurement under these methods. It remains therefore uncertain whether one should implement warm water submersion in scanning protocols.

The flow measurements, used to estimated peripheral resistance, are another source of error which is not assessed. The DUS scanning protocol required the sample volume to include the entire vessel lumen, and the time-averaged mean velocity was used to calculate the flow. This approach will always introduce a bias since low blood flow velocities near the wall are filtered - to remove wall movements. Alternatively, the timeaveraged peak velocity, within a (small) center-lumen sample volume can be used. This method is not affected by the wall filter, but now the velocity profile needs to be assumed (e.g. parabolic or flat).

\section{HEMODYNAMICAL CHANGES AFTER ARTERIOVENOUS FISTULA CREATION}

Chapter 5 deals with the natural hemodynamical changes after AVF creation, and importantly fills several important knowledge lacunae. It was found that AVFs require up to three months before vessel remodeling reached a plateau, in contrast to the prevailing notion of six weeks. Previous ipsilateral VAs, in addition, appear to cause a sort 
of vessel preconditioning that results in an accelerated maturation. The accelerated maturation, however, does not have clear beneficial or detrimental effects. Next to these new insights, the study also confirmed that the majority of the eventual AVF flow is reached almost instantaneously. ${ }^{64,65}$

These (new) findings are clinically relevant, but also guide future modeling research. Clinically, it provides a hemodynamical explanation for the clinical observation that functional maturation nowadays can take up to 13 weeks. ${ }^{22}$ The six week maturation period (as part of the Rule of $6 s$ for AVF maturation) was described in the National Kidney Foundation guidelines in 2006. The patient demographic has since then changed, which possibly explains the extended duration of maturation.

Chapter $\mathbf{5}$ only partially refutes the exclusion criterion in the multicenter randomized clinical trial of a previous ipsilateral VA. It was assumed that patients with a previous ipsilateral VA would show altered hemodynamics to such a degree that flow predictions would be inaccurate. Although preoperative vessel diameters and flows were indeed larger in patients with a previous ipsilateral VA, these values were also found in some of the individual patients without one. Though hemodynamics in patients with a previous ipsilateral VA have changed, this change was presumably not to such a degree that the model would have difficulties predicting immediate postoperative flows for these patients. Unfortunately, there were no direct postoperative flow measurements available of patients with a previous ipsilateral VA in the dataset to actually compare flow measurements and predictions.

The extended model by Manini et al. also predicts flow at six weeks postoperatively, ${ }^{66}$ but the accuracy of these predictions is most certainly negatively influenced by a previous ipsilateral VA. The extended model describes the change in vessel diameter in response to a deviation of peak WSS from a reference value. WSS values, as described above, do not entirely normalize after AVF creation. ${ }^{37}$ Consequently reference value might not be correct. More importantly, the mathematical algorithm also incorporates a numerical constant. This numerical constant is fitted on data on postoperative hemodynamical changes of patients without a previous ipsilateral VA. We have shown that the maturation process is different between the patients with and without a previous ipsilateral VA, and therefore the flow predictions at six weeks cannot be used.

\section{Improving maturation rates using patient-specific flow predictions}

The main objective of this thesis was to improve maturation rates by recommending a specific AVF configuration, based on patient-specific immediate postoperative flow predictions as additional preoperative information. Two chapters of this thesis were dedicated to this objective. 


\section{Trial design}

In Chapter $\mathbf{3}$ we presented the design of the multicenter randomized trial, which was designed in such a way that it would be truly representative for clinical practice resulting in liberal in- and exclusion criteria and limited standardization.

Basically the only exclusion criterion was a previous ipsilateral VA. RCTs often use elaborate in- and exclusion criteria that in turn might increase the likelihood of positive findings, but simultaneously limit generalizability.

Standardization was limited to the content and extent of the preoperative DUS examination (as this is mandatory for accurate simulations) and the 6 weeks follow-up visit (for primary endpoint assessment). All other procedures, interventions et cetera were left at the discretion of the treating physician. In this way, the effect of the clinical application of the computational model on maturation rates can properly be assessed, while the study simultaneously represents what effect could be expected when it is used in daily clinical practice.

\section{Model performance and maturation rates}

Chapter 6 shows that the immediate postoperative flow prediction was predictive for AVF maturation, but that maturation rates itself did not improve in the simulation group. FTM rates were in both groups approximately $30 \%$, which is in agreement with rates reported in the literature (20-40\%). ${ }^{22-24}$ The computational model did outperform the REDUCE-FTM score; the often studied FTM risk prediction model. ${ }^{15}$ The two main explanations why flow predictions did not improve maturation rates are: there is more to maturation than flow alone, and the large agreement between model recommendations and initial surgical plans.

The average immediate postoperative flow was $920 \pm 464$ versus $480 \pm 270 \mathrm{~mL} / \mathrm{min}$ for matured and FTM fistulae respectively $(p<.001)$. Although immediate postoperative flow differed significantly, there is large overlap in postoperative flow for the groups. Although it is thus impossible to discriminate maturing and failing fistulae based on postoperative flow, it can be used to assess likelihoods. Recently, Farrington et al. for example found that postoperative flow increase with an OR of 1.33 (95\% Cl 1.22-1.45) per $100 \mathrm{~mL} / \mathrm{min}$ was positively associated with AVF maturation. ${ }^{67}$ We have found similar results with an OR of 1.49 (1.27-1.75), confirming the predictive value of postoperative flow for AVF maturation. Flow predictions were also found to be predictive for AVF maturation, but offer the benefit that the AVF does not have to be created. The OR for flow predictions is $1.15(1.06-1.26)$ per $100 \mathrm{~mL} / \mathrm{min}$. The OR for flow predictions is lower 
than that of flow measurements, which can (partly) be explained by the overestimation of postoperative flow in failing fistulae.

Overall the predicted flow was $881 \pm 447$ respectively $669 \pm 321 \mathrm{~mL} / \mathrm{min}(\mathrm{p}<0.01)$ for mature and FTM fistulae; indicating a discriminating power also for immediate postoperative flow predictions. Subanalysis for clinical outcome (i.e. mature vs. FTM), indicates that the model loses its power in FTM fistulae. Measured and predicted flow are comparable in maturing fistulae with respectively $925 \pm 473$ and $819 \pm 399 \mathrm{~mL} / \mathrm{min}$ $(p=0.067)$. Conversely, in FTM fistulae they differ with respectively $480 \pm 270$ measured and $653 \pm 339 \mathrm{~mL} / \mathrm{min}$ predicted $(p=0.004)$. The model thus overestimates the immediate postoperative flow in fistulae that eventually fail. This finding (indirectly) confirms the notion that FTM is a consequence of an inadequate response to the altered hemodynamic state after AVF creation. On the other hand, model input can also explain this finding. Measurements might for example be more biased in patients that eventually fail. For example, the accuracy of flow measurements might be reduced in small arteries.

Several studies have shown a positive relationship between flow- and nitroglycerin mediated dilatation (FMD respectively NMD) and AVF maturation. ${ }^{43,68}$ FMD and NMD describe the ability of arteries to dilate in response to increased flow. FMD requires endothelial cells to signal vascular smooth muscle cell to relax, whereas in NMD nitroglycerin is used as a nitric oxide donor and is therefore endothelial cell independent. The flow increases tremendously after AVF creation, and consequently FMD is responsible for an adequate response to this new high flow state. FTM is possibly the result of an inadequate response to this flow increase by dysfunctional endothelial cells.

The second explanation for the absence of an effect on maturation rates is the high degree of agreement between recommendations and surgical plans. In $57 \%$ of the cases the postoperative flow prediction resulted in a recommendation that was in line with the initial surgical plan. This left $43 \%$ of the cases available to positively influence the surgical plan. Unfortunately, in $25 \%$ of the patients the physician did not act upon the recommendation, and in $6 \%$ there was no recommendation given.

Peroperative changes in the surgical plan, urgent dialysis indication, and inconsistency of physical examination with DUS findings, explain the majority of cases in which the recommendation was ignored, and are not related to model performance. Conversely, the inaccurate representation of the anatomy for simulation (i.e. $5 \%$ ) is related to model performance.

Peroperative change in surgical strategy can be due to fibrotic vessels as a result of venipunctures that become visible only during surgical exploration. Additionally, it has been suggested that plexus anesthesia can result in peroperatively changes of operative 
plans. ${ }^{69}$ An example of inconsistency between physical examination and DUS are veins that are visible on PE, but appear to be too small on DUS. In these cases, this would result in lower flow predictions but PE findings will prevail in surgical planning as this offers a more distal configuration.

There were seven instances in which no configuration was recommended. In only one this was due to nonconverging simulations. In the other cases the predictions for the three configurations were below and/or above the predefined cut-off values, which were based on literature. Especially the maturation cut-off (i.e. $\geq 400 \mathrm{~mL} / \mathrm{min}$ ) might not be accurate. Although this value should ensure sufficient flow at 6 weeks, the average predicted postoperative flow in FTM fistulae was considerably higher (i.e. $669 \mathrm{~mL} / \mathrm{min}$ ). The cut-off values would however only pose a difficulty when the flow predictions would continue to be dichotomized (i.e. insufficient or sufficient).

It is encouraging that the simulations are predictive for maturation, despite that the predictive power (for now) is too low to actually improve maturation rates. This is the first application of a highly innovative patient-specific modeling approach, and in comparison to conventional approaches it has some important advantages. Firstly, model performance is based on physics, and therefore not geographically or demographically influenced, whereas the currently available risk prediction models are. Secondly, we identified potential model improvements in this thesis, which would result in better model performance.

In addition, the computational model outperforms the well-known risk prediction score for FTM. The REDUCE-FTM score incorporates (only) four patient characteristics to predict the likelihood of AVF maturation. Although the C-statistic (a measure of goodness of fit) was promising in the initial study with 0.74 , this model has failed external validation to be of true value. When applied to other populations, the C-statistic dropped to 0.52 and 0.53 - indicating the model being slightly better than chance. ${ }^{23,70}$ Also in our study the REDUCE-FTM score was of no value with an AUC (area under the receiver operator characteristic curve; directly comparable with the C-statistic) of 0.53 . Conversely, the computational model had an AUC of 0.68 .

Focusing on the single factor of postoperative flow prediction to predict AVF maturation might appear inconsistent after the plea in Chapter $\mathbf{2}$ to do otherwise, but in this case it is not. Firstly, patient-specific flow predictions are a mechanistical approach. Postoperative flow is one of the few factors that have been consistently found to relate to AVF maturation. Having this factor available before surgery is then potentially very helpful. Secondly, the physical nature of the model is an important advantage - as discussed above. And, thirdly, it had first to be investigated whether this approach would actually relate to clinical outcomes before it could be implemented more broadly. 
Presumably, the model should in future applications be used in hybrid modeling approaches (e.g. part of prediction scores, node in machine learning algorithms). Although the model can predict AVF maturation, at the moment it does so insufficiently to be used separately. We have identified several potential model improvements. Chapter $\mathbf{4}$ indicates that the model's uncertainty domains can be improved, Chapter $\mathbf{5}$ indicates that the model might also be useful in patients with a previous ipsilateral VA, and based on Chapter 6 we think that adding an FMD metric can improve flow predictions for fistulae that eventually fail. The latter, would require future work to assess the underlying mechanisms FMD to incorporate it in governing equation of the model. Until then, it is also possible to use it empirically (e.g. multiplying the flow prediction with a certain FMD factor).

\section{TREATMENT STRATEGIES FOR FAILURE TO MATURE}

Despite all efforts FTM still remains a challenge in VA surgery, and Chapter $\mathbf{7}$ therefore reviews the available surgical and endovascular interventions to enhance maturation or to increase the number of functional fistulae. This review was on purpose not designed nor intended as a systematic review with meta-analysis. Although this consideration might be at the expense of methodological rigor, it benefits from the more thorough and complete overview of practically all of the available interventions.

The chapter has demonstrated that there is a multitude of interventions available, and it therewith confirmed the senselessness to group these into for example only surgical and/or endovascular interventions. The main finding is that similar results from endovascular and surgical approaches are reported in the literature. Consequently, it is plausible to leave the decision for any of these interventions at the discretion of the treating physician. There is obviously a strong need for well-designed and adequately powered randomized clinical trials to further determine the preferred treatment strategy.

Anastomotic adaptations are the most interesting ones in light of this thesis. The pSLOT (piggyback Straight Line Onlay Technique) ${ }^{12}$ and RADAR (Radial Artery Deviation And Reimplantation $)^{11}$ are examples of anastomotic adaptations. The authors of both techniques assume that their approach has favorable surgical techniques, which in addition result in favorable hemodynamical flow patterns postoperatively. Indeed, the initial results show reduced juxta-anastomotic stenosis development in both, but others have not yet adapted the new techniques.

The pSLOT technique is basically a side-to-side arteriovenous anastomosis in which the dissected cephalic vein "piggybacks" on the radial artery. The premise for this technique 
was that it would have a beneficial "torsional stress zone" in the first few venous centimeters. The conventional vein end-to-side artery anastomosis requires the vein to be mobilized in a three dimensional plane to the artery. This mobilization would then result in (micro)trauma to vein, which is then exposed to flow disturbances causing NIH. The reduced movement, and altered anastomosis angle would prevent this $\mathrm{NIH}$ development.

RADAR is an end-of-artery-to-side-of-vein arteriovenous anastomosis, and its premise was that the reduced surgical trauma to the vein results in fewer juxta-anastomotic stenoses. Presumably the extended no touch techniques play a major part in this technique. Only the internal aspect of the cephalic vein was dissected for $1.5 \mathrm{~cm}$, and by exsanguinating the arm under proximal cuff congestion at $250 \mathrm{mmHg}$ there was no need for clamping the vein or artery. The transected radial artery is then mobilized to the vein.

The claims of favorable hemodynamical patterns in both techniques could be examined by CFD. Although this has not been investigated, it might be that PSLOT indeed has a more favorable WSS distribution. The PSLOT technique effectively has a very steep anastomosis angle (i.e. $<5^{\circ}$ ). In a CFD study it has been shown that steeper angles (i.e. 30 and $45^{\circ}$ ) result in more favorable flow patterns in comparison to more shallow angels (i.e. 60 and $\left.90^{\circ}\right)^{71}$ This study, however, used highly idealized end-to-side geometries that might not correspond well with the PSLOT technique, and the most relevant WSS metric on which to base claims of beneficial patterns has still to be elucidated. ${ }^{72}$ Interestingly, a clinical study found the opposite ${ }^{73}$ in which an increased reintervention rate was observed in RCAVFs with anastomosis angles $<30^{\circ}$ - highlighting the importance of a collaborative approach by clinicians and engineers.

\section{CONCLUDING REMARKS AND FUTURE PERSPECTIVES}

This thesis has added to the understanding of AVF maturation in various ways, and tried to improve maturation rates by implementing a patients-specific flow prediction model in surgical planning.

There are several patient-specific modeling strategies available that show much potential, and already contributed significantly to the understanding of AVF maturation and functioning. Unfortunately, to date, computational models are often not as patientspecific or representative for clinical reality as they could be - or actually should be. To reap the benefits of patient-specific modeling, an increased interest for desk-to-bedside research is necessary. There is a need for larger studies that combine clinical and 
modeling approaches, use patient-specific segmentations, and importantly are longitudinally designed to allow model simulations on multiple time points.

Duplex ultrasonography was shown to be a reliable imaging modality in the preoperative work-up, despite its operator dependency. To settle the ongoing debate regarding routine use, or the value of diameter criteria, consensus should be reached regarding scanning protocols, and consequently well-designed (randomized) trial performed.

Arteriovenous fistula maturation might take longer than the prevailing notion of 6 weeks, as it has been shown that hemodynamical changes reach a plateau somewhere between 6 weeks and 3 months postoperatively. Although previous ipsilateral accesses appear to cause vessel preconditioning, there are no clear beneficial or detrimental effects thereof.

Patient-specific AVF simulations are found to be predictive for AVF maturation, but do not result in improved maturation rates when available in surgical planning. This is importantly due to an overestimation of postoperative flows of fistulae that eventually fail. Opportunities for model improvements are adjusting uncertainty domains, and incorporating an acute hemodynamical response metric in the model to better predict failing fistulae.

\section{Future perspectives}

This thesis has confirmed the benefits and advantages of patient-specific modeling in arteriovenous fistula research. The two main modeling families - PWPM and CFD should therefore be implemented increasingly in clinical practice. Clinicians and engineers will therefore need to collaborate more intensively. A better understanding of each other's point of view and research methods is hence indispensable.

The patient-specific flow predicting model can be improved by adjusting the uncertainty domains, and more importantly by incorporating the acute hemodynamical response to the tremendous flow increase after AVF creation. This latter improvement will enable the model to more accurately predict postoperative flow in especially failing fistulae, and therewith increase its discriminative power.

But even after model improvements, postoperative flow does not paint the entire picture - there are more factors involved in AVF maturation. Therefore, the potential of the model presumably lies in hybrid modeling approaches. The postoperative flow prediction can be incorporated in for example risk prediction scores or be part of machine learning algorithms.

Machine learning can importantly improve AVF research. To date it is still unclear which patient factors are predictive for AVF maturation. In part this is due to the conventional 
research methods. Patient characteristics are investigated based on theoretical grounds. Almost all factors to date share that they are related to overall vascular health: diabetes, ischemic heart disease, peripheral arterial disease, et cetera. Machine learning algorithms are not explicitly programmed where to look, and can therefore identify other factors that at first glance might be unrelated or theoretically farfetched. In addition, this approach can integrate and interpret data in ways conventional methods cannot. $^{74,75}$

Simultaneously, other metrics for vascular health should be explored. Presumably, part of the difficulties with the conventional risk factors is that they oversimplify complex diseases to a dichotomous factor. To exaggerate: there is a clear distinction between a diabetic that only uses a small dose of metformin, and one who unreliably takes insulin but is reliably drinking three liters of cola each day - they are both classified a diabetic. Perhaps studies can be improved when metrics for chronic glycemic variability are used rather than scoring diabetic yes or no. Perhaps using a lipid spectrum is more useful than scoring yes or no for ischemic heart disease or peripheral arterial disease.

\section{IN CONCLUSION}

This thesis has contributed to the understanding of AVF maturation, and importantly confirmed the potential of patient-specific computational modeling to improve maturation rates. In addition, it identified several possible improvements to capitalize this potential. 


\section{REFERENCES}

1. Pisoni RL, Zepel L, Port FK, et al. Trends in US Vascular Access Use, Patient Preferences, and Related Practices: An Update From the US DOPPS Practice Monitor With International Comparisons. American journal of kidney diseases. 2015.

2. Ravani P, Palmer SC, Oliver MJ, et al. Associations between hemodialysis access type and clinical outcomes: a systematic review. Journal of the American Society of Nephrology. 2013;24(3):465-73.

3. Lynch JR, Mohan S, McClellan WM. Achieving the goal: results from the Fistula First Breakthrough Initiative. Current opinion in nephrology and hypertension. 2011;20(6):583-92.

4. Lee T. Fistula First Initiative: Historical Impact on Vascular Access Practice Patterns and Influence on Future Vascular Access Care. Cardiovascular engineering and technology. 2017;8(3):244-54.

5. Masengu A, Hanko J. Patient factors and haemodialysis arteriovenous fistula outcomes. The journal of vascular access. 2017;18(Suppl. 1):19-23.

6. Wish JB. Catheter last, fistula not-so-first. Journal of the American Society of Nephrology : JASN. 2015;26(1):5-7.

7. Quinn RR, Ravani P. Fistula-first and catheter-last: fading certainties and growing doubts. Nephrology, dialysis, transplantation. 2014;29(4):727-30.

8. Lee T, Allon M. Reassessing Recommendations for Choice of Vascular Access. Clinical journal of the American Society of Nephrology. 2017;12(6):865-7.

9. Woo K, Ulloa J, Allon M, et al. Establishing patient-specific criteria for selecting the optimal upper extremity vascular access procedure. Journal of vascular surgery. 2017;65(4):1089-103 e1.

10. Lok CE, Davidson I. Optimal choice of dialysis access for chronic kidney disease patients: developing a life plan for dialysis access. Seminars in nephrology. 2012;32(6):530-7.

11. Sadaghianloo N, Declemy S, Jean-Baptiste E, et al. Radial artery deviation and reimplantation inhibits venous juxta-anastomotic stenosis and increases primary patency of radial-cephalic fistulas for hemodialysis. Journal of vascular surgery. 2016;64(3):698-706 e1.

12. Bharat $A$, Jaenicke $M$, Shenoy S. A novel technique of vascular anastomosis to prevent juxta-anastomotic stenosis following arteriovenous fistula creation. Journal of vascular surgery. 2012;55(1):274-80.

13. Chemla E, Tavakoli A, Nikam M, et al. Arteriovenous fistula creation using the Optiflow vascular anastomotic connector: the OPEN (Optiflow PatEncy and MaturatioN) study. The journal of vascular access. 2014;15(1):38-44.

14. Chemla E, Velazquez CC, D'Abate $F$, et al. Arteriovenous fistula construction with the VasQ external support device: a pilot study. The journal of vascular access. 2016;17(3):243-8.

15. Lok CE, Allon $M$, Moist $L$, et al. Risk equation determining unsuccessful cannulation events and failure to maturation in arteriovenous fistulas (REDUCE FTM I). Journal of the American Society of Nephrology. 2006;17(11):3204-12. 
16. Wong V, Ward R, Taylor J, et al. Factors associated with early failure of arteriovenous fistulae for haemodialysis access. European journal of vascular and endovascular surgery. 1996;12(2):207-13.

17. Tordoir JH, Rooyens P, Dammers R, et al. Prospective evaluation of failure modes in autogenous radiocephalic wrist access for haemodialysis. Nephrology, dialysis, transplantation. 2003;18(2):378-83.

18. Robbin ML, Chamberlain NE, Lockhart ME, et al. Hemodialysis arteriovenous fistula maturity: US evaluation. Radiology. 2002;225(1):59-64.

19. Rothuizen TC, Wong C, Quax PH, et al. Arteriovenous access failure: more than just intimal hyperplasia? Nephrology, dialysis, transplantation. 2013;28(5):1085-92.

20. Roy-Chaudhury P, Sukhatme VP, Cheung AK. Hemodialysis vascular access dysfunction: a cellular and molecular viewpoint. Journal of the American Society of Nephrology. 2006;17(4):1112-27.

21. Hentschel DM. Determinants of Arteriovenous Fistula Maturation. Clinical journal of the American Society of Nephrology. 2018.

22. Wilmink T, Hollingworth L, Powers S, et al. Natural History of Common Autologous Arteriovenous Fistulae: Consequences for Planning of Dialysis Access. European Journal of Vascular and Endovascular Surgery. 2016;51(1):134-40.

23. Masengu A, Maxwell AP, Hanko JB. Investigating clinical predictors of arteriovenous fistula functional patency in a European cohort. Clinical kidney journal. 2016;9(1):142-7.

24. Huijbregts $\mathrm{HJ}$, Bots $\mathrm{ML}$, Wittens $\mathrm{CH}$, et al. Hemodialysis arteriovenous fistula patency revisited: results of a prospective, multicenter initiative. Clinical journal of the American Society of Nephrology. 2008;3(3):714-9.

25. Basile $C$, Lomonte $C$, Vernaglione $L$, et al. The relationship between the flow of arteriovenous fistula and cardiac output in haemodialysis patients. Nephrology, dialysis, transplantation. 2008;23(1):282-7.

26. Scheltinga MR, van Hoek F, Bruijninckx CMA. Time of onset in haemodialysis accessinduced distal ischaemia (HAIDI) is related to the access type. Nephrology Dialysis Transplantation. 2009;24(10):3198-204.

27. Dhingra RK, Young EW, Hulbert-Shearon TE, et al. Type of vascular access and mortality in U.S. hemodialysis patients. Kidney international. 2001;60(4):1443-51.

28. Huberts W, Bode AS, Kroon W, et al. A pulse wave propagation model to support decision-making in vascular access planning in the clinic. Medical engineering \& physics. 2012;34(2):233-48.

29. van de Vosse FN, Stergiopulos N. Pulse Wave Propagation in the Arterial Tree. Annual Review of Fluid Mechanics. 2011;43(1):467-99.

30. Huberts W, Heinen SGH, Zonnebeld N, et al. What is needed to make cardiovascular models suitable for clinical decision support? A viewpoint paper. Journal of Computational Science. 2018;24:68-84.

31. Caroli A, Manini S, Antiga L, et al. Validation of a patient-specific hemodynamic computational model for surgical planning of vascular access in hemodialysis patients. Kidney international. 2013;84(6):1237-45. 
32. Bode AS, Huberts W, Bosboom EM, et al. Patient-specific computational modeling of upper extremity arteriovenous fistula creation: its feasibility to support clinical decision-making. PloS one. 2012;7(4):e34491.

33. Bozzetto $M$, Rota $S$, Vigo V, et al. Clinical use of computational modeling for surgical planning of arteriovenous fistula for hemodialysis. BMC Med Inform Decis Mak. 2017;17(1):26.

34. Roy-Chaudhury P, Spergel LM, Besarab A, et al. Biology of arteriovenous fistula failure. Journal of nephrology. 2007;20(2):150-63.

35. Remuzzi A, Ene-lordache B. Novel paradigms for dialysis vascular access: upstream hemodynamics and vascular remodeling in dialysis access stenosis. Clinical journal of the American Society of Nephrology. 2013;8(12):2186-93.

36. Brahmbhatt $A$, Remuzzi $A$, Franzoni $M$, et al. The molecular mechanisms of hemodialysis vascular access failure. Kidney international. 2016;89(2):303-16.

37. Ene-lordache $B$, Mosconi $L$, Antiga $L$, et al. Radial artery remodeling in response to shear stress increase within arteriovenous fistula for hemodialysis access. Endothelium. 2003;10(2):95-102.

38. Remuzzi A, Bozzetto $M$, Brambilla $P$. Is shear stress the key factor for AVF maturation? The journal of vascular access. 2017;18(Suppl. 1):10-4.

39. Dember LM, Imrey PB, Beck GJ, et al. Objectives and design of the hemodialysis fistula maturation study. American journal of kidney diseases. 2014;63(1):104-12.

40. Cheung AK, Imrey PB, Alpers CE, et al. Intimal Hyperplasia, Stenosis, and Arteriovenous Fistula Maturation Failure in the Hemodialysis Fistula Maturation Study. Journal of the American Society of Nephrology. 2017;28(10):3005-13.

41. Alpers CE, Imrey PB, Hudkins KL, et al. Histopathology of Veins Obtained at Hemodialysis Arteriovenous Fistula Creation Surgery. Journal of the American Society of Nephrology. 2017;28(10):3076-88.

42. Dember LM, Imrey PB, Duess MA, et al. Vascular Function at Baseline in the Hemodialysis Fistula Maturation Study. J Am Heart Assoc. 2016;5(7).

43. Allon M, Greene T, Dember LM, et al. Association between Preoperative Vascular Function and Postoperative Arteriovenous Fistula Development. Journal of the American Society of Nephrology. 2016;27(12):3788-95.

44. Taylor CA, Figueroa CA. Patient-specific modeling of cardiovascular mechanics. Annu Rev Biomed Eng. 2009;11:109-34.

45. Schmidli J, Widmer MK, Basile C, et al. Vascular Access: 2018 Clinical Practice Guidelines of the European Society for Vascular Surgery (ESVS). European Journal of Vascular and Endovascular Surgery. 2018;55(6):757-818.

46. Sidawy AN, Spergel LM, Besarab A, et al. The Society for Vascular Surgery: clinical practice guidelines for the surgical placement and maintenance of arteriovenous hemodialysis access. Journal of vascular surgery. 2008;48(5 Suppl):2S-25S.

47. NVVH. Richtlijn Shuntchirurgie. 2010.

48. Malovrh M. Non-invasive evaluation of vessels by duplex sonography prior to construction of arteriovenous fistulas for haemodialysis. Nephrology, dialysis, transplantation. 1998;13(1):125-9.

49. Malovrh M. Postoperative assessment of vascular access. The journal of vascular access. 2014;15 Suppl 7:S10-4. 
50. Ferring $M$, Claridge $M$, Smith $S A$, et al. Routine preoperative vascular ultrasound improves patency and use of arteriovenous fistulas for hemodialysis: a randomized trial. Clinical journal of the American Society of Nephrology. 2010;5(12):2236-44.

51. Wilmink T, Corte-Real Houlihan M. Diameter Criteria Have Limited Value for Prediction of Functional Dialysis Use of Arteriovenous Fistulas. European journal of vascular and endovascular surgery. 2018.

52. Wong CS, McNicholas N, Healy D, et al. A systematic review of preoperative duplex ultrasonography and arteriovenous fistula formation. Journal of vascular surgery. 2013;57(4):1129-33.

53. Kosa SD, Al-Jaishi AA, Moist L, et al. Preoperative vascular access evaluation for haemodialysis patients. The Cochrane database of systematic reviews. 2015;9:Cd007013.

54. Georgiadis GS, Charalampidis DG, Argyriou C, et al. The Necessity for Routine Preoperative Ultrasound Mapping Before Arteriovenous Fistula Creation: A Metaanalysis. European journal of vascular and endovascular surgery. 2015;49(5):600-5.

55. Nursal TZ, Oguzkurt L, Tercan F, et al. Is routine preoperative ultrasonographic mapping for arteriovenous fistula creation necessary in patients with favorable physical examination findings? Results of a randomized controlled trial. World journal of surgery. 2006;30(6):1100-7.

56. Smith GE, Barnes R, Chetter IC. Randomized clinical trial of selective versus routine preoperative duplex ultrasound imaging before arteriovenous fistula surgery. The British journal of surgery. 2014;101(5):469-74.

57. Huberts W, de Jonge $C$, van der Linden WP, et al. A sensitivity analysis of a personalized pulse wave propagation model for arteriovenous fistula surgery. Part A: Identification of most influential model parameters. Medical engineering \& physics. 2013;35(6):810-26.

58. Planken $\mathrm{RN}$, Keuter $\mathrm{XH}$, Hoeks $\mathrm{AP}$, et al. Diameter measurements of the forearm cephalic vein prior to vascular access creation in end-stage renal disease patients: graduated pressure cuff versus tourniquet vessel dilatation. Nephrology, dialysis, transplantation. 2006;21(3):802-6.

59. Santos SFF, Mendes RB, Santos CA, et al. Profile of Interdialytic Blood Pressure in Hemodialysis Patients. American journal of nephrology. 2003;23(2):96-105.

60. Abuelo JG. Large Interdialytic Weight Gains: Causes, Consequences, and Corrective Measures. Seminars in dialysis. 1998;11(1):25-32.

61. Broers NJ, Usvyat LA, Marcelli D, et al. Season affects body composition and estimation of fluid overload in haemodialysis patients: variations in body composition; a survey from the European MONDO database. Nephrology, dialysis, transplantation. 2015;30(4):676-81.

62. van Bemmelen PS, Kelly P, Blebea J. Improvement in the visualization of superficial arm veins being evaluated for access and bypass. Journal of vascular surgery. 2005;42(5):957-62.

63. Korten E, Spronk S, Hoedt MT, et al. Distensibility of forearm veins in haemodialysis patients on duplex ultrasound testing using three provocation methods. European journal of vascular and endovascular surgery. 2009;38(3):375-80. 
64. Shemesh D, Goldin I, Berelowitz D, et al. Blood flow volume changes in the maturing arteriovenous access for hemodialysis. Ultrasound in medicine $\&$ biology. 2007;33(5):727-33.

65. Robbin ML, Greene T, Cheung AK, et al. Arteriovenous Fistula Development in the First 6 Weeks after Creation. Radiology. 2016;279(2):620-9.

66. Manini S, Passera K, Huberts W, et al. Computational model for simulation of vascular adaptation following vascular access surgery in haemodialysis patients. Computer methods in biomechanics and biomedical engineering. 2014;17(12):1358-67.

67. Farrington $C A$, Robbin $M L$, Lee $T$, et al. Postoperative Ultrasound, Unassisted Maturation, and Subsequent Primary Patency of Arteriovenous Fistulas. Clinical journal of the American Society of Nephrology. 2018.

68. Malovrh M. Native arteriovenous fistula: preoperative evaluation. American journal of kidney diseases. 2002;39(6):1218-25.

69. Renaud CJ, Leong CR, Bin HW, et al. Effect of brachial plexus block-driven vascular access planning on primary distal arteriovenous fistula recruitment and outcomes. Journal of vascular surgery. 2015;62(5):1266-72.

70. Lilly MP, Lynch JR, Wish JB, et al. Prevalence of arteriovenous fistulas in incident hemodialysis patients: correlation with patient factors that may be associated with maturation failure. American journal of kidney diseases. 2012;59(4):541-9.

71. Ene-lordache B, Cattaneo L, Dubini G, et al. Effect of anastomosis angle on the localization of disturbed flow in 'side-to-end' fistulae for haemodialysis access. Nephrology, dialysis, transplantation. 2013;28(4):997-1005.

72. Browne LD, Bashar K, Griffin P, et al. The Role of Shear Stress in Arteriovenous Fistula Maturation and Failure: A Systematic Review. PloS one. 2015;10(12):e0145795.

73. Sadaghianloo N, Jean-Baptiste $E$, Rajhi K, et al. Increased reintervention in radialcephalic arteriovenous fistulas with anastomotic angles of less than 30 degrees. Journal of vascular surgery. 2015;62(6):1583-9.

74. Shameer K, Johnson KW, Glicksberg BS, et al. Machine learning in cardiovascular medicine: are we there yet? Heart. 2018;104(14):1156-64.

75. Deo RC. Machine Learning in Medicine. Circulation. 2015;132(20):1920-30. 
APPENDICES

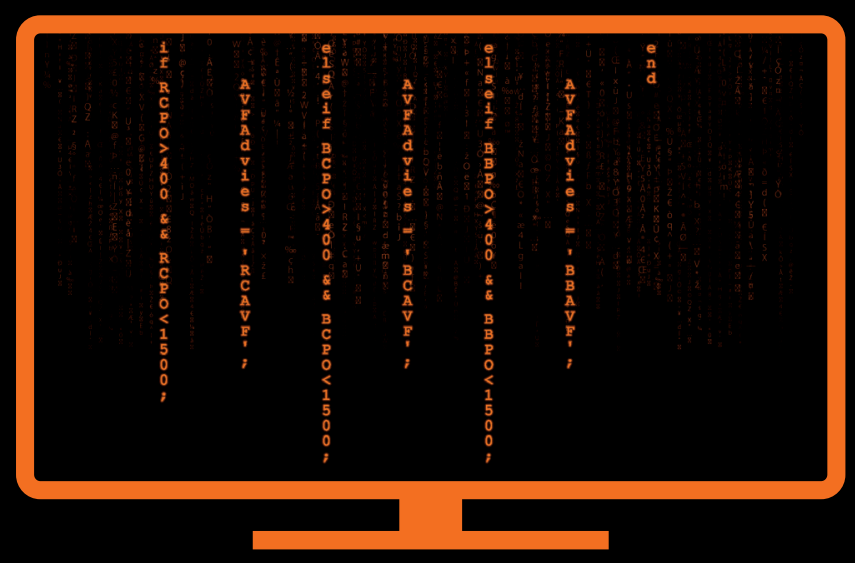



192 | APPENDIX 1 
Binnen de shuntchirurgie is het creëren van een natieve vaattoegang die gaat matureren en daardoor te gebruiken is voor hemodialyse waarschijnlijk de grootste uitdaging. Nonmaturatie of 'Failure to Mature' (FTM) is nog altijd één van de grootste struikelblokken en vormt het onderwerp van dit proefschrift.

De arterioveneuze fistel (AVF) of kortweg shunt, is de voornaamste vaattoegang gezien het gunstige profiel van voor- en nadelen in vergelijking met de alternatieve vaattoegangen; te weten de arterioveneuze graft (AVG) of de centraal veneuze katheter. Desalniettemin is nonmaturatie een groot probleem bij een shunt, ten dele doordat het proces van maturatie niet volledig doorgrond is.

Dit proefschrift poogt bij te dragen aan het begrip van shunt maturatie, waarbij het internationale streven naar de "right access for the right patient" een belangrijke bevestiging is van de noodzaak van dergelijk onderzoek. Het zwaartepunt van dit proefschrift ligt bij het toetsen of een eerder ontwikkeld computermodel in staat is de uitkomst van de klinische besluitvorming rondom shuntchirurgie positief te beïnvloeden. In vergelijking met eerdere pogingen om de uitkomsten van shuntchirurgie te verbeteren, heeft dit computermodel als voordeel dat het a) gebaseerd is op fysica, b) gepersonaliseerd kan worden voor de individuele patiënt en c) de uitkomstmaat postoperatieve bloedstroom is - een factor waarvan vaststaat dat deze sterk samenhangt met shuntmaturatie.

\section{ARTERIOVENEUZE FISTELS (SHUNTS) VOOR HEMODIALYSE}

In hoofdstuk 1 zijn de belangrijkste thema's van het proefschrift geïntroduceerd: de arterioveneuze fistel en het patiënt-specifieke computermodel. Wanneer patiënten het laatste stadium van nierfalen bereiken worden ze afhankelijk van nierfunctievervangende therapie, waarvan hemodialyse nog steeds één van de belangrijkste is. Een belangrijke voorwaarde is dan een goed functionerende vaattoegang zoals de shunt.

Een shunt wordt gecreëerd door een oppervlakkige ader (vene) en slagader (arterie) aan elkaar vast te hechten, waarbij een zogenaamde anastomose gemaakt wordt. Het bloed kan nu zonder noemenswaardige weerstand te ondervinden van slagader naar ader stromen. Hierdoor neemt de bloedstroom enorm toe, hetgeen een remodelleringsproces (maturatie) in gang zet. Tijdens maturatie treden er in principe drie veranderingen op in een periode van weken tot maanden. De bloedstroom neemt toe ten gevolge van een lagere weerstand, de veneuze diameter neemt toe door hogere wrijving van het bloed langs de vaatwand en de veneuze vaatwand wordt dikker in reactie op de hogere druk. Naast weerstand, wrijving en druk zijn er nog vele andere 
intra- en intercellulaire processen betrokken die nog niet allemaal (volledig) opgehelderd zijn.

De anastomose wordt bij voorkeur op één van drie locaties gemaakt: de pols, de elleboog of de bovenarm. Bloedvaten in de onderarm hebben in vergelijking met de bloedvaten in de bovenarm, een kleinere diameter en dus een hogere weerstand. Shunts in de onderarm hebben daarom een lagere bloedstroom en hogere kans op nonmaturatie (20-40\%). Shunts in de bovenarm hebben daarentegen een hogere bloedstroom en vormen daardoor een risico op het ontwikkelen van ischemische problemen in de onderarm (20\% van de gevallen).

Om met name het probleem van nonmaturatie het hoofd te bieden, is reeds een computermodel ontwikkeld dat in staat is voor de drie eerder genoemde shuntconfiguraties een patiënt-specifieke flowvoorspelling te doen. Dit computermodel is een zogenaamd Pulse Wave Propagation Model (PWPM), dat beschrijft hoe bloeddruken bloedstroomgolven in de vaatboom voortbewegen.

Om bovengenoemde beschrijving mogelijk te maken, wordt de relevante anatomie gesegmenteerd waarbij ieder segment de lokale relatie tussen druk en stroming (flow) beschrijft middels de natuurkundige parameters weerstand, compliantie en inertie. Deze beschrijving is gebaseerd op de natuurkundige wetten van massa- en momentumbehoud. Door middel van diametermetingen op de overeenkomstige anatomische locatie bij een patiënt kan dit model gepersonaliseerd worden.

\section{MODELLEERSTRATEGIËN IN SHUNTMATURATIE EN FUNCTIONEREN}

In hoofdstuk 2 wordt de complexiteit van shuntmaturatie in meer detail beschreven. Daarnaast worden de verschillende modelleerstrategieën, die van patiënt-specifieke computermodellen in het bijzonder, beschreven en op welke wijze deze bijdragend kunnen zijn. Het complexe samenspel van verschillende, afhankelijke factoren dat uiteindelijk leidt tot maturatie is tot op heden nog niet volledig duidelijk. Vast staat dat de vene tijdens maturatie naar buiten toe remodelleert en dat het functioneren van de shunt daarna afhangt van het niet optreden van neointimale hyperplasie (NIH). De balans tussen de gunstige remodellering en ongunstige $\mathrm{NIH}$ kan verstoord worden door meerdere factoren, die gebruikelijk worden onderverdeeld in up- en downstream events. Upstream events zijn die factoren die schade toebrengen aan endotheel- en gladde spiercellen, welke vervolgens de downstream events in beweging brengen. Downstream events resulteren uiteindelijk in NIH via een complex samenspel van cellen, cytokines en signaalcascades. 
Zowel in de up- als downstream events is een belangrijke rol weggelegd voor flow en wall shear stress (WSS). WSS is de wrijvingskracht die stromend bloed uitoefent op de vaatwand en wordt door endotheelcellen waargenomen door deformatie van het cytoskelet. Wanneer de flow voor een gegeven diameter toeneemt zal dit leiden tot een hogere WSS en dientengevolge een ernstigere deformatie van het cytoskelet. In reactie daarop wordt relaxatie van de gladde spiercellen in de vaatwand geïnitieerd, waardoor de diameter toeneemt en de WSS weer daalt tot binnen de fysiologische waarden.

$\mathrm{Na}$ het creëren van een shunt zal de flow enorm toenemen en de vaatwand dus blootgesteld worden aan onfysiologisch hoge WSS. In de weken tot maanden na aanleg zal echter weer een nieuw, bijna fysiologisch evenwicht bereikt worden, waarbij met name de piek WSS als belangrijkste determinant voor maturatie verondersteld wordt.

Ondanks het bekende belang van bovengenoemde hemodynamische factoren richten veel onderzoeken zich op patiëntkarakteristieken die een afgeleide zijn voor cardiovasculaire gezondheid: perifeer arterieel vaatlijden, cardio- en cerebrovasculaire aandoeningen, hypertensie, diabetes, et cetera. Hoewel sterk verschillend, hebben ze één eigenschap met elkaar gemeen: ze simplificeren complexe en multifactoriële aandoeningen en worden vervolgens (separaat) gebruikt om de uitkomst van een ander complex en multifactorieel proces zoals maturatie te voorspellen. Het is dan geenszins een verrassing dat maturatie onvolledig begrepen wordt.

Om die reden hebben wij gepleit voor een meer mechanistische aanpak van het probleem, waarbij de nadruk meer op causale in plaats van associatieve verbanden gelegd wordt. In zekere zin zou de complexiteit van de onderzoekingen in verhouding moeten zijn met die van het probleem dat onderzocht wordt. Belangrijke vorderingen in computer- en biomedische wetenschappen maken een dergelijke aanpak tegenwoordig mogelijk, in het bijzonder die van patiënt-specifieke computermodellen.

Helaas worden de mogelijkheden van patiënt-specifieke computermodellen nog niet ten volle benut, noch worden hemodynamische factoren veelvuldig meegenomen in epidemiologische of observationele studies. WSS, een maat die momenteel niet gemeten kan worden, kan bijvoorbeeld wel worden berekend voor een individu middels computational fluid dynamics (CFD). De beschikbare studies die CFD toepassen op klinische vraagstukken omtrent shunts beschrijven echter vaak slechts kleine patiëntaantallen, zijn niet longitudinaal van opzet of hebben geen klinisch relevante uitkomstmaat. Het eerder genoemde PWPM wordt later in detail behandeld, evenals de studieopzet van de ShuntSimulatieStudie (hoofdstuk 3). Deze zal dan in samenhang met de eerste resultaten (hoofdstuk 6) worden besproken. 


\section{PREOPERATIEVE DUPLEX ECHOGRAFIE}

Hoofdstuk 4 richt zich op de nauwkeurigheid van duplexmetingen. Ondanks dat klinische richtlijnen het meten van veneuze en arteriële diameters adviseren ter voorbereiding op het creëren van een shunt, is er weinig tot niets bekend wat betreft de intra- en interobserver overeenstemming van die metingen. Inzicht hierin is belangrijk om meerdere redenen: ten eerste ondersteunt het de interpretatie van deze metingen in besluitvorming en die van andere onderzoeken in de literatuur; ten tweede biedt het argumenten in het bestaande debat of de geadviseerde metingen routinematige verricht moeten worden; ten derde kan het sturing geven aan de interpretatie of verbetering van het functioneren van het gebruikte PWPM.

Uit het hoofdstuk blijkt dat de mate van intra- en interobserverovereenstemming minimaal excellent was en duplex dus als betrouwbare modaliteit gezien kan worden. Twee ervaren vaatlaboranten hebben tien (pre)dialyse patiënten onderzocht, waarbij zij zes representatieve metingen hebben verricht in drievoud: één veneuze diametermeting en één arteriële diameter- en flowmeting in zowel de onder- als bovenarm. De intraclass correlation coefficient (ICC) werd gebruikt om de mate van overeenstemming te kwantificeren, lopende van 0 (geen) tot 1 (perfecte overeenstemming). De laagste ICC voor de intraobserverovereenstemming was die voor de arteria radialis diameter $(0.90 ; 0.74-0.9795 \% \mathrm{Cl})$ en de laagste ICC voor interobserverovereenstemming was die voor de bovenarm vena cephalica diameter $(0.83 ; 0.46-0.96)$.

\section{HEMODYNAMISCHE VERANDERINGEN NA HET CREËREN VAN EEN SHUNT}

Hoofdstuk 5 omvat een studie naar de natuurlijke hemodynamische veranderingen die optreden nadat een shunt gecreëerd is. In deze studie werd gevonden dat een shunt tot drie maanden na aanleg nog hemodynamische veranderingen kan vertonen, in tegenstelling tot de vigerende regel dat de maturatietijd zes weken bedraagt. Daarnaast werd gevonden dat een eerdere vaattoegang aan dezelfde zijde leidt tot een zekere mate van preconditionering en dat in die gevallen het maturatieproces mogelijk sneller verloopt. Tegelijkertijd waren er geen duidelijke aanwijzingen dat deze preconditionering of versnelde maturatie leidt tot voor- of nadelen. Naast deze nieuwe inzichten, bevestigt dit onderzoek (nogmaals) dat het merendeel van de postoperatieve 
flowtoename vrijwel instantaan behaald wordt. Een observatie die vaker in de literatuur beschreven is.

De bevindingen uit dit hoofdstuk bieden een hemodynamische verklaring waarom functionele maturatie tegenwoordig tot 13 weken kan duren, in tegenstelling tot de eerder genoemde zes weken. Een mogelijke verklaring daarvoor is dat dit zes weken criterium opgesteld is in een richtlijn gepubliceerd in 2006, terwijl de patiëntdemografie sindsdien in belangrijke mate veranderd is.

\section{HET VERBETEREN VAN SHUNTMATURATIE MIDDELS PATIËNT- SPECIFIEKE FLOWVOORSPELLINGEN}

De nu volgende hoofdstukken (3 en 6) vormen de kern van dit proefschrift: de ShuntSimulatieStudie. Het doel van deze studie was shuntmaturatie te verbeteren door een bepaalde shuntconfiguratie aan te bevelen op basis van patiënt-specifieke flowvoorspellingen.

\section{Studieopzet}

In hoofdstuk 3 wordt de studieopzet beschreven van de ShuntSimulatieStudie. Het betreft een multicenter gerandomiseerde studie, die zo is opgezet dat het representatief is voor de klinische realiteit. Oftewel, er is een vrij liberale inclusie en minimale standaardisatie toegepast. Een eerdere vaattoegang was praktisch de enige reden waarom patiënten niet konden deelnemen. Er is bewust voor deze liberale inclusie gekozen omdat bij het toepassen van uitgebreide in- en exclusiecriteria de kans op positieve bevindingen mogelijk toeneemt terwijl de generaliseerbaarheid gelimiteerd wordt.

Standaardisatie was beperkt tot de inhoud en uitgebreidheid van het preoperatieve duplexonderzoek en het zes weken follow-up moment. Het gestandaardiseerde preoperatieve duplexonderzoek was noodzakelijk voor adequate inputdata voor de simulaties, terwijl de gestandaardiseerde inhoud van het zes weken follow-up moment noodzakelijk was om het primaire eindpunt vast te kunnen stellen. Alle besluiten rondom overige zaken en procedures, zoals wanneer te interveniëren bij het vermoeden op een stenose, zijn overgelaten aan de behandelend arts. Op deze wijze wordt zo min mogelijk ingebroken op de klinische praktijk en kan daadwerkelijk getoetst worden wat het effect van het computermodel op de klinische uitkomsten is. 


\section{Resultaten}

Hoofdstuk 6 beschrijft de eerste resultaten van de ShuntSimulatieStudie, waarvoor 236 patiënten gerandomiseerd zijn voor ófwel de controlegroep (116 patiënten) óf de simulatiegroep (120 patiënten). De studie toont aan dat de patiënt-specifieke flowvoorspellingen voorspellend zijn voor shuntmaturatie, ondanks dat de proportie gematureerde shunts niet toegenomen is in de simulatiegroep ten opzichte van de controlegroep. De gevonden proportie van ongeveer 30\% nonmaturatie (in beide groepen) is representatief voor de klinische realiteit.

De twee belangrijkste verklaringen voor deze bevindingen zijn: 1) dat er meer factoren van belang zijn dan flow alleen en 2 ) de grote mate van overeenstemming tussen advies en het initiële chirurgische plan. De voorspellende waarde van het computermodel presteert daarentegen wel beter dan het meest bestudeerde predictiemodel op dit moment, REDUCE-FTM, dat eveneens probeert te voorspellen welke shunt zal gaan matureren.

Om met de eerstgenoemde factor verder te gaan; de gemiddelde direct postoperatief gemeten flow was $920 \pm 464$ versus $480 \pm 270 \mathrm{~mL} / \mathrm{min}$ voor gematureerde en gefaalde shunts respectievelijk $(p<0.001)$. Hoewel er dus een statistisch significant verschil tussen beide is, vertonen beide groepen grote overlap. Het is dus onmogelijk alleen op grond van postoperatieve flow te voorspellen of een shunt zal matureren of falen, maar het kan wel helpen bij het inschatten van kansen. Recent heeft Farrington et al. aangetoond dat een $100 \mathrm{~mL} / \mathrm{min}$ flowtoename een odds ratio (OR) heeft van $1.33(1.22-1.4595 \% \mathrm{Cl})$ op het matureren van de shunt. Wij hebben een vergelijkbaar getal gevonden met een OR van 1.49 (1.27-1.75), waarmee de voorspellende waarde van postoperatieve flow bevestigd wordt.

Voor de direct postoperatieve flowvoorspelling geldt eveneens een positieve OR, zij het iets minder sterk met $1.15(1.06-1.26)$ per $100 \mathrm{~mL} / \mathrm{min}$ flowtoename. Dit getal heeft echter als belangrijk voordeel dat het bekend is zonder dat een shunt aangelegd hoeft te zijn.

De verklaring voor de gevonden discrepantie tussen meting en voorspelling moet hoogstwaarschijnlijk gezocht worden in de overschatting van de postoperatieve flow van shunts die uiteindelijk falen. De voorspelde flow was namelijk $881 \pm 447$ respectievelijk $669 \pm 321 \mathrm{~mL} / \mathrm{min}(\mathrm{p}<0.01)$ voor gematureerde en gefaalde shunts. Wanneer echter vanuit klinische uitkomst gekeken wordt naar gemeten en voorspelde flow, blijkt dat deze met respectievelijk $925 \pm 473$ en $819 \pm 399 \mathrm{~mL} / \mathrm{min}$ gelijk zijn voor gematureerde shunts $(p=0.067)$, terwijl dit wel statistisch significant verschillend was voor gefaalde shunts met respectievelijk $480 \pm 270$ en $653 \pm 339 \mathrm{~mL} / \mathrm{min}(p=0.004)$. Het model lijkt dus falende shunts te overschatten, hetgeen een indirect bewijs is voor de 
hypothese dat nonmaturatie een inadequate respons is op de veranderde hemodynamiek.

Er zijn onderzoeken beschikbaar die hebben gekeken naar hemodynamische adaptatiecapaciteit. Daarbij is een positieve relatie aangetoond tussen flow- en nitroglycerine-gemedieerde dilatatie (FMD respectievelijk NMD) en shuntmaturatie. Zowel FMD als NMD is een maat die aangeeft in welke mate een arterie kan dilateren in reactie op een toegenomen flow. Hierbij is FMD afhankelijk van een goede endotheelfunctie om de gladde spiercellen te laten ontspannen, terwijl NMD dit direct doet door toediening van nitroglycerine zonder tussenkomst van endotheelcellen. $\mathrm{Na}$ aanleg van een shunt neemt de flow aanzienlijk toe, waarbij middels FMD een balans gezocht wordt in deze nieuwe staat van hyperhemodynamiek. Bij falende shunts is deze reactie mogelijk inadequaat.

De tweede verklaring voor de afwezigheid van een effect van het computermodel op de klinische uitkomst, is de grote mate van overeenstemming tussen advies en het initiële chirurgische plan. In 57\% van de gevallen waren deze in overeenstemming, waardoor slechts $43 \%$ beschikbaar bleef om een effect op de klinische besluitvorming te bewerkstelligen. Helaas werd daarbij in 25 procentpunt geen gehoor gegeven aan het advies en werd in 6 procentpunt geen advies afgegeven.

In de gevallen waarbij het advies genegeerd werd, waren de voornaamste redenen het peroperatief veranderen van het plan (bijvoorbeeld vanwege fibrotische venen die pas zichtbaar werden bij chirurgische exploratie), spoedige dialyseafhankelijkheid waardoor het wachten op maturatie ongewenst is en inconsistentie tussen bevindingen bij lichamelijk onderzoek en duplex echografie.

In de zeven gevallen waarbij er geen advies afgegeven werd, was dit in slechts één geval doordat de simulaties niet convergeerden. In de andere gevallen waren de voorspellingen voor de verschillende configuraties alle boven of onder de op literatuur gebaseerde afkapwaarden. Met name de afkapwaarde voor maturatie is mogelijk niet accuraat, gezien de gemiddelde flowvoorspelling voor gefaalde shunts met $699 \mathrm{~mL} / \mathrm{min}$ aanzienlijk hoger was dan de vooraf gedefinieerde afkapwaarde van $400 \mathrm{~mL} / \mathrm{min}$. Indien de flowvoorspelling uiteindelijk gedichotomiseerd gebruikt wordt (voldoende/onvoldoende voor maturatie), zal de afkapwaarde opnieuw gedefinieerd moeten worden. De gevonden resultaten van de ShuntSimulatieStudie zijn bemoedigend voor vervolgonderzoek. Ondanks dat de voorspellende waarde (vooralsnog) te zwak is om op groepsniveau uitkomsten te verbeteren, bevestigt het wel de potentiële meerwaarde van patiënt-specifieke flowvoorspellingen. Bovendien is het de eerste klinische toepassing van deze hoogst innovatieve aanpak van het lang bestaande probleem van nonmaturatie, waarbij in dit proefschrift reeds een aantal mogelijke modelverbeteringen geïdentificeerd zijn. 
In vergelijking met de REDUCE-FTM score presteert het computermodel aanzienlijk beter. De REDUCE-FTM score voorspelt de waarschijnlijkheid van FTM op basis van vier patiëntkarakteristieken. Hoewel met deze score initieel een C-statistic (een maat voor goodness of fit) van 0.74 behaald werd, is deze C-statistic bij externe validatie in twee studies aanzienlijk lager met slechts 0.52 en 0.53 - net iets beter dan kans. In onze steekproef was de C-statistic eveneens 0.53 voor de REDUCE-FTM score, terwijl het patiënt-specifieke computermodel 0.68 wist te behalen.

Desalniettemin zal de toepassing van het computermodel in hybride modellen liggen, waarbij het een onderdeel zal zijn van een predictiescore of als node gebruikt zal worden in machine learning algoritmen. Het model kan weliswaar flow voorspellen, maar deze voorspelling is momenteel nog onvoldoende sterk om het model separaat toe te kunnen passen. De mogelijke verbeteringen van het model zijn bijvoorbeeld het aanpassen van onzekerheidsdomeinen op basis van de bevindingen in hoofdstuk 4 of de flowvoorspellingen voor met name falende fistels te verbeteren door een maat voor FMD te incorporeren.

\section{BEHANDELING VAN FAILURE TO MATURE}

Vermoedelijk zal FTM tot op zekere hoogte altijd een uitdaging blijven binnen de shuntchirurgie. Om die reden is in hoofdstuk 7 de literatuur inzake chirurgische en endovasculaire strategieën ter voorkoming of behandeling van FTM samengevat. Chirurgische interventies waren bijvoorbeeld alternatieve anastomoses (pSLOT, RADAR), anastomose implantaten (Optiflow) of een proximale neoanastomose. Endovasculaire interventies waren onder andere PTA van de veneuze afvloed of anastomose, balloon assisted maturation of het occluderen van accessoire venen.

De belangrijkste bevinding is misschien wel dat er bijzonder veel verschillende strategieën zijn, zowel chirurgisch als endovasculair, die vrijwel allemaal vergelijkbare resultaten laten zien. Derhalve is geconcludeerd dat de keuze voor een behandeling het beste overgelaten kan worden aan de behandelend arts, zodat deze bijvoorbeeld de lokale faciliteiten kan laten meewegen in de beslissing.

\section{CONCLUSIE EN TOEKOMSTPERSPECTIEVEN}

Het in dit proefschrift beschreven werk heeft op verschillende manieren bijgedragen aan het begrip van shuntmaturatie. Daarnaast heeft dit werk gepoogd de maturatiekans 
van een AVF gunstig te beïnvloeden door de klinische toepassing van een innovatief patiënt-specifiek flowvoorspellend computermodel.

Er zijn reeds verschillende patiënt-specifieke modelleerstrategieën beschikbaar die veel potentie laten zien, maar nog in onvoldoende mate worden toegepast en/of onvoldoende representatief zijn. Ten einde het potentieel van deze innovatieve strategieën te benutten zou er meer aandacht moeten zijn voor desk-to-bedside onderzoek. Er moeten grotere studies komen die epidemiologische modellen en computermodellen combineren. Deze studies dienen in het bijzonder longitudinaal te zijn om de patiënt-specifieke simulaties op meerdere tijdspunten te kunnen uitvoeren. Het matureren van een shunt heeft tegenwoordig, met een veranderde patiëntdemografie, mogelijk meer tijd nodig dan de huidig aangenomen tijd van zes weken. Het is aangetoond dat de hemodynamische veranderingen tussen de zes weken en drie maanden een plateau bereiken. En hoewel een eerdere ipsilaterale vaattoegang mogelijk tot preconditionering van de bloedvaten leidt, is daarvan geen duidelijk voorof nadeel te verwachten.

Patiënt-specifieke flowvoorspellingen zijn voorspellend voor shuntmaturatie, maar deze voorspelling is onvoldoende sterk om tot een gunstige beïnvloeding van klinische besluitvorming te leiden. Dit onvermogen wordt voornamelijk bepaald door een overschatting van de voorspelde postoperatieve flow van shunts die uiteindelijk falen. Er zijn een aantal mogelijke verbeteringen voor het model aangedragen zoals het aanpassen van de onzekerheidsdomeinen van het computermodel en het incorporeren van een maat van FMD.

\section{Toekomstperspectieven}

Dit proefschrift heeft de kansen en voordelen van patiënt-specifieke computermodellen bevestigd, waarbij geconcludeerd werd dat de twee belangrijkste modelfamilies (CFD en PWPM) meer toegepast kunnen en moeten worden in de klinische praktijk. Artsen en ingenieurs zouden daartoe meer moeten samenwerken, waarbij een wederzijds begrip van de verschillende onderzoeksstrategieën en -mogelijkheden van belang is.

Zelfs wanneer het patiënt-specifieke flowvoorspellende model verbeterd wordt met eerder aangedragen suggesties, zal postoperatieve flow niet het volledige verhaal van maturatie vertellen. Er zijn immers meer factoren die van invloed kunnen zijn. Daarom zal de toekomstige toepassing van een dergelijk model meer in hybride toepassingen gezocht moeten worden, bijvoorbeeld als onderdeel van machine learning algoritmen. Machine learning is een nog redelijk onontgonnen gebied binnen shuntchirurgisch onderzoek, maar kan in potentie belangrijke inzichten verschaffen. De invloed van eerder genoemde patiënt-karakteristieken is nog steeds niet onomstotelijk vastgesteld. 
Ten dele komt dit door de manier van conventioneel onderzoek; op theoretische gronden worden factoren geïdentificeerd die mogelijk van invloed kunnen zijn, waarna deze mogelijke associatie onderzocht wordt in veelal observationele studies. De factoren zijn daarnaast vrijwel allemaal een afgeleide voor de algehele gezondheid en in het bijzonder de gezondheid van de bloedvaten (diabetes, ischemische hartziekten, perifeer arterieel vaatlijden). Een belangrijk voordeel van machine learning algoritmen is dat niet expliciet geïnstrueerd wordt welke factoren meegenomen dienen te worden. Daarnaast kunnen dergelijke algoritmen gebruikt worden om data op andere wijze te integreren of te interpreteren dan met conventionele onderzoeksmethoden mogelijk is. 
Nederlandse Samenvatting | 203 

206 | APPENDIX 2 
Chronic kidney disease (CKD) remains an important cause of morbidity and mortality worldwide. Incidence and prevalence numbers increase or at least plateaued both nationally and internationally. Patients with advanced stages of CKD rely on renal replacement therapy, of which hemodialysis is the predominantly used modality.

A vascular access is required to facilitate hemodialysis - the artificial kidney needs access to the patient's bloodstream. The arteriovenous fistula (AVF) is the access of choice over arteriovenous grafts and central venous catheters despite its risk of failure to mature (FTM).

Failure to mature describes the unsuccessful adaptation of the native vessels to the altered hemodynamics after the creation of an arteriovenous anastomosis, and occurs in $20-40 \%$ of all surgically created AVFs for hemodialysis. Consequently, FTM is an important issue that receives clinical and scientific attention. This thesis reports on the results of probably one of the most innovative approaches to date to reduce FTM rates. In this chapter we will discuss the valorization opportunities that originate from the work presented in this thesis. Valorization can be subdivided into two aspects. First, knowledge can be me made available for economical and societal exploration. Second, this knowledge can be translated into new products, services, processes, and businesses.

\section{MAKING KNOWLEDGE AVAILABLE AND SUITABLE FOR ECONOMIC AND SOCIETAL EXPLORATION}

The society of today increasingly asks academia to perform relevant research that will benefit the society, either through direct implementation of the results or (indirectly) through communication of studies and results thereof to academic peers. Additionally, scientific training of medical PhD candidates results in physicians with improved skills to critically acclaim scientific studies - well needed trait in the era of evidence based medicine, with a worldwide, ever increasing scientific output.

Many of the chapters are or will be published in peer-reviewed journals, therewith distributing the acquired knowledge to the academic/clinical community. In addition, parts of this thesis and other related work is presented on (inter)national conferences.

\section{Direct valorization}

The first and foremost direct result was the implementation of a more standardized duplex ultrasonography scanning protocol in several Dutch hospitals. For participation in the Shunt Simulation Study a predefined and uniform duplex scanning protocol was necessary. Although clinical guidelines recommend duplex examination to be a routine 
part of AVF creation work up, surprisingly there was large variation in how this duplex examination was performed. This heterogeneity was observed for both pre- and postoperative examination. Measurement standardization (input) was necessary to guarantee accurate flow predictions (model output).

Protocol standardization proved to be well received in several centers where physicians were accustomed to summarily duplex protocols. Our more elaborated protocol was advantageous since it provided them with more information for surgical planning or a better indication of the possible anatomical substrate in case of AVF dysfunction. Consequently, in these centers the now standardized duplex protocol was retained, even after patient inclusion or follow-up visits were finished.

\section{Indirect valorization}

Other results of this thesis will be beneficial more indirectly. Chapter 2 for example aims to inform the relative lay reader with the pros and cons, and the necessary preconditions for computational modeling, it identifies the accomplishments to date, and it suggests improvements for further research. It was observed that patient-specific modeling is gaining momentum, also in AVF research. It therefore is also increasingly being published in clinical journals. Unfortunately, in many of the studies methodological difficulties are identified. Increasing the understanding of such studies will lead to a more critical appraisal of the methodology and/or results.

In Chapter 4 it was shown that duplex ultrasonography is a reliable imaging modality to support decision-making in AVF creation. Internationally there is an ongoing debate whether or not duplex scanning should be performed routinely during surgical planning. With the findings from this chapter, additional arguments are provided that the unequivocal results of previous studies are primarily due to methodological difficulties.

Chapter 5 adds to the (hemodynamical) understanding of arteriovenous fistula maturation. Currently, the Rule of Sixes for AVF maturation is questioned by the international scientific community in many peer-reviewed publications. Researchers and physicians increasingly recognize the relatively anecdotal evidence of the Rule of Sixes. The study described in this chapter was the first to investigate the postoperative hemodynamical changes for a relatively long period in a large cohort of patients. It therewith provides a better hemodynamical understanding of AVF maturation.

Chapter 6 most importantly confirms the potential benefit of adding patient-specific flow predictions to AVF surgery planning. Until now, it was only known that the patientspecific flow predictions were accurate, but if and to which extent they could influence clinical outcomes was still undetermined. In a way this adds to the valorization of the 
previous research that was performed in designing the computational model. Additionally, the findings of this chapter might also spark future interest for or confidence in patient-specific computational modeling.

TRANSLATING KNOWLEDGE INTO PRODUCTS, SERVICES, PROCESSES, AND NEW BUSINESSES

The most obvious possibility to translate the knowledge described in this thesis is to convert the computational model into (stand-alone) software or web-based application. Here, the physician can enter the relevant patient-specific data, and a flow prediction will be calculated or sent to the physician in a timely manner. Till the moment that desktop computers have the computational power of nowadays servers with parallel computing, the major potential to translate the knowledge is probably to provide an integral AVF surgical planning service. This service would then include training of vascular technicians, standardization of duplex examination, and training physicians in clinical decision-making, including how to interpret duplex measurements, which may be with or without the incorporation of patient-specific flow predictions. 

212 | APPENDIX 3 
Om met de woorden van de grote filosoof Ye West (voorheen Kanye West) te beginnen: "It's hard to be humble when you stuntin' on a Jumbotron". Het is natuurlijk moeilijk bescheiden te blijven (dixit Peter Blanker) met mijn naam op het grote scherm en de kaft, maar toch moet ik nederig zijn - aldus Kendrick Lamar. Hoewel ik het, net als Frank Sinatra, op mijn manier heb gedaan kan ik het, net als De Dijk, niet alleen. En daarom ga ik net als Earth, Wind \& Fire mijn dankbaarheid tonen. Oftewel, werk aan de winkel, zoals Opgezwolle zou zeggen. Niks gaat vanzelf.

Genoeg muzikale verwijzingen. Hier ligt mijn proefschrift en dit is het meest gelezen hoofdstuk.

Geachte dr. Tordoir, beste Jan, allereerst richt ik mij tot jou. Bedankt! Ik kan me geen betere promotor wensen. Inderdaad promotor. Hoewel je "niet zo'n jurk aan mag", vind ik het een eer die je geheel verdiend te beurt valt gezien je lange wetenschappelijke carrière en de manier waarop je je promovendi begeleidt. In het begin botste we soms (jij met je sanguinische, Rotterdamse inborst en ik met mijn soms [te] flegmatische reactie daarop), maar uiteindelijk konden we prima door één deur. Er zijn veel momenten die ik zal koesteren, van het 'even' neerploffen op één van je rode stoeltjes, de absurde roadtrip door de States met de familie, of de bijna vaderlijk bezorgde appjes de dagen na het finishen van de Ironman om wel voldoende te blijven drinken.

Geachte prof. dr. Delhaas, beste Tammo. Hoe vaak ben ik niet gefrustreerd aan de slag gegaan met het zout dat jij op mijn manuscriptelijke slakken hebt gelegd. Om je vervolgens toch wel weer gelijk te moeten geven als ik tevreden naar het verbeterde werk zat te kijken. Je bent een van de meest kritische mensen die ik ken en hebt mij zeker gevormd als wetenschapper. Het beiden met een gestrekt been een discussie aangaan was onderdeel van die vorming. Ondanks je (soms?) sterk uitgesproken mening, gaat het je er volgens mij niet zozeer om de ander te overtuigen van jouw gelijk, maar eerder de ander uit de tent te lokken en zelf een mening te vormen. Dank daarvoor.

Geachte dr.ir. Huberts, beste Wouter, de techneut van het stel. Allereerst dank voor alle tijd en energie die je hebt besteed om deze simpele dokter technisch en natuurkundig bij te spijkeren. Ik denk dat die kennis en vaardigheden mij een betere arts zullen maken. Dank daarvoor. Maar ook voor je benaderbare begeleiding. Met een kantoor naast elkaar was het natuurlijk makkelijk bij jou binnen te lopen, maar ik ging dan nooit weg zonder (soms wat uitvoerig) antwoord. Hoewel we daar beiden een handje van hebben, overigens. 
De leden van de beoordelingscommissie; prof. dr. J.P. Kooman (voorzitter), prof. dr. N.D. Bouvy, prof. dr. M.W. de Haan, prof. dr. ir. P. Verdonck en dr. A.S. Bode, wil ik hartelijk bedanken voor hun tijd en bereidheid om mijn proefschrift te beoordelen.

Dr. Van Loon, lieve Magda, bedankt voor alles! Het mij in het begin op sleeptouw nemen, het delen van je kennis en ervaring rondom shunts en dialysepatiënten, je inzet en inclusies voor de ShuntSimulatieStudie, noem het maar op. Vooral het koffiekwartiertje (of soms driekwartiertje) in de ochtend was altijd een goed begin van de dag. Mede door jou heb ik het erg naar mijn zin gehad de afgelopen vier jaar en ik ben dan ook heel blij dat je als paranimf aan mijn zijde staat tijdens mijn verdediging.

Collegae en oud-collegae van de Biomedical Engineering, bedankt dat ik jullie cliniclown mocht zijn. Maar ook voor de introductie in LaTeX en MATLAB - en het vele troubleshooting dat daarmee gepaard ging als ik bijvoorbeeld weer eens ronde haken in plaats accolades zat te gebruiken - en het verbreden van mijn horizon. Allereerst Tommy, samen ooit begonnen aan het 3S-project. Dank voor het aanleren van de basics van MATLAB (heuj! automatische brievengenerator), de gezelligheid op de kamer gedurende twee jaar tijdens jouw PDEng-opleiding en het begenadigd instarten van Joe Cocker. Claire, de mater familias en bijzonder gezellige buurvrouw. Het doet me deugd dat mijn foto nog steeds op je bureau staat, hopelijk maakt dit het afscheid dragelijker. Bedankt dat je het zootje ongeregeld in bedwang weet te houden. Peter, medeafdelingsclown en mede-oprichter van de BM2wielers. Dank voor de gezelligheid, grappen en grollen, en de vele mooie en aangename kilometers door het Limburgse en Belgische heuvelland. Andere leden van de BM2wielers; Maarten, Frank van R, Aurore, en Thijmen, dank ook voor jullie aanwezigheid en gezelligheid bij de nodige ritjes/ritten en pizzajacht bij terugkomst in Maastricht. Ben, een dikke middelvinger voor jou! Maar dan bedoeld als bedankje voor al je hulp als ik weer eens vastliep met MATLAB en voor je bijna dagelijkse gebuurt. You wink at me, I'll Wenkebach. Joost, tuupke, soms komt er een hoop onzin uit je (mooi!), maar met je serieuze kant was het lekker filosoferen over of reflecteren op academia. Koen, dank voor je levenslessen, je stimulering mij ook als onderwijzer verder te ontwikkelen en de met muziek omlijste etentjes. Lauren, douchebag Dupuis, dank voor de Fridays, shenanigans, en bitches. Pamir, kamergenoot, mits je niet druk was met alle experimenten voor jouw promotie. Veel succes met het voortzetten daarvan en dank voor de gezelligheid op de kamer en op de congrestripjes. Sjeng, de vaattoegang georiënteerde techneut. Jij in het bijzonder bedankt voor je $3 S$ hand-en-spandiensten en natuurlijk de congresongein. Arnold, Bart, Erik, Frank B, 
Jeroen, John, Marije, Myrthe, Nick, Raoul en Theo ook bedankt voor jullie bijdrage aan de fijne sfeer bij de BME.

Dames en heer van het vaatlab: Sandra, Evelien, Bianca, Jeroen, Renske en Ineke. Dank voor al het geduld om mij de fijne kneepjes van het duplexen uit te leggen. Dit maakte het implementeren van de duplexprotocollen in de andere ziekenhuizen makkelijker. Daarnaast bedankt voor jullie inzet voor de patiënteninclusie en follow-up en uiteraard ook de gezelligheid op het vaatlab als ik weer eens kwam buurten of op de HVC-borrels - gelukkig hebben we de foto's nog. Laura, jou wil ik bedanken voor je hulp bij poli- en duplexplanningen.

Het moge duidelijk zijn dat het pièce de résistance van dit proefschrift - de ShuntSimulatieStudie - zeker niet tot een goed einde gekomen zou zijn zonder de hulp van heel veel mensen: vaatchirurgen, vaatlaboranten, physician assistants, verpleegkundig specialisten, dialyseverpleegkundigen, onderzoekers, en met name de patiënten die geheel belangeloos mee hebben gedaan. ledereen persoonlijk bedanken voert te ver, maar een aantal mensen moeten expliciet bedankt worden. Per centrum (op volgorde van deelname) zijn dat (Principal Investigator voorop) de volgende mensen. Maasstad ziekenhuis, Rotterdam: André (dr. De Smet), Patricia, Liesbeth, Jeanne en Daniëlle. Flevoziekenhuis, Almere: Laurens (dr. Huisman), Karin, Mare, Margriet en Danny. Catharina ziekenhuis, Eindhoven: Philippe (dr. Cuypers), Nicole, Jenny en Stefanie. Laurentius ziekenhuis, Roermond: Felix (dr. Schlösser), Hendrik, Bas, Sabine en Monique. Slingeland ziekenhuis, Doetinchem: Susan (dr. Lemson) en Anita. St. Antonius ziekenhuis, Nieuwegein: Jan (dr. Wille), Stefan en Carolien. Zuyderland Medisch Centrum, Heerlen en Sittard: Lee (dr. Bouwman), Kees-Jan, Lars, Monica, Lilianne en Yvonne. UMC Utrecht: Raechel (dr. Toorop), Bart-Jeroen, Alferso, Shanna en Mourad.

Dan mag ik natuurlijk, naast Magda, de andere twee vaatdames niet vergeten. Carina, jij ook erg bedankt voor de gezelligheid bij het koffiekwartiertje aan het begin van de dag en dat ik je persoonlijke ICT-mannetje mocht zijn. Stella, jij daarnaast bedankt voor je inzet voor mijn steeds verdere inburgering cq. assimilatie in het Limburgse - get sjoen.

De Maastrichtse vaatchirurgen, heel erg bedankt dat ik de afgelopen vier jaar bij jullie mee heb mogen meelopen en back benchen bij de overdracht en vaatbesprekingen. Hierdoor werd ik er bij tijd en wijle weer aan herinnerd waarom ik toch achter die 
computer gekropen ben. Bovendien stond ik zo met minimaal nog een voorvoet in de kliniek. Prof. dr. Jacobs en prof. dr. Schurink, dank voor de inkijk bij de indrukwekkende aortachirurgie die jullie bedrijven. Drs. Van Laanen, beste Jorinde en dr. Peppelenbosch, beste Noud, jullie in het bijzonder bedankt voor jullie bijdrage aan de studie en gezelligheid op de trips naar Barcelona en Ljubljana. Dr. Daemen, beste Jan Willem, dank dat ik af en toe kon assisteren op OK. Dr. Mees, beste Barend, dank voor je inbreng bij de onderzoeksbesprekingen en dank dat je me bij het cabaret voor Chirurgendagen hebt betrokken. En vooral dr. Snoeijs, beste Maarten, bedankt dat je het voorzetten van de ShuntSimulatieStudie hebt gegarandeerd. $\mathrm{Na}$ al die jaren is het toch een beetje mijn kindje, welke in goede handen terecht gaat komen. Ik kijk uit naar onze verdere samenwerking. Letty bedankt dat jij het stokje over gaat nemen.

Toen was het na vier jaar computeren weer tijd om te gaan dokteren. En wat heb ik het naar mijn zin! Beste collegae van de Heelkunde Zuyderland, wat is het een genoegen met jullie te mogen samenwerken. Dr. Sosef en drs. De Loos, bedankt voor het gestelde vertrouwen. Mijn mede-assistenten ben ik bijzonder veel dank verschuldigd voor het zo soepeltjes laten verlopen van mijn 'terugkeer'. Jullie zijn een mooie club!

Maastrichtse Studenten Triathlon Vereniging Ferro Mosae. Dank voor de kennismaking met de inmiddels wat uit de klauwen gelopen hobby - of hobby's? Kunnen kiezen is klaarblijkelijk niét één van de disciplines. Van een blokkie om van 16k in de marge naar het finishen van de Ironman Maastricht in 2018. Gaandeweg bleek het een mooie uitlaatklep voor van alles en nog wat en dus een belangrijk onderdeel van mijn leven en het voltooien van dit boekje.

Ook hierbij zijn er wat mensen die persoonlijk bedankt moeten worden. Allereerst mijn medebestuursleden van het jaar '17-'18: Jasper, Loïc en Nick. Het was een genoegen samen met jullie een jaar voor de vereniging te mogen zorgen. Naast de mooie sportieve resultaten afgelopen jaar, denk ik dat we vooral onze stempel hebben gedrukt op de sociale kant van de vereniging. Daarom is het leuk te zien dat de Ferro Mondae dit jaar navolging heeft gekregen. Bestuur '18-'19 (Andreas, Eva, Remco, Audrey en Jeanine) heel veel succes en plezier. Roman, Thara en Thomas, als de wat vastere trainingsmaatjes, bedankt dat jullie de voorbereidingen op de Ironman dragelijker maakten.

Dudes! Dank voor de vele gezellige momenten door de jaren heen, de bierfestivalletjes her en der, de zelfgebrouwen biertjes, verscheidene Risk-veldslagen op campings, bij 
blokhutten of naast bergmeren, de kerstdiners, meermaals het Oktoberfest in München (of we in mei al wisten wat we in september wilden eten?) en noem het maar op. Maar ook duizendmaal dank voor de oprechte interesse in de inhoud en vooruitgang van mijn proefschrift. Het was af en toe fijn ventileren. Ilja en Thijs, ooit zijn we samen als middelbare schoolvrienden naar Maastricht vertrokken om te studeren. Hoewel ik daar nog steeds ben en jullie in Ede en Den Bosch wonen, is de vriendschap onverminderd hecht en de humor nog steeds kurkdroog. En dat waardeer ik. Stefan, medeMaastrichtenaar, nog zo'n droogkloot. Jij daarnaast bedankt voor je rol als statistiekvraagbaak. Tjibbe, wie had dit gedacht, al vrienden van voor de basisschool en nu een vermelding in een proefschrift. Jouw gedrevenheid is soms jaloersmakend, maar tegelijkertijd ook inspirerend. Succes met jouw promotie! Mike, hoewel onze paden iets anders gelopen zijn na B10 op het Mheenpark, is het toch iedere keer weer mooi elkaar te zien. Bedanken jullie dudes de Dudettes ook namens mij?

De \#WeZijnErBijna! Fanclub+1, Yannick, Marlies en Hester. Nu ik dit zit te schrijven realiseer ik me hoe toepasselijk \#WeZijnErBijna! Fanclub+1 is: altijd wel weer een nieuwe stip op ieders horizon. Hoewel het steeds lastiger wordt met $z^{\prime} n$ allen af te spreken nu we in Maastricht, Utrecht, Apeldoorn en Rotterdam zitten, zijn de WhatsApp live feeds van onder andere We Zijn Er Bijna, Nu We er Toch Zijn of Heel Holland Bakt mooie warmhoudertjes tot we weer eens samen zijn. (Dat het mogelijk ook een alarmsignaal is, al die burgerlijke MAX programma's, laat ik in het midden.) Maar als we dan weer eens samen zijn, is het net alsof we elkaar vorige week nog hebben gezien, zo vertrouwd is het. Voor nu vind ik het mooi dat onze promoties elkaar snel opvolgen en we deze momenten kunnen delen.

Lieve Tom, ik hoop dat je je paspoort meeneemt naar de verdediging, want er zullen er weer genoeg zijn die niet geloven dat die ene paranimf mijn broertje is. Ondanks dat we zowel qua uiterlijk als innerlijk ontzettend verschillen, ben ik heel gelukkig dat wij dit moment mogen delen. Zonder überhaupt te weten wat het inhield, had je al volmondig ja gezegd op mijn vraag om mijn paranimf te zijn. Ik houd van je en ben trots op je. Lieve Annemiek, schoonzus en daarmee aanwinst voor de familie. Je opgewektheid en enthousiasme werken aanstekelijk. Bedankt ook voor je oprechte interesse. Romy, mijn lieve, kleine nichtje. Deze trotse oom wil dat je, tegen de tijd dat je dit zelf kunt lezen, weet dat het een waar genot is om je te zien opgroeien. Ik kijk dan ook uit naar alle jaren die nog gaan volgen. En met die ouders van je wordt het sowieso iets moois. 
Lieve, lieve ouders. Vader en Moeder. Gerrit en Elly. Bedankt - en ik denk dat ik hier namens velen spreek - dat jullie mij op de wereld hebben gezet. Ik ben er blij mee en geniet er ontzettend van. Maar ik wil jullie vooral heel erg bedanken voor hoe jullie zijn als ouders en voor alle kansen die jullie mij geboden hebben. En wat er ook is, ik weet dat ik bij jullie terecht kan. Hoewel de fysieke afstand onverminderd groot is, heb ik het idee dat we de laatste jaren steeds dichter bij elkaar komen. lets met volwassen worden? Ik houd van jullie en prijs mezelf gelukkig met zulke ouders.

Liefste Natascha; es iech diech zeen bin iech blij tot iech leef, iech bin zoe verleef. Vergeef me de copy-pasta van Beppie: ik ben nog niet zo ver in mijn Taolkelender vaan 't Mestreechs. Dus nog even gewoon in het Nederlands: wat ben ik blij dat je in mijn leven gekomen bent. Je maakt het zoveel rijker en mooier. Bedankt voor alle ruimte en steun die je me geeft om mijn sportieve en professionele dromen na te jagen. Ik houd van je. lech haw vaan diech.

Disclaimer:

De auteur heeft zijn uiterste best gedaan om de rechthebbenden op een bedankje te achterhalen, noemen en daadwerkelijk te bedanken. Meent $u$ rechthebbende te zijn van één of meer bedankjes die onverhoopt niet gemaakt zijn, dan wordt u bij dezen ook bedankt. Bedankt *

*) schrijf hier desgewenst met blauwe pen uw eigen naam. 
DANKWOORD | 219 

222 | APPENDIX 4 


\section{BIOGRAPHY}

Niek Zonnebeld was born on February $3^{\text {rd }}, 1989$ in Apeldoorn, The Netherlands. In 2007 he graduated from Veluws College Walterbosch in Apeldoorn. After studying Psychology at Maastricht University for one year, he started his Medical studies at the same university in 2008. During his Bachelors he started working as a Kidney Racer at the Bureau Donatie- en Transplantatiecoördinatie (BDTC) of the

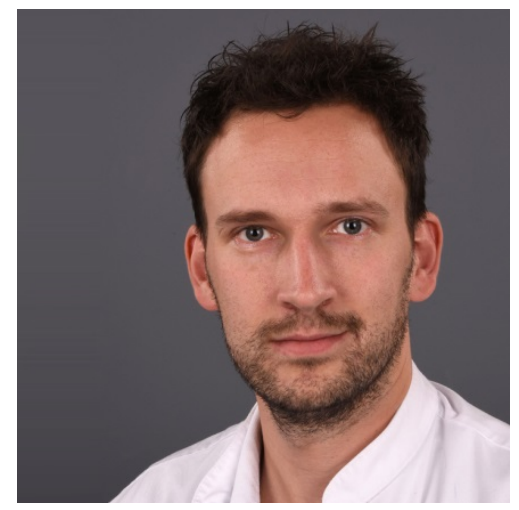
Maastricht University Medical Center (UMC) under supervision of prof. dr. L.W.E. van Heurn. This job sparked his interest in surgery, and kidney transplantations in particular. His clinical internships included Ophthalmology in Pretoria, South Africa, Traumatology at the Isala hospital in Zwolle (supervised by dr. S.H. van Helden), and for his senior clinical and scientifical internship Vascular and Transplantation surgery he returned to the BDTC and the Department of Surgery of the Maastricht UMC (supervised by prof. dr. L.W.E. Van Heurn). The scientifical internship sparked his academic aspirations, which resulted in a desire to attain his $\mathrm{PhD}$.

Immediately after graduation in September 2014, Niek started his PhD research at the department of Biomedical Engineering at Maastricht University under supervision of dr. J.H.M. Tordoir, prof. dr. T. Delhaas, and dr. ir. W. Huberts. The present dissertation is the result of this $\mathrm{PhD}$ research. Although intellectually challenging, physically it was not demanding and Niek therefore started training for triathlons with the student triathlon association Ferro Mosae. In 2018, the same year he was president of Ferro Mosae, he finished the Ironman of Maastricht.

In October 2018 he started working as a surgical resident not in training at the Department of Surgery at the Zuyderland Medical Center (supervised by dr. M.N. Sosef and drs. E.R. de Loos), where he still works. 


\section{PUBLICATIONS}

Zonnebeld N, Huberts W, van Loon MM, Delhaas T, Tordoir JHM. Preoperative computer simulation for planning of vascular access surgery in hemodialysis patients. J Vasc Access. 2017;18(Suppl. 1):118-24.

Zonnebeld N, Maas TMG, Huberts W, van Loon MM, Delhaas T, Tordoir JHM. Preoperative Duplex Ultrasonography in Arteriovenous Fistula Creation: Intra- and Interobserver Agreement. Eur J Vasc Endovasc Surg. 2017;54(5):613-9.

Tordoir JHM, Zonnebeld N, van Loon MM, Gallieni M, Hollenbeck M. Surgical and Endovascular Intervention for Dialysis Access Maturation Failure During and After Arteriovenous Fistula Surgery: Review of the Evidence. Eur J Vasc Endovasc Surg. 2018;55(2):240-8.

Zonnebeld N, Huberts W, Tordoir JHM, Delhaas T. Modeling Strategies in Arteriovenous Fistula Maturation and Functioning: Accomplishments and Improvements. In preparation.

Zonnebeld N, Huberts W, van Loon MM, Delhaas T, Tordoir JHM. Natural vascular remodeling after arteriovenous fistula creation in dialysis patients with and without previous ipsilateral vascular access. Under review.

Zonnebeld N, Tordoir JHM, van Loon MM, de Smet AAEA, Huisman LC, Cuypers PWM, Schlösser FJV, Lemson MS, Heinen SGH, Bouwman LH, Toorop RJ, Huberts W, Delhaas T, on behalf of the Shunt Simulation Study group. Preoperative patient-specific flow predictions to improve hemodialysis arteriovenous fistula maturation (Shunt Simulation Study): a randomized controlled trial. Under review.

\section{Not included in this thesis}

Huberts W, Heinen SGH, Zonnebeld N, van den Heuvel DAF, de Vries J-PPM, Tordoir JHM, Hose DR, Delhaas T, van de Vosse FN. What is needed to make cardiovascular models suitable for clinical decision support? A viewpoint paper. Journal of Computational Science. 2018;24:68-84.

van Oevelen M, Abrahams AC, Weijmer MC, Nagtegaal T, Dekker FW, Rotmans Jl, Meijvis SCA; on behalf of the DUCATHO study group. Precurved non-tunnelled catheters for haemodialysis are comparable in terms of infections and malfunction as compared to tunnelled catheters: A retrospective cohort study. J Vasc Access. 2018. 


\section{PRESENTATIONS}

\section{Invited}

Max Taks Vaatsymposium 2017

Doetinchem, The Netherlands

Hulp in de arm genomen: computersimulaties voor shuntaanleg

Dialysis Initiatives Nefrologen 2017

Doorn, The Netherlands

De ShuntSimulatieStudie

European Vascular Course 2017

Maastricht, The Netherlands

ShuntSimulatieStudie (3S): Computer simulatie voor de aanleg van fistels

\section{Oral presentations}

9th Congress of the Vascular Access Society 2015

Barcelona, Spain

10th Congress of the Vascular Access Society 2017

Ljubljana, Slovenia (2 presentations)

Vaatdagen 2017

Noordwijkerhout, The Netherlands

XVI Hemodialysis Symposium of the Vascular Access Society of the Americas 2018

New Orleans, The United States of America

\section{Poster presentations}

Pélerin Wetenschapssymposium 2016

Maastricht, The Netherlands

Symposium Experimenteel Onderzoek Heelkundige Specialismen 2016

Utrecht, The Netherlands

European Society for Vascular Surgery Springmeeting 2017

Leiden, The Netherlands

10th Congress of the Vascular Access Society 2017

Ljubljana, Slovenia

XVI Hemodialysis Symposium of the Vascular Access Society of the Americas 2018

New Orleans, The United States of America (3 posters) 
AWARDS

\section{ADDITIONAL ACTIVITIES}

$\begin{array}{ll}2017 \text { - } 2018 & \text { President of the Maastricht student triathlon association Ferro Mosae } \\ 2018 \text { - } & \text { Reviewer for the Journal of Vascular Access } \\ 2018 \text { - } & \text { Reviewer for the European Journal of Vascular and Endovascular Surgery } \\ 2018 \text { - } & \begin{array}{l}\text { Member Raad Kwaliteit en Veiligheid, Zuyderland Medical Center, } \\ \text { Heerlen/Sittard, The Netherlands }\end{array}\end{array}$




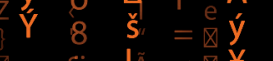

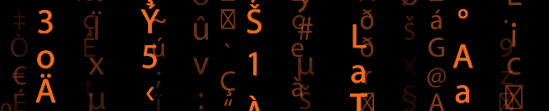

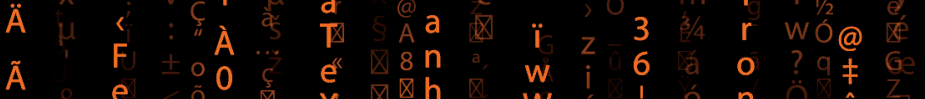

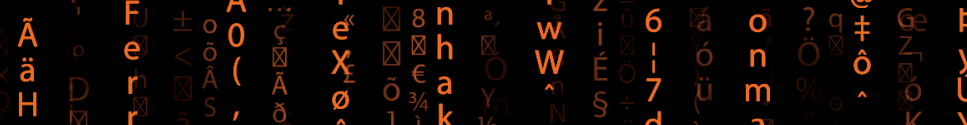

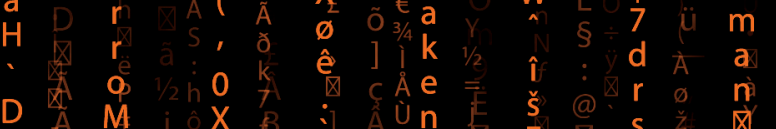

0 A

é $1 / 2$ है

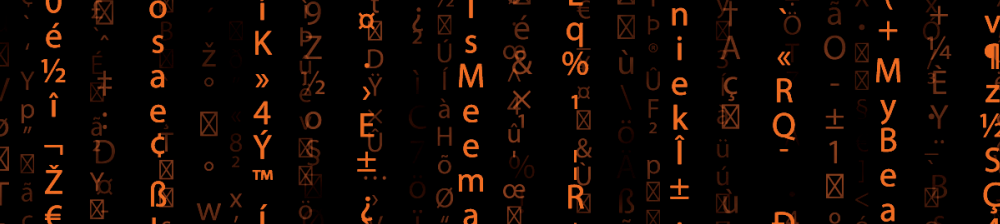

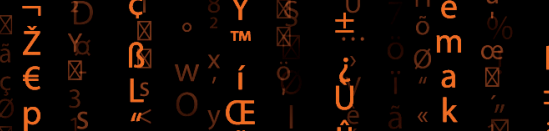

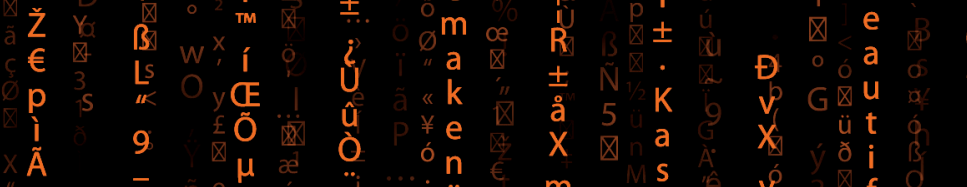

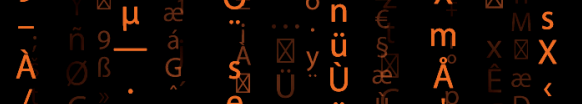

$\varnothing$

Y.

ê. $\triangle A$ a

$\wedge$

ï

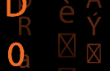

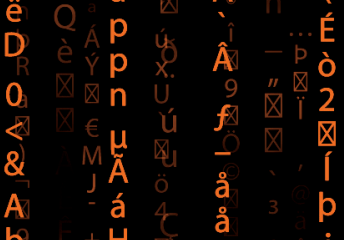

,

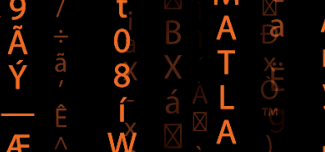

$\stackrel{A}{\mathbb{C}}$

$\underset{\mathrm{F}}{ }$

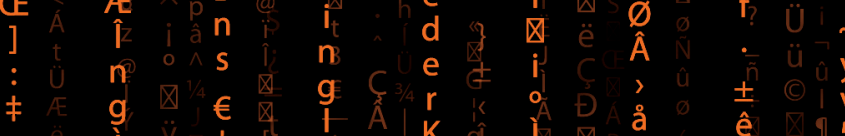

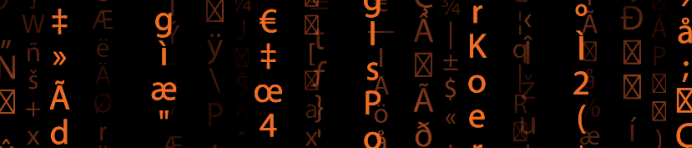

A

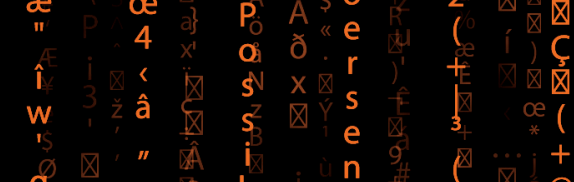

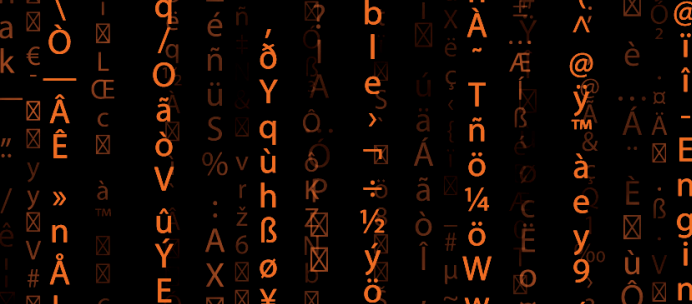

$\mathbb{Q}$
$V$
$\mathrm{~V}$

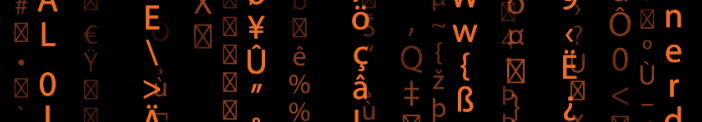

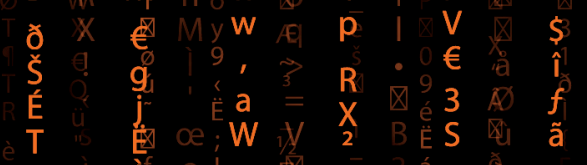
u w

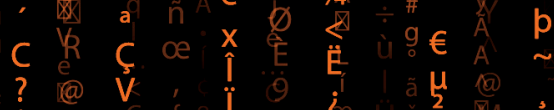
U ì $\begin{array}{ll}\text { aे } & \emptyset \\ \text { i } & 1 / 2\end{array}$ l 0 . 4 ठ० 4

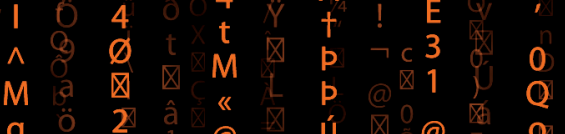
q $1 / 20$ $S \stackrel{T}{T}$

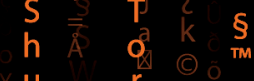

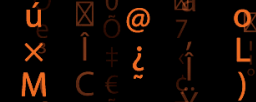

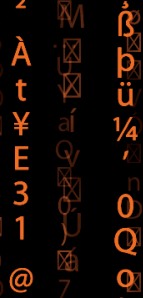
区 $€$ U $\mathrm{X}$ 冈A A

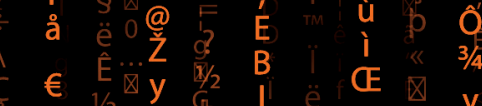

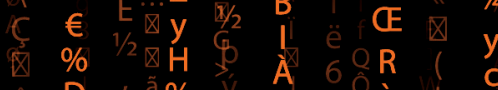
a D $\mathrm{D}$ \% \% y

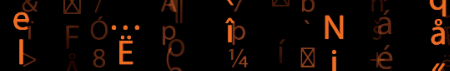

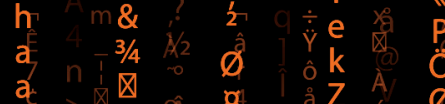

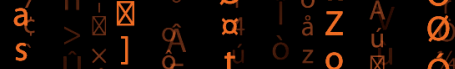

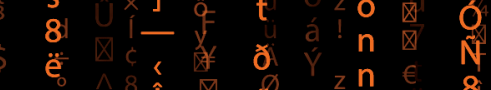
$\pm$ 